SLAC -348

$\mathrm{UC}-34 \mathrm{D}$

$(\mathrm{T} / \mathrm{E})$

\title{
PRODUCTION AND DECAY OF HEAVY TOP QUARKS
}

\author{
Russel P. Kaufman \\ Stanford Linear Accelerator Center \\ Stanford University \\ Stanford, California 94309
}

August 1989

Prepared for the Department of Energy

under contract number DE-AC03-76SF00515

Printed in the United States of America. Available from the National Technical Information Service, U.S. Department of Commerce, 5285 Port Royal Road, Springfield, Virginia 22161. Price: Printed Copy A05, Microfiche A01.

* Ph. D. thesis 


\section{PRODUCTION AND DECAY OF HEAVY TOP QUARKS}

Russel P. Kauffman, Ph.D.

Stanford University, 1989

Experimental evidence indicates that the top quark exists and has a mass between 50 and $200 \mathrm{GeV} / \mathrm{c}^{2}$. The decays of a top quark with a mass in this range are studied with emphasis placed on the mass region near the threshold for production of real $W$ bosons. Topics discussed are: 1) possible enhancement of strange quark production when $M_{W}+m_{s}<m_{t}<M_{W}+m_{b}$; 2) exclusive decays of $T$ mesons to $B$ and $B^{*}$ mesons using the non-relativistic quark model; 3) polarization of intermediate $W$ 's in top quark decay as a source of information on the top quark mass.

The production of heavy top quarks in an $e^{+} e^{-}$collider with a center-of-mass energy of $2 \mathrm{TeV}$ is studied. The effective-boson approximation for photons, $Z^{0}$ 's and $W$ 's is reviewed and an analogous approximation for interference between photons and $Z^{0}$ 's is developed. The cross sections for top quark pair production from photon-photon, photon- $Z^{0}, Z^{0} Z^{0}$, and $W^{+} W^{-}$fusion are calculated using the effective-boson approximation. Production of top quarks along with anti-bottom quarks via $\gamma W^{+}$and $Z^{0} W^{+}$fusion is studied. An exact calculation of $\gamma e^{+} \rightarrow \bar{\nu} t \bar{b}$ is made and compared with the effective- $W$ approximation. 


\section{ACKNOWLEDGMENTS}

Since no project is complete until the acknowledgement is done I would like to express my gratitude to the people who have contributed to me and to this work:

To my advisor, Fred Gilman, for his calm guidance over the last five years;

To the members of my Reading Committee: Dick Blankenbecler, Stan Brodsky, and Savas Dimopoulos, for their careful reading of the manuscript;

To Professor Peter Ray from Biology, for chairing an oral far outside his field of expertise;

To my colleagues in the SLAC theory group: Claudio Dib, Eran Yehudai, and Yosef Nir, for many illuminating discussions; and Kent Hornbostel, Paul Griffin and Chris Wendt for making the Central Lab third floor the cool, hip, happenin' place that it is;

To Sharon Jensen and Robbin Nixon, for endless $\mathrm{T}_{\mathrm{E}} \mathrm{X}$ questions, countless phone messages and the bottomless coffee pot;

To Kevin Johnson and the rest of the crew in Technical Illustrations for their work on the figures;

To all the members of Dirk's Jerks Athletic Association, past and present, but especially Jordan Nash, Jim Mactiernan, Wayne Rigby, and Tom Duffy, because there is a Jerk in all of us just waiting to get out;

To my wife, Gina, for her love and support;

To my parents, Delbert and Helen, for everything. 


\section{TABLE OF CONTENTS}

1. Introduction . . . . . . . . . . . . . . 1

2. Top Quark Decays when $m_{t} \simeq M_{W}+m_{b}$. . . . . . . . . 4

2.1 Introduction . . . . . . . . . . . . . . . . 4

2.2 The Decay Rate for $t \rightarrow b e^{+} \nu_{e} \ldots \ldots . . . . . .44$

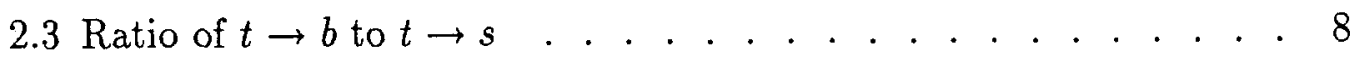

2.4 Exclusive Modes . . . . . . . . . . . . . . . . . . . 10

$2.5 W$ Polarization in $t$ Decay . . . . . . . . . . . . . 15

3. Top Quark Production in $e^{+} e^{-}$Colliders . . . . . . . . . . 21

3.1 Introduction . . . . . . . . . . . . . . 21

3.2 The Effective-Photon Approximation . . . . . . . . . 23

3.3 Two-Photon Production of Top Quarks . . . . . . . . . . 28

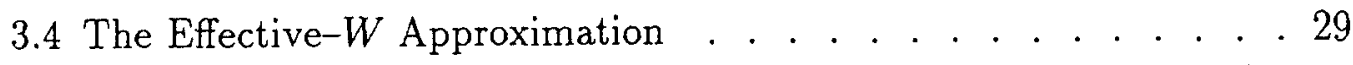

3.5 Top-Quark Production from $W W$ Fusion . . . . . . . . . . . 41

3.6 Top-Quark Production from $Z Z$ Fusion . . . . . . . . . . . . 50

3.7 Photon $-Z^{0}$ Fusion Production of Top Quarks . . . . . . . . 55

3.8 Photon- $Z^{0}$ Interference . . . . . . . . . . . . 57

3.9 Production of $t \bar{b}$ Pairs from $\gamma W$ Fusion . . . . . . . . 66

3.10 Production of $t \bar{b}$ Pairs from $W Z$ Fusion $\ldots \ldots . . . . .73$ 
3.11 Beamstrahlung . . . . . . . . . . . . . . . 78

3.12 Summary and Conclusions . . . . . . . . . . . . . 81

References .................. . . . 83 


\section{LIST OF FIGURES}

2.1 Feynman diagram for the semi-leptonic decay of the top quark . . . . . . . . . . . . . . . . . . . . . 4

2.2 Semi-leptonic decay rate of the top quark . . . . . . . . . . . 7

2.3 Differential semi-leptonic decay rate, $d \Gamma\left(t \rightarrow b e^{+} \nu_{e}\right) / d Q^{2}$. . . . . . . . . . . . . . . . . . . . . . . 8

2.4 Ratio of $t \rightarrow s$ to $t \rightarrow s$ with Kobayashi-Maskawa factors removed . . . . . . . . . . . . . . . . . . . . 9

2.5 Ratio of longitudinal to transverse widths. . . . . . . . . . 18

2.6 Ratio of the difference of the transverse rates to the sum . . . . . 20

3.1 The Feynman diagrams for $e^{+} e^{-} \rightarrow \gamma, Z^{0} \rightarrow t \bar{t} \quad . \quad$. . . . . . . . 22

3.2 One-photon production of an arbitrary final state $X$. . . . . . . . 24

3.3 Flux of photons in the electron . . . . . . . . . . . . . 27

3.4 The Feynman diagrams for $\gamma \gamma \rightarrow t \bar{t}$. . . . . . . . . . . . . . . . 28

3.5 Cross section for two-photon production of top quarks . . . . . . . 29

3.6 The production of an arbitrary final state $\mathrm{X}$ via exchange of a massive vector boson $V$. . . . . . . . . . . . . . 31

3.7 Fluxes of $W$ bosons in the electron in leading logarithmic approximation . . . . . . . . . . . . . . . . . . . . . 39

3.8 Exact fluxes of $W$ bosons in the electron . . . . . . . . . . . . 40 
3.9 Production of top quarks via $W W$ fusion . . . . . . . . . . . . . 41

3.10 Peripheral diagram neglected in the effective- $W$

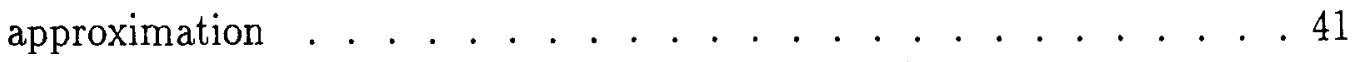

3.11 Feynman diagrams for $W^{+} W^{-} \rightarrow t \bar{t}$. . . . . . . . . . . . . . . 42

3.12 Contributions to top quark production from fusion of transverse $W^{\prime}$ 's . . . . . . . . . . . . . . . . . . . . . . . 45

3.13 Contributions to top quark production from fusion of one transverse $W$ with one longitudinal $W$. . . . . . . . . . . 48

3.14 Contributions to top quark production from fusion of longitudinal $W$ 's . . . . . . . . . . . . . . . . . . . . 49

3.15 Full cross section for top quark production from $W W$ fusion .. . . . . . . . . . . . . . . . . 50

3.16 Feynman diagrams for $Z^{0} Z^{0} \rightarrow t \bar{t}$. . . . . . . . . . . . . . . . 51

3.17 Contributions to top quark production from fusion of two tranverse $Z^{0}$ 's and from one transverse $Z^{0}$ with one longitudinal $Z^{0}$. . . . . . . . . . . . . . . . . . . . 53

3.18 Contributions to top quark production from fusion of two longitudinal $Z^{0}$ 's. . . . . . . . . . . . . . . . . . . 54

3.19 Full cross section for top quark production from

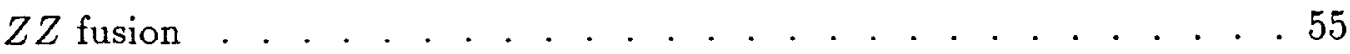

3.20 Feynman diagrams for $\gamma Z^{0} \rightarrow t \bar{t}$. . . . . . . . . . . . . . . . . 56 
3.21 Contributions to top quark production from $\gamma Z^{0}$ fusion . . . . . . . . . . . . 57

3.22 One-photon production and one- $Z^{0}$ production of an arbitrary final state $X \ldots \ldots \ldots$

3.23 Distributions for $\gamma Z^{0}$ interference . . . . . . . . . . 661

3.24 Diagrams contributing to $e^{+} e^{-} \rightarrow e^{+} e^{-} t \bar{t} \ldots \ldots 62$

3.25 Diagrams for terms involving three photons and one $Z^{0} \ldots$. . . . 63

3.26 Diagrams for terms involving two photons and two $Z^{0}$ s $\ldots . . .64$

3.27 Contributions to top quark production from $\gamma Z^{0}$ interference . . . . . . . . . . . . 66 66

3.28 Feynman diagrams for $\gamma W^{+} \rightarrow t \bar{b} \ldots \ldots 7$

3.29 Cross section for $t-\bar{b}$ production via $\gamma W$ fusion . . . . . . 70

3.30 Feynman diagrams for $e^{+} \gamma \rightarrow \bar{\nu} t \bar{b} \quad \ldots \ldots . \ldots 71$

3.31 Differential cross section $d \sigma\left(e^{+} \gamma \rightarrow \bar{\nu} t \bar{b}\right) / d x \ldots . . . . . .72$

3.32 Exact cross section for $t-\bar{b}$ production compared to the effective- $W$ result . . . . . . . . . . . . . . . 73

3.33 Feynman diagrams for $Z^{0} W^{+} \rightarrow t \bar{b} \ldots \ldots . . \ldots 74$

3.34 Contributions to $t \bar{b}$ production from $W Z$ fusion $\ldots . . . . .77$

3.35 Differential photon-photon luminosity from beamstrahlung . . . . . . . . . . . . . . . . 79 
3.36 Differential photon-electron luminosity from beamstrahlung . . . . . . . . . . . . . . . . . 79

3.37 Cross section for production of top quarks from fusion of beamstrahlung photons . . . . . . . . . . 80

3.38 Cross section for $t \bar{b}$ production by fusion of beamstrahlung photons with virtual $W$ bosons. . . . . . . . . . . . . . 81 


\section{Introduction}

The last character (except the Higgs) to be added to the cast of the Standard Model with three generations is the top quark. Since the top quark has yet to be discovered, the first question we must address is: "What top quark?" By the top quark we mean the partner to the $b$ quark in an $S U(2)$ doublet. The existence of the top quark is inferred from the measured properties of the $b$. Experiments at PEP and PETRA, in which $e^{+} e^{-} \rightarrow \gamma, Z^{0} \rightarrow b \bar{b}$ is measured, show a nonzero forward-backward asymmetry. This indicates that the axial coupling of the bottom to the $Z^{0}$ is non-zero, ruling out the possibility that the bottom quark is an $S U(2)$ singlet. Furthermore, if the $b$ were an $S U(2)$ singlet there would be decays mediated by flavor-changing neutral currents, such as $b \rightarrow s e^{+} e^{-}$, which are not seen. ${ }^{3}$ Lastly, the top quark is needed on theoretical grounds, in order that the Standard Model be anomaly-free.

The $t$ quark mass is constrained to be above $29 \mathrm{GeV}$ from TRISTAN, above 44 $\mathrm{GeV}$ from $\mathrm{UA} 1^{5}$, and above about $50 \mathrm{GeV}$ from theoretical considerations ${ }^{6}$ based on the ARGUS result ${ }^{7}$ for $B-\bar{B}$ mixing. In fact, the $B-\bar{B}$ mixing results, intcrpretcd within the standard model, would have one entertain $t$ quark masses in the vicinity of $100 \mathrm{GeV}$. Recent results ${ }^{8}$ from CDF, UA1, and UA2 show no evidence for a top quark with mass less than $60 \mathrm{GeV}$ and new data accumulated by CDF should be able to set a bound that approaches the $W$ mass.

In Chapter 2 we consider in some detail the transition region between the production of "virtual" and "real" $W$ 's in $t$ decays, i.e., values of $m_{t} \approx M_{W}+m_{b}$. The absolute width for a $t$ quark with a mass in this range has been considered 
previously, ${ }^{10}$ usually as a special case of a generic heavy quark decaying to a "real" $W$.

After reviewing some of this analysis at the beginning of Chapter 2, we examine some particular properties of the region where $m_{t} \approx M_{W}+m_{b}$, noting especially how the possibility of a sharp transition or threshold is smeared out by the finite width of the $W$. In Section 2.3, we consider the decay rate for $t \rightarrow s+W$ compared to that for $t \rightarrow b+W$. The first process is suppressed relative to the second by the ratio of Kobayashi-Maskawa matrix elements squared, $\left|V_{t s}\right|^{2} /\left|V_{t b}\right|^{2}$, which is known $^{11}$ to be $\approx 1 / 500$. There is a region, however, where the first process is above threshold for production of a real $W$, while the second is below threshold. The question of whether this can compensate for the Kobayashi-Maskawa suppression is answered (negatively) in Section 2.3.

In Section 2.4 we consider the possibility that the hadronic final state recoiling against the $W$ and containing a $b$ quark will be dominated by a very few hadronic states, rather than be a sum of many states in the form of a jet. We calculate the specific matrix elements in this case in the quark model-one of the few cases in which the nonrelativistic quark model may really be well-justified a priori.

This ties into Section 2.5, where we examine the relative population of longitudinal and transverse $W$ 's as we move through the threshold region. The ratio of decay widths involving longitudinal and transverse $W$ 's varies fairly rapidly near the threshold and we show how the associated lepton or quark jet angular distribution in the $W$ decay can be used to measure this quantity and help determine the $t$ quark mass to a few $\mathrm{GeV}$. 
Section 2.6 contains a summary of Chapter 2 and conclusions.

Chapter 3 is devoted to the study of top quark production via vector-boson fusion in an $e^{+} e^{-}$collider with a center-of-mass energy of $2 \mathrm{TeV}$. We first review the effective-photon approximation and then use it to calculate the production of $t-\bar{t}$ pairs via photon-photon fusion. We then review the effective-boson approximation for $W^{ \pm}$and $Z^{0}$ bosons, deriving a consistent set of distributions and showing that the interference terms between different helicities do not contribute.

In Sections 3.5-3.7 we discuss the production of $t-\bar{t}$ pairs via $W^{+} W^{-}, \gamma Z^{0}$, and $Z^{0} Z^{0}$ fusion. These calculations are carried out in the effective-boson approximation. We present the results as a function of $m_{t}$ for a variety of Higgs Inasses.

The analogue of the effective-boson approximation for the interference between photons and $Z^{0}$ 's is derived in Section 3.8. We then use this formalism to calculate the contribution of these interference terms to $e^{+} e^{-} \rightarrow e^{+} e^{-} t \bar{t}$.

The next two sections concern the production of top quarks with associated anti-bottom quarks. These processes proceed through $\gamma W^{+}$and $Z^{0} W^{+}$fusion. We calculate the cross section for $\gamma W^{+}$fusion in the effective- $W$ approximation and compare the result to an exact calculation of $e^{+} \gamma \rightarrow \bar{\nu} t \bar{b}$.

The luminosities for beamstrahlung photons are presented in Section 3.11. The cross sections for fusion of beamstrahlung photons into top quarks and interactions of beamstrahlung photons with positrons to produce $t-\bar{b}$ pairs are calculated.

We conclude Chapter 3 with a summary and comparison to previous results. 


\section{Top Quark Decays when $m_{t} \approx M_{W}+m_{b}$}

\subsection{INTRODUCTION}

The decays of a heavy top quark have a much different character than the decays of the lighter quarks. Even for values of $m_{t} \approx 50 \mathrm{GeV}$, the finite mass of the $W$ results in $a \approx 25 \%$ increase in the $t$ decay width over the value calculated with the point (infinite $M_{W}$ ) Fermi interaction; for $M_{t} \approx 100 \mathrm{GeV}$ we have decay into a "real" $W$ resonance and the width is proportional to $G_{F}$ rather than $G_{F}^{2}$. In this chapter we focus on the transition region between the production of "virtual" and "real" $W$ 's in $t$ decays, i.e., values of $m_{t} \approx M_{W}+m_{b}$.

\subsection{ThE DECAY RATE FOR $t \rightarrow b e^{+} \nu_{e}$}

Consider the semileptonic decay of $t$ to $b$. The tree-level width, for any value of $m_{t}$, can be calculated from the diagram in Figure 2.1 .

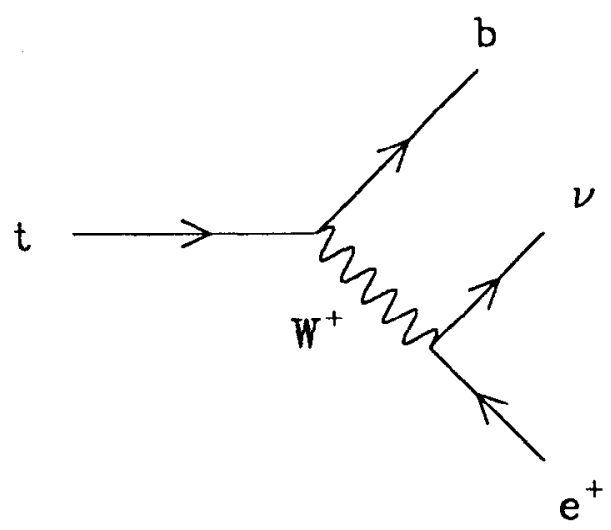

Figure 2.1 The Feynman diagram for the semi-leptonic decay of the top quark, $t \rightarrow b e^{+} \nu_{e}$. 


$$
\begin{aligned}
& \Gamma\left(t \rightarrow b e^{+} \nu_{e}\right)= \\
& \frac{G_{F}^{2} m_{t}^{5}}{24 \pi^{3}} \int_{0}^{\left(m_{t}-m_{b}\right)^{2}} d Q^{2} \frac{M_{W}^{4}|\mathbf{Q}|}{\left(Q^{2}-M_{W}^{2}\right)^{2}+M_{W}^{2} \Gamma_{W}^{2}}\left[2|\mathbf{Q}|^{2}+3 Q^{2}\left(1-\frac{Q_{0}}{m_{t}}\right)\right],
\end{aligned}
$$

where $\Gamma_{W}$ is the total width of the $W$ and the integration variable $Q^{2}$ is the square of the four-momentum which it carries, with the associated quantities

$$
\begin{aligned}
Q_{0} & =\left(m_{t}^{2}+Q^{2}-m_{b}^{2}\right) / 2 m_{t}, \\
|\mathrm{Q}|^{2} & =Q_{0}^{2}-Q^{2} .
\end{aligned}
$$

In general, the right-hand side of Eq. (2.1) should contain the square of the relevant Kobayashi-Maskawa matrix element, $\left|V_{t b}\right|^{2}$, which in the case of three generations is one to high accuracy.

In the limit that $m_{t} \ll M_{W}$, the momentum dependence of the $W$ propagator can be neglected and the expression simplifies to

$$
\begin{aligned}
\Gamma\left(t \rightarrow b e^{+} \nu_{e}\right) & =\frac{G_{F}^{2} m_{t}^{5}}{24 \pi^{3}} \int_{0}^{\left(m_{t}-m_{b}\right)^{2}} d Q^{2}|\mathbf{Q}|\left[2|\mathbf{Q}|^{2}+3 Q^{2}\left(1-Q_{0} / m_{t}\right)\right] \\
& =\frac{G_{F}^{2} m_{t}^{5}}{6 \pi^{3}} \int_{0}^{\left(m_{t}-m_{b}\right)^{2}} d Q^{2}|\mathbf{Q}|^{3} \\
& =\frac{G_{F}^{2} m_{t}^{5}}{192 \pi^{3}}\left[1-8 \Delta^{2}+8 \Delta^{6}-\Delta^{8}-24 \Delta^{4} \ln \Delta\right]
\end{aligned}
$$

where $\Delta=m_{b} / m_{t}$.

In the other limit, where $m_{t}$ is sufficiently above $M_{W}$, we may integrate over 
the Breit-Wigner for producing a "real" $W$, and using

$$
\Gamma\left(W^{+} \rightarrow e^{+} \nu_{e}\right)=\frac{G_{F} M_{W}^{3}}{6 \pi \sqrt{2}}
$$

rewrite Eq. (2.1) as

$$
\Gamma\left(t \rightarrow b+W \rightarrow b e^{+} \nu_{e}\right)=B\left(W \rightarrow e \nu_{e}\right) \cdot \frac{G_{F}|\mathbf{Q}|}{2 \pi \sqrt{2}}\left[2|\mathbf{Q}|^{2}+3 M_{W}^{2}\left(1-\frac{Q_{0}}{m_{t}}\right)\right],
$$

where now $Q^{2}=M_{W}^{2}$, so that $Q_{0}=\left(m_{t}^{2}+M_{W}^{2}-m_{b}^{2}\right) / 2 m_{t}$ and $|\mathbf{Q}|^{2}=Q_{0}^{2}-M_{W}^{2}$. For very large values of $m_{t}$, the width in Eq. (2.5) behaves as

$$
\Gamma\left(t \rightarrow b+W \rightarrow b e^{+} \nu_{e}\right)=B\left(W \rightarrow e \nu_{e}\right) \cdot G_{F} m_{t}^{3} / 8 \pi \sqrt{2},
$$

to be contrasted with Eq. (2.3).

The finite width of the $W$ determines the behavior of the rate as we cross the threshold for producing a real $W$. Once we are several full widths of the $W$ above threshold, the much larger width given in Eq. (2.5) for producing a "real" $W$ dominates the total $t$ decay rate. This is seen in Figure 2.2, where the $t \rightarrow b e^{+} \nu_{e}$ decay rate is plotted versus $m_{t}$. The dashed curve is the result in Eq. (2.5) which would hold for production of a real, infinitely narrow $W$, while the solid curve gives the result of integrating Eq. (2.1) numerically. ${ }^{12}$ For smaller values of $m_{t}$ the width is less than $G_{F}^{2} m_{t}^{5} / 192 \pi^{3}$ because of the finite value of $m_{b}$ [here taken to be $5 \mathrm{GeV}$, see Eq. (2.3)], but then is enhanced by the $W$ propagator as $m_{t}$ increases. The exact result quickly matches that for an infinitely narrow $W$ once we are several $W$ widths above threshold. The finite $W$ width simply provides a 


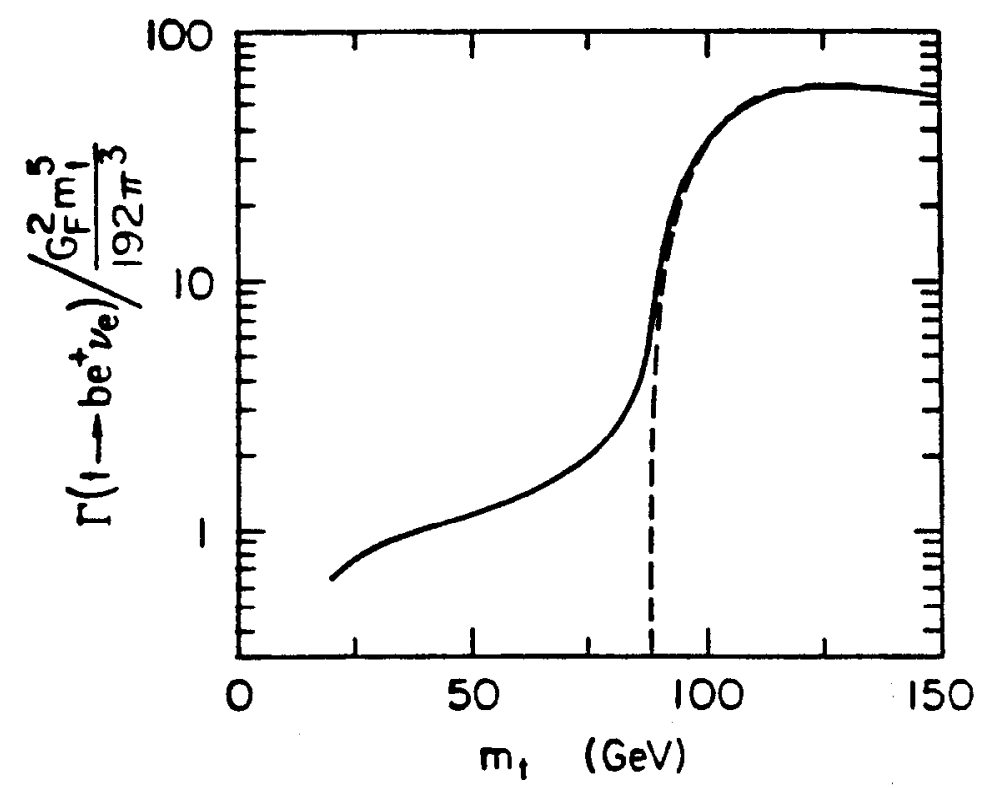

Figure 2.2 $\Gamma\left(t \rightarrow b e^{+} \nu_{e}\right) /\left(G_{F}^{2} m_{t}^{5} / 192 \pi^{3}\right)$ as a function of $m_{t}$ from the full expression in Eq. (2.1) for $M_{w}=83 \mathrm{GeV}, \Gamma_{w}=2.25 \mathrm{GeV}$ and $m_{b}=5 \mathrm{GeV}$ (solid curve), and from Eq. (2.5) for decay into a real, infinitely narrow $W$ (dashed curve).

smooth interpolation as the decay rate jumps by over an order of magnitude in crossing the threshold.

The peaking of the differential rate around the $W$-pole can be seen in Figure 2.3 , in which we plot $d \Gamma / d Q^{2}$ for a range of values for $m_{t}$. We see that for top masses above the threshold for real $W$ production the peaking of the distribution becomes pronounced and the bulk of the rate comes from values of $Q^{2}$ very near $M_{W}^{2}$. This rapid change in both the absolute rate and its phase-space distribution is what will drive the processes which we will study in the following sections. 


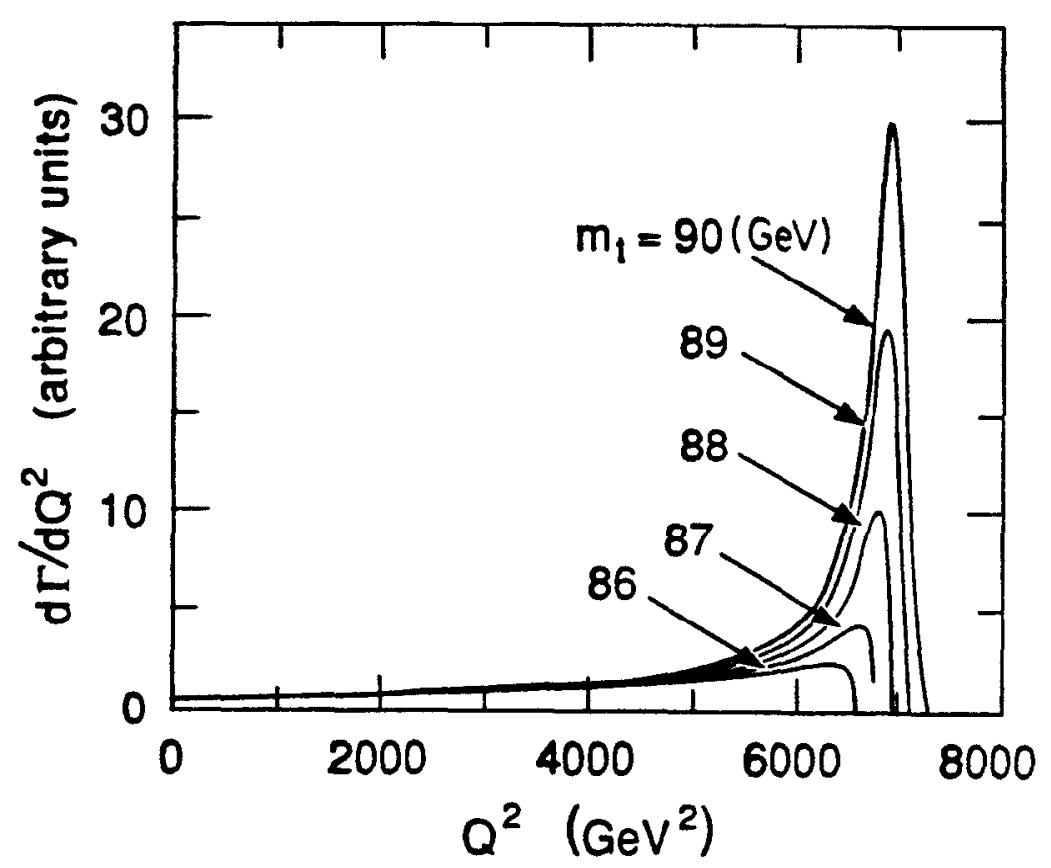

Figure 2.3 The differential width $d \Gamma\left(t \rightarrow b e^{+} \nu_{e}\right) / d Q^{2}$ (in arbitrary units), as a function of $Q^{2}$ for a succession of top quark masses, spanning the threshold for decay into a real $W$ and $b$ quark. The masses are taken as $M_{w}=83 \mathrm{GeV}$ and $m_{b}=5 \mathrm{GeV}$.

\subsection{RATIO OF $t \rightarrow b$ TO $t \rightarrow s$}

Ordinarily the weak transition $t \rightarrow s$ is suppressed relative to $t \rightarrow b$ by the ratio of the relevant Kobayashi-Maskawa matrix elements squared, ${ }^{11}\left|V_{t s}\right|^{2} /\left|V_{t b}\right|^{2} \approx$ 1/500. However, we have seen that $\Gamma\left(t \rightarrow b e^{+} \nu_{e}\right)$ increases sharply as $m_{t}$ crosses the $W$ threshold, changing from being proportional to $G_{F}^{2}$ to being proportional to $G_{F}$. Thus we expect $\Gamma\left(t \rightarrow s e^{+} \nu_{e}\right)$ to be enhanced relative to $\Gamma\left(t \rightarrow b e^{+} \nu_{e}\right)$ when $m_{t}$ lies between the two thresholds: $M_{W}+m_{s}<m_{t}<M_{W}+m_{b}$. The question is whether the threshold enhancement "wins" over the Kobayashi-Maskawa suppression.

To examine this quantitatively we consider the ratio of the widths with the 


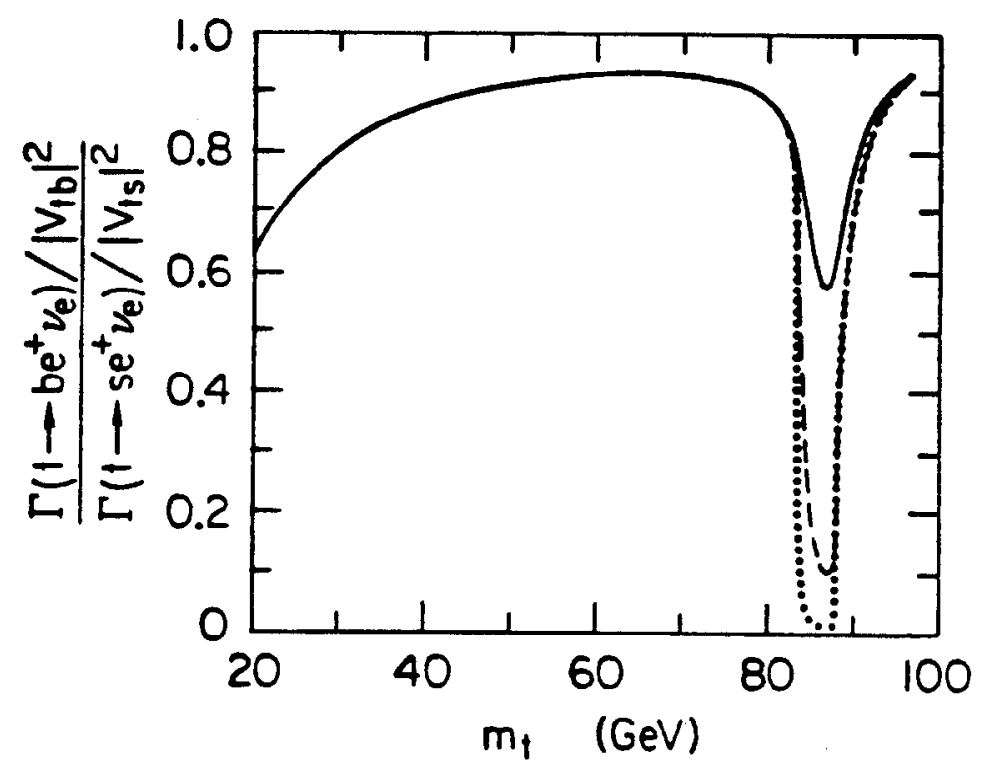

Figure 2.4 The ratio of decay rates with Kobayashi-Maskawa factors taken out, $\left(\Gamma\left(t \rightarrow b e^{+} \nu_{e}\right) /\left|V_{t b}\right|^{2}\right) /\left(\Gamma\left(t \rightarrow s e^{+} \nu_{e}\right) /\left|V_{t s}\right|^{2}\right)$ with $m_{b}=5 \mathrm{GeV}$ and $m_{s}=0.5 \mathrm{GeV}$ and $\Gamma_{w}$ equal to fictitious values of $0.0225 \mathrm{GeV}$ (dotted curve) and $0.225 \mathrm{GeV}$ (dashed curve), and the expected $2.25 \mathrm{GeV}$ (solid curve).

Kobayashi-Maskawa factors divided out:

$$
\frac{\Gamma\left(t \rightarrow b e^{+} \nu_{e}\right) /\left|V_{t b}\right|^{2}}{\Gamma\left(t \rightarrow s e^{+} \nu_{e}\right) /\left|V_{t s}\right|^{2}}
$$

Either well below or well above threshold for a "real" $W$ this ratio should be near unity. For an infinitely narrow $W$ the denominator is strongly enhanced, but the numerator is not, when $M_{w}+m_{s}<m_{t}<M_{w}+m_{b}$. The ratio indeed drops dramatically near $t \rightarrow s+W$ threshold, as shown in Figure 2.4, for $\Gamma_{W}=$ $0.0225 \mathrm{GeV}$ (dotted curve) and even for $\Gamma_{w}=0.225 \mathrm{GeV}$ (dashed curve). However, the expected $W$ width of $2.25 \mathrm{GeV}$ (solid curve) smears out the threshold effect over a mass range that is of the same order as $m_{b}-m_{s}$, and gives only a modest $\operatorname{dip}($ to $\approx 0.6)$ in the ratio. This is hardly enough to make $t \rightarrow s$ comparable to $t \rightarrow b$. 


\subsection{Exclusive Modes}

When $m_{t}$ is in the present experimentally acceptable range, the rate for weak decay of the constituent $t$ quarks within possible hadrons becomes comparable with that for electromagnetic and weak decays. Weak decays of toponium become a major fraction of, say, the $J^{P}=1^{-}$ground state, and even for the $T^{*}(t \bar{q})$ vector meson, weak decays can dominate the radiative magnetic dipole transition to its hyperfine partner, the $\mathrm{T}$ meson $J^{P}=0^{-}$ground state. ${ }^{13}$

In decays of heavy flavor mesons the branching ratios for typical exclusive channels scale like $\left(f / M_{Q}\right)^{2}$, where $f$ is a meson decay constant (like $f_{\pi}$ or $f_{K}$ ), of order $100 \mathrm{MeV}$, and $M_{Q}$ is the mass of the heavy quark. For $D$ mesons individual channels have branching ratios of a few percent; for $B$ mesons they are roughly ten times smaller; and for $T$ (or $T^{*}$ ) mesons they should be a hundred or more times smaller yet. It should be possible to treat $T$ decays in terms of those of the constituent $t$ quark, $t \rightarrow b+W^{+}$, with the $b$ quark appearing in a $b$ jet not so different from those already observed at PEP and PETRA.

There is one possible exception to these last statements, and that is when $m_{t} \approx m_{b}+M_{W}$, the situation under study here. In this case there is a premium on giving as much energy to the $W$ as possible, i.e., keeping as far above threshold for "real" $W$ production as possible, and hence on keeping the invariant mass of the hadronic system containing the $b$ quark small. Then we expect the $T$ and $T^{*}$ to decay dominantly into a few exclusive channels: a "real" $W$ plus a $B$ or a "real" $W$ plus a $B^{*}$.

Furthermore, this is one place where the use of the non-relativistic quark model 
is a priori well-justified. The $t$ quark and final $W$ are very heavy. When $m_{t} \approx$ $m_{b}+M_{w}$, the final heavy $b$ quark is restricted to have a few $\mathrm{GeV}$ or less of kinetic energy if the $W$ is to be as "real" as possible. The accompanying light quark in the $T$ hadron is very much a spectator which simply becomes part of the final $B$ or $B^{*}$ hadron. Thus we can match up the weak current of heavy quark states,

$$
\left\langle b\left(p_{b}, \lambda_{b}\right)\left|V^{\mu}-A^{\mu}\right| t\left(p_{t}, \lambda_{t}\right)\right\rangle=\bar{u}\left(p_{b}, \lambda_{b}\right) \gamma^{\mu}\left(1-\gamma^{5}\right) u\left(p_{t}, \lambda_{t}\right)
$$

sandwiched between the appropriate hadronic wavefunctions in spin and flavor space, with the matrix elements of the exclusive hadronic channels $T \rightarrow B+W$ and $T \rightarrow B^{*}+W$, defined in terms of the form factors ${ }^{14-16}$

$$
\left\langle B\left(p_{B}\right)\left|V^{\mu}\right| T\left(p_{T}\right)\right\rangle=f_{+}\left(p_{T}^{\mu}+p_{B}^{\mu}\right)+f_{-}\left(p_{T}^{\mu}-p_{B}^{\mu}\right)
$$

and

$$
\begin{aligned}
& \left\langle B^{*}\left(p_{B}, \epsilon\right)\left|V^{\mu}-A^{\mu}\right| T\left(p_{T}\right)\right\rangle= \\
& \quad a \epsilon^{\mu *}+b\left(p_{T} \cdot \epsilon^{*}\right)\left(p_{T}+p_{B}\right)^{\mu}+c\left(p_{T} \cdot \epsilon^{*}\right)\left(p_{T}-p_{B}\right)^{\mu}+i g \epsilon_{\alpha \beta \gamma}^{\mu} p_{T}^{\alpha} p_{B}^{\beta} \epsilon^{\gamma *} .
\end{aligned}
$$

The quark-level calculation is carried out using explicit spinors and $\gamma$-matrices in the rest frame of the $t$ :

$$
\begin{aligned}
& u\left(p_{t}, \lambda\right)=\sqrt{2 m_{t}}\left[\begin{array}{c}
\chi_{\lambda} \\
0
\end{array}\right], \\
& u\left(p_{b}, \lambda\right)=\sqrt{E_{b}+m_{b}}\left[\begin{array}{c}
\chi_{\lambda} \\
\frac{\lambda|\mathbf{P}|}{E_{b}+m_{b}} \chi_{\lambda}
\end{array}\right],
\end{aligned}
$$


with

$$
\chi_{+}=\left[\begin{array}{l}
1 \\
0
\end{array}\right], \quad \chi_{-}=\left[\begin{array}{l}
0 \\
1
\end{array}\right],
$$

and where $E_{b}$ is the energy of the $b$-quark and $\mathbf{P}=-\mathbf{Q}_{w}$ is its momentum. Evaluating the matrix elements from Eq. (2.7) yields

$$
\begin{aligned}
& \left\langle b\left(p_{b}, \lambda_{b}\right)\left|V^{\mu}\right| t\left(p_{t}, \lambda_{t}\right)\right\rangle= \\
& \sqrt{\frac{2 m_{t}}{E_{b}+m_{b}}}|\mathrm{p}|\left\{\begin{array}{ccccc}
\left(E_{b}+m_{b}\right) /|\mathrm{p}|, & 0, & 0, & 1 ; & \lambda_{t} \lambda_{b}=++,--; \\
0, & -1, & -i, & 0 ; & \lambda_{t} \lambda_{b}=+-; \\
0, & 1, & -i, & 0 ; & \lambda_{t} \lambda_{b}=-+;
\end{array}\right.
\end{aligned}
$$

and

$$
\begin{gathered}
\left\langle b\left(p_{b}, \lambda_{b}\right)\left|A^{\mu}\right| t\left(p_{t}, \lambda_{t}\right)\right\rangle= \\
\sqrt{2 m_{t}\left(E_{b}+m_{b}\right)}\left\{\begin{array}{ccccc}
|\mathbf{p}| /\left(E_{b}+m_{b}\right), & 0, & 0, & 1 ; & \lambda_{t} \lambda_{b}=++; \\
0, & 1, & i, & 0 ; & \lambda_{t} \lambda_{b}=+-; \\
0, & 1, & -i, & 0 ; & \lambda_{t} \lambda_{b}=-+; \\
-|\mathbf{p}| /\left(E_{b}+m_{b}\right), & 0, & 0, & -1 ; & \lambda_{t} \lambda_{b}=--
\end{array}\right.
\end{gathered}
$$

We choose the polarization vectors of the $W$ to be:

$$
\begin{aligned}
\epsilon_{ \pm}^{\mu} & =-\frac{1}{\sqrt{2}}(0, \pm 1, i, 0), \\
\epsilon_{L}^{\mu} & =\frac{1}{M_{W}}\left(-|\mathrm{p}|, 0,0, E_{W}\right),
\end{aligned}
$$

where $E_{W}$ is the energy of the $W$. To obtain the amplitudes we dot the currents from Eqs. (2.11) and (2.12) with the polarization vectors:

$$
\begin{aligned}
& \mathcal{M}_{\lambda_{t} \lambda_{b}}^{\nu}=\left\langle b\left(p_{b}, \lambda_{b}\right)\left|V_{\mu}\right| t\left(p_{t}, \lambda_{t}\right)\right\rangle \epsilon_{\lambda}^{\mu *}, \\
& \mathcal{M}_{\lambda_{t} \lambda_{b}}^{A}=\left\langle b\left(p_{b}, \lambda_{b}\right)\left|A_{\mu}\right| t\left(p_{t}, \lambda_{t}\right)\right\rangle \epsilon_{\lambda}^{\mu *} .
\end{aligned}
$$

The appropriate polarization vector to go with each spin configuration is chosen 
by angular momentum conservation. For the vector current we find

$$
\begin{aligned}
& \mathcal{M}_{+-}^{v}=M_{-+}^{v}=-2 \sqrt{\frac{m_{t}}{E_{b}+m_{b}}}|\mathbf{p}|, \\
& \mathcal{M}_{++}^{v}=M_{--}^{v}=\sqrt{\frac{2 m_{t}}{E_{b}+m_{b}}} \frac{|\mathbf{p}|}{M_{W}}\left(m_{t}+m_{b}\right),
\end{aligned}
$$

and for the axial current

$$
\begin{aligned}
& \mathcal{M}_{+-}^{A}=-M_{-+}^{A}=-2 \sqrt{\frac{\pi_{t}}{E_{b}+m_{b}}}|\mathrm{p}| \\
& \mathcal{M}_{+.+}^{A}=-M_{--}^{A}=2 \sqrt{m_{t}\left(E_{b}+m_{b}\right)}
\end{aligned}
$$

The quark model results for the amplitudes for $T \rightarrow B W$ and $T \rightarrow B^{*} W$ are obtaincd by sandwiching the quark level results between the appropriate wave functions in spin and flavor:

$$
\begin{aligned}
& |T\rangle=\frac{1}{\sqrt{2}}|t \uparrow \bar{q} \downarrow-t \downarrow \bar{q} \uparrow\rangle, \\
& |B\rangle=\frac{1}{\sqrt{2}}|b \uparrow \bar{q} \downarrow-b \downarrow \bar{q} \uparrow\rangle, \\
& \left|B_{\lambda=+1}^{*}\right\rangle=|b \uparrow \bar{q} \uparrow\rangle, \\
& \left|B_{\lambda=-1}^{*}\right\rangle=|b \downarrow \bar{q} \downarrow\rangle, \\
& \left|B_{\lambda=0}^{*}\right\rangle=\frac{1}{\sqrt{2}}|b \uparrow \bar{q} \downarrow+b \downarrow \bar{q} \uparrow\rangle,
\end{aligned}
$$

where $q$ is a light quark: $q=u, d$, or $s$.

We can now write down the quark model results. The decay $T \rightarrow B W$ yields only longitudinally polarized $W$ 's, by angular momentum conservation. By parity, 
it proceeds only through the vector current:

$$
\begin{aligned}
\mathcal{M}\left(T \rightarrow B W_{L}\right) & =\frac{1}{2}\left(\mathcal{M}_{++}^{v}+\mathcal{M}_{--}^{v}\right) \\
& =\sqrt{\frac{2 m_{t}}{E_{b}+m_{b}}} \frac{|\mathbf{p}|}{M_{W}}\left(m_{t}+m_{b}\right) .
\end{aligned}
$$

The decay of a $T$ into a transverse $B^{*}$ involves both the axial and vector currents, whereas the decay into a longitudinal $B^{*}$ involves only the axial current:

$$
\begin{aligned}
\mathcal{M}\left(T \rightarrow B_{\lambda=+1}^{*} W_{\lambda=+1}\right) & =-\frac{1}{\sqrt{2}}\left(\mathcal{M}_{-+}^{v}+\mathcal{M}_{-+}^{A}\right) \\
& =\sqrt{\frac{2 m_{t}}{E_{b}+m_{b}}}\left(E_{b}+m_{b}+|\mathbf{p}|\right), \\
\mathcal{M}\left(T \rightarrow B_{\lambda=-1}^{*} W_{\lambda=-1}\right) & =\frac{1}{\sqrt{2}}\left(\mathcal{M}_{-+}^{v}+\mathcal{M}_{-+}^{A}\right) \\
& =\sqrt{\frac{2 m_{t}}{E_{b}+m_{b}}}\left(E_{b}+m_{b}-|\mathbf{p}|\right), \\
\mathcal{M}\left(T \rightarrow B_{L}^{*} W_{L}\right) & =\frac{1}{2}\left(\mathcal{M}_{++}^{A}+\mathcal{M}_{--}^{A}\right) \\
& =\frac{\left(m_{t}-m_{b}\right)}{M_{W}} \sqrt{m_{t}\left(E_{b}+m_{b}\right)} .
\end{aligned}
$$

The corresponding quantities in terms of the form factors are computed by dotting the polarization vectors into Eqs. (2.8) and (2.9):

$$
\begin{aligned}
\mathcal{M}\left(T \rightarrow B+W_{L}\right) & =2 \frac{m_{T}}{M_{W}}|\mathbf{p}| f_{+} \\
\mathcal{M}\left(T \rightarrow B^{*}+W_{T}\right) & =\left(a+\lambda_{W} g m_{T}|\mathbf{p}|\right) \\
\mathcal{M}\left(T \rightarrow B^{*}+W_{L}\right) & =\frac{1}{M_{W} m_{B^{*}}}\left[a\left(|\mathbf{p}|^{2}+E_{B^{*}} E_{W}\right)+2 b m_{T}^{2}|\mathbf{p}|^{2}\right]
\end{aligned}
$$

Identifying $m_{t}=m_{T}$ and $m_{b}=m_{B}=m_{B^{*}}$ and comparing the quark level and 
hadron level expressions:

$$
\begin{aligned}
f_{+} & =\frac{m_{T}+m_{B}}{\sqrt{2 m_{T}\left(E_{B}+m_{B}\right)}} \\
a & =\sqrt{2 m_{T}\left(E_{B}+m_{B}\right)} \\
g & =\frac{2}{\sqrt{2 m_{T}\left(E_{B}+m_{B}\right)}} \\
b & =\sqrt{\frac{E_{B}+m_{B}}{2 m_{T}}} \frac{\left(m_{B}-E_{B}\right)}{|\mathbf{p}|^{2}} .
\end{aligned}
$$

In the limit $|\mathbf{p}| \rightarrow 0$ the form factors reduce to

$$
\begin{aligned}
f_{+} & =\frac{m_{T}+m_{B}}{2 \sqrt{m_{T} m_{B}}} \\
a & =2 \sqrt{m_{T} m_{B}} \\
g & =\frac{1}{\sqrt{m_{T} m_{B}}} \\
b & =\frac{-1}{2 \sqrt{m_{T} m_{B}}} .
\end{aligned}
$$

These results agree in the appropriate limit with previous results ${ }^{13-15}$. The form factors $f_{+}, a$, and $g$ all have straightforward limits as $|\mathbf{p}| \rightarrow 0$, while that for $b$ can be subtle, as explicitly seen in Eq. (2.23). It is more sensitive to bound quarks being off the mass-shell. ${ }^{15}$ Our result agrees with that of Ref. 16 with the appropriate change of flavors.

\subsection{W POlarization in $t$ DecaY}

Within the scenario of discovery of the top quark at a hadron collider, it would be useful to have several handles on the value of $m_{t}$. An indirect method would be to measure a quantity in top decays which depends strongly on the top mass. 
For $m_{t}$ in the vicinity of $M_{w}+m_{b}$, we now show that such a quantity is the ratio of the production of longitudinal $W$ 's to that of transverse $W$ 's in top decay.

The decay widths into longitudinal and transverse $W$ 's are defined by decomposing the numerator of the $W$ propagator as

$$
g_{\mu \nu}-Q_{\mu} Q_{\nu} / M_{w}^{2}=\sum_{\lambda} \epsilon_{\mu}(\lambda) \epsilon_{\nu}^{\star}(\lambda)=\epsilon_{\mu}^{(+)} \epsilon_{\nu}^{(+) \star}+\epsilon_{\mu}^{(0)} \epsilon_{\nu}^{(0) \star}+\epsilon_{\mu}^{(-)} \epsilon_{\nu}^{(-) \star},
$$

where the superscripts give the helicity of the $W$, whether virtual or real. In calculating the $t$ decay rate in Eq. (2.1), we define $\Gamma_{L}=\Gamma^{(0)}$, originating from $W^{\prime} \mathrm{s}$ with helicity zero, and $\Gamma_{T}=\Gamma^{(+)}+\Gamma^{(-)}$, originating from $W$ 's with helicity \pm 1 . There is no interference between amplitudes involving the different $W$ helicities, since the helicity of the $t$ and $b$ quarks determines the helicity of the intermediate $W$. Separating in this way the portions of Eq. (2.1) originating from longitudinal and transverse $W$ 's, we find

$$
\begin{gathered}
\Gamma_{L}=\frac{G_{F}^{2} m_{t}^{5}}{24 \pi^{3}} \int_{0}^{\left(m_{t}-m_{b}\right)^{2}} d Q^{2} \frac{M_{W}^{4}|\mathbf{Q}|}{\left(Q^{2}-M_{W}^{2}\right)^{2}+M_{W}^{2} \Gamma_{W}^{2}}\left[2|\mathbf{Q}|^{2}+Q^{2}\left(1-\frac{Q_{0}}{m_{t}}\right)\right], \\
\Gamma_{T}=\frac{G_{F}^{2} m_{t}^{5}}{24 \pi^{3}} \int_{0}^{\left(m_{t}-m_{b}\right)^{2}} d Q^{2} \frac{M_{W}^{4}|\mathbf{Q}|}{\left(Q^{2}-M_{W}^{2}\right)^{2}+M_{W}^{2} \Gamma_{W}^{2}}\left[2 Q^{2}\left(1-\frac{Q_{0}}{m_{t}}\right)\right] .
\end{gathered}
$$

In the case $m_{t}<<M_{W}$ the integrals become

$$
\begin{aligned}
& \Gamma_{L}=\frac{G_{F}^{2} m_{t}^{5}}{24 \pi^{3}} \int_{0}^{\left(m_{t}-m_{b}\right)^{2}} d Q^{2}|\mathbf{Q}|\left[2|\mathbf{Q}|^{2}+Q^{2}\left(1-\frac{Q_{0}}{m_{t}}\right)\right], \\
& \Gamma_{T}=\frac{G_{F}^{2} m_{t}^{5}}{24 \pi^{3}} \int_{0}^{\left(m_{\mathfrak{t}}-m_{b}\right)^{2}} d Q^{2}|\mathbf{Q}|\left[2 Q^{2}\left(1-\frac{Q_{0}}{m_{t}}\right)\right] .
\end{aligned}
$$


Noticing that

$$
\frac{d|\mathbf{Q}|}{d Q^{2}}=\frac{1}{2|\mathbf{Q}|}\left(-1+\frac{Q_{0}}{m_{t}}\right)
$$

and using integration by parts, we find

$$
\begin{aligned}
& \Gamma_{L}=\frac{G_{F}^{2} m_{t}^{5}}{18 \pi^{3}} \int_{0}^{\left(m_{t}-m_{b}\right)^{2}} d Q^{2} 2|\mathbf{Q}|^{3}, \\
& \Gamma_{T}=\frac{G_{F}^{2} m_{t}^{5}}{18 \pi^{3}} \int_{0}^{\left(m_{t}-m_{b}\right)^{2}} d Q^{2}|\mathbf{Q}|^{3} .
\end{aligned}
$$

Without needing to perform the integrals we see that

$$
\frac{\Gamma_{L}}{\Gamma_{T}}=2
$$

Sufficiently far above the $W$ threshold we need only calculate the relative production of longitudinal and transverse real $W$ 's:

$$
\frac{\Gamma_{L}}{\Gamma_{T}}=\frac{1}{2}+\frac{m_{t}\left|\mathbf{Q}_{W}\right|^{2}}{E_{b} M_{W}^{2}}
$$

As $m_{t}$ gets very large the longitudinal piece dominates because its coupling grows like $\left(m_{t} / M_{W}\right)^{2}$. For the case of an infinitely narrow $W, \Gamma_{L} / \Gamma_{T}=\frac{1}{2}$, precisely at threshold. At the threshold the decay is purely $s$-wave and the three polarization states are produced equally. The value of $\Gamma_{L} / \Gamma_{T}$ near the threshold is shown in Figure 2.5 for $\Gamma_{W}=0.0225 \mathrm{GeV}$ (dotted curve), $0.225 \mathrm{GeV}$ (dashed curve), and the expected $2.25 \mathrm{GeV}$ (solid curve). In this case we see that even for the expected value of $\Gamma_{W}$ the ratio varies rapidly with $m_{t}$, especially just below the threshold. 


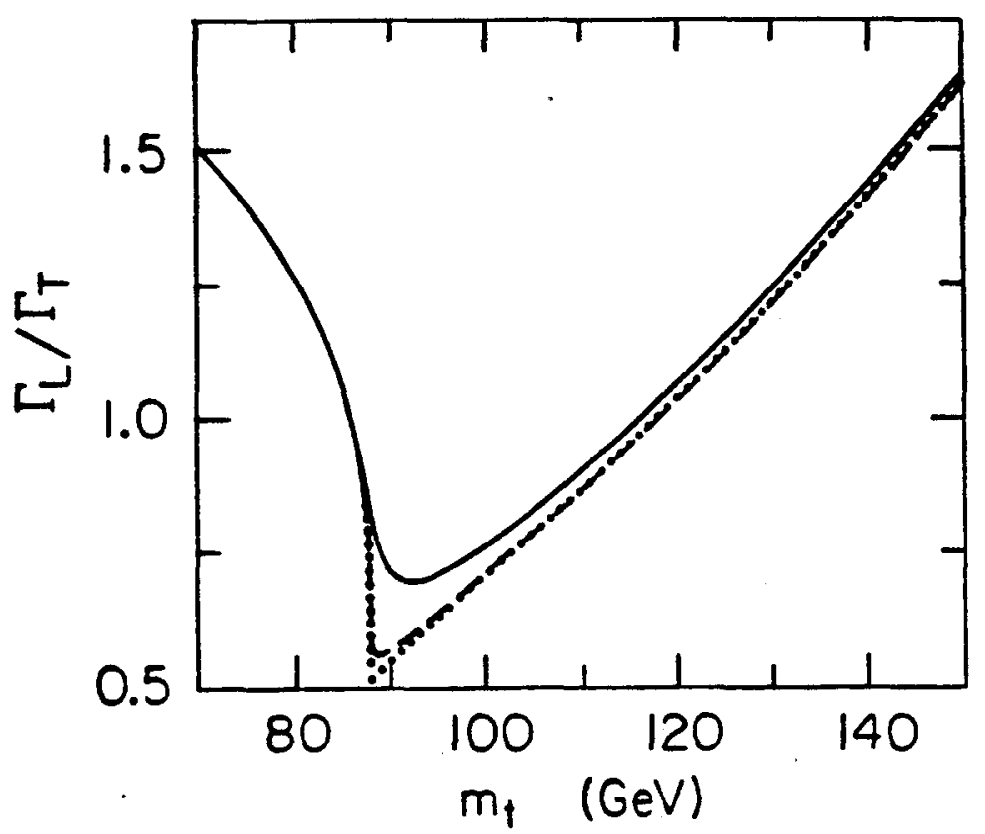

Figure 2.5 The ratio $\Gamma_{L} / \Gamma_{T}$ of $t \rightarrow b+W \rightarrow b e^{+} \nu_{e}$ decay widths into longitudinal compared to transverse $W$ 's as a function of $m_{t}$ for $\Gamma_{W}$ equal to fictitious values of $0.0225 \mathrm{GeV}$ (dotted curve) and $0.225 \mathrm{GeV}$ (dashed curve), and the expected $2.25 \mathrm{GeV}$ (solid curve).

The ratio of longitudinal to transverse $W$ 's is reflected in the angular distribution of the electrons ${ }^{17}$ from its decay. With the final $b$ quark direction as a polar axis,

$$
\frac{d \Gamma}{d \cos \theta}=1+\beta \cos \theta+\alpha \cos ^{2} \theta
$$

where

$$
\alpha=\frac{\Gamma_{T}-\Gamma_{L}}{\Gamma_{T}+\Gamma_{L}}
$$

Thus a measurement of the piece of the angular distribution even in $\theta$ gives a value for $\Gamma_{L} / \Gamma_{T}$ and indirectly a value for $m_{t}$. In particular, $\alpha$ becomes positive only a few $\mathrm{GeV}$ below the threshold, and this may provide a useful lower bound on $m_{t}$.

The coefficient of $\cos \theta$ contains information on the difference between the two 
transverse polarizations:

$$
\beta=2\left[\frac{\Gamma_{(-)}-\Gamma_{(+)}}{\Gamma_{L}+\Gamma_{T}}\right]
$$

If the energy of the $b$ quark is much larger than its mass then the $b$ will be lefthanded, since its coupling to the $W$ is $V-A$. Thus if the spin of the top quark is aligned along the $W$ momentum then the $W$ will be dominantly longitudinal; if the top spin is anti-aligned with the $W$ momentum then the $W$ will prefer negative helicity. So between the two transverse states the negative helicity state will dominate when the $b$ energy is high. Indeed, in the case of a massless $b$ the positive helicity state would not be produced at all. However at the threshold for making a real $W, m_{b} / E_{b}$ is no suppression at all and the two transverse states are produced equally. To see how this comes about consider the difference of the two transverse rates divided by their sum:

$$
\frac{\Gamma_{(-)}-\Gamma_{(+)}}{\Gamma_{(-)}+\Gamma_{(+)}} .
$$

For top masses sufficiently far away from the threshold this ratio will be close to one as the positive helicity piece will be suppressed. At exactly the threshold the ratio goes to zero, in the limit that the $W$ is infinitely narrow. The results for the expected width of the $W$ are shown in Figure 2.6, along with two fictitious choices of the width for comparison. We see that for very small values of the width the ratio becomes very small near the threshold, but for the expected width the effect is slight, the ratio achieving a minimum of $\sim 0.8$. 


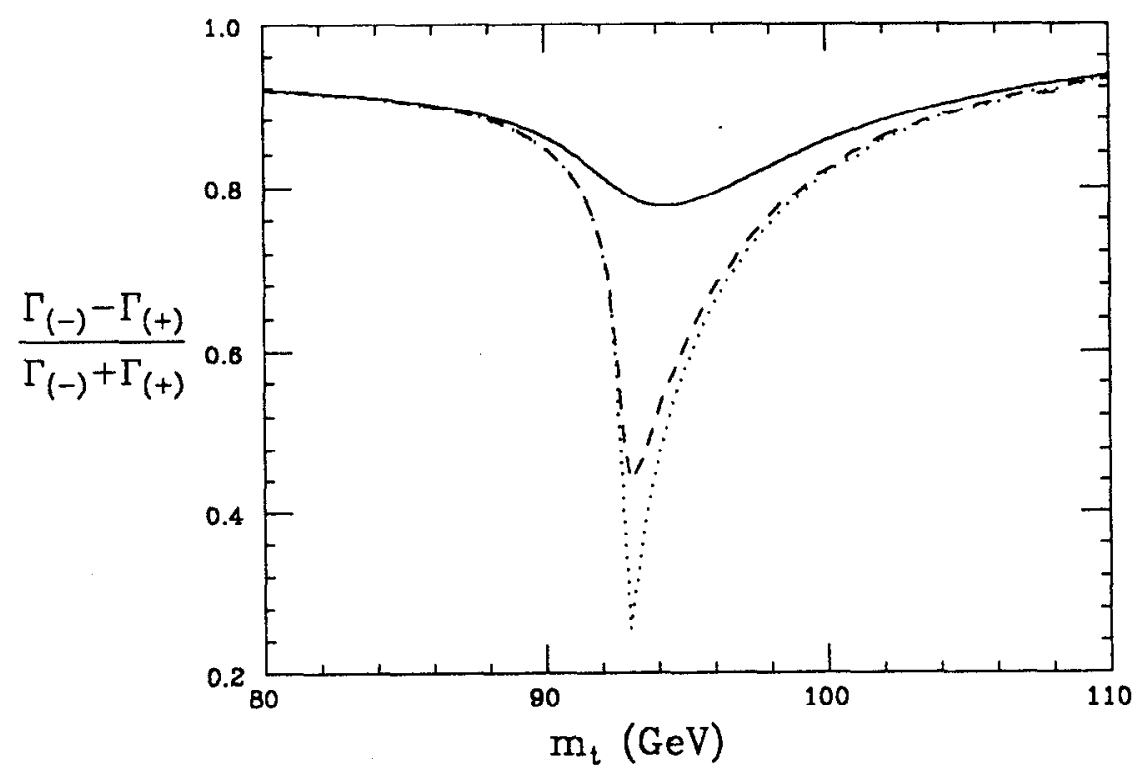

Figure 2.6 The ratio $\left(\Gamma_{(-)}-\Gamma_{(+)}\right) /\left(\Gamma_{(-)}+\Gamma_{(+)}\right)$of $t \rightarrow b+W \rightarrow b e^{+} \nu_{e}$ decay widths into left-handed minus right-handed $W$ 's divided by the sum as a function of $m_{t}$ for $\Gamma_{W}$ equal to fictitious values of $0.0225 \mathrm{GeV}$ (dotted curve) and $0.225 \mathrm{GeV}$ (dashed curve), and the expected $2.25 \mathrm{GeV}$ (solid curve).

\subsection{Summary and Conclusions}

We have seen that the range of top masses near the threshold for production of real $W$ bosons has a rich structure. Both the absolute width of the top quark and its differential width in $Q^{2}$ vary wildly across this region. Top quarks in this region have a slightly enhanced decays into strange quarks. The exclusive decay rates for top mesons can calculated around the threshold using the non-relativistic quark model. The relative populations of the different polarizations of intermediate $W$ in top quark decays changes rapidly in this region and could provide information on the top quark mass. 


\section{Top Quark Production in $e^{+} e^{-}$Colliders}

\subsection{INTRODUCTION}

In the last several years much thought has gone into the prospects for physics using an $e^{+} e^{-}$collider with energy in the $\mathrm{TeV}$ range. ${ }^{18}$ These machines show great promise for being able to address a wide range of experimental issues. These include (but are not limited to) Higgs boson searches, $W$-pair production, supersymmetry searches, and charged Higgs searches. Here we wish to study the production of top quarks in a $e^{+} e^{-}$collider with a total center-of-mass energy of order $1 \mathrm{TeV}$. Top quarks, besides being of considerable interest in their own right, provide signals and/or backgrounds in all the aforementioned experiments. In particular, since Higgs bosons couple predominantly to the most massive particle available, top quarks will figure prominently in any Higgs study, whether for a charged or neutral Higgs.

The scale of cross sections for all Standard Model processes (and most nonstandard ones) is set by the elementary QED point cross section:

$$
\sigma_{\mathrm{pt}}=\frac{4 \pi \alpha^{2}}{3 s}=\frac{86.8 \mathrm{fb}}{[E(\mathrm{TeV})]^{2}}
$$

The canonical production mechanism for fermion pair production is $e^{+} e^{-}$annihilation into a photon or $Z^{0}$, shown in Figure 3.1. At center-of-mass energies much larger than the $Z^{0}$ mass the lowest order cross-section for a fermion, $f$, can be written

$$
\sigma\left(e^{+} e^{-} \rightarrow f \bar{f}\right)=\sigma_{\mathrm{pt}} N_{c} \frac{\left[10 Q^{2} s_{w}^{4}+2 Q I_{3} s_{w}^{2}\left(1-6 s_{w}^{2}\right)+1-4 s_{w}^{2}+8 s_{w}^{4}\right]}{16 s_{W}^{4}\left(1-s_{w}^{2}\right)^{2}},
$$

where $I_{3}$ is the weak isospin of the fermion, $Q$ its charge, and $N_{c}$ is the number of 


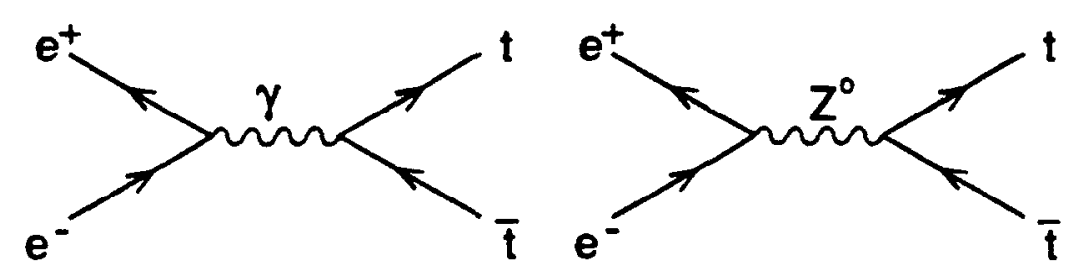

Figure 3.1 The Feynman diagrams for $e^{+} e^{-} \rightarrow \gamma, Z^{0} \rightarrow t \bar{t}$.

colors, 1 for leptons, 3 for quarks. For compactness we have written $\sin \theta_{W}=s_{W}$. For top quarks (or any other up-type quark for that matter) the cross section is

$$
\sigma\left(e^{+} e^{-} \rightarrow t \bar{t}\right) \simeq 2.1 \sigma_{\mathrm{pt}}
$$

where we have assumed $s \gg m_{t}^{2}, M_{z}^{2}$ and taken $s_{W}^{2}=0.23$.

The question we want to investigate is whether there are any other important production mechanisms for top quarks. The natural candidates are the vector boson fusion processes. These processes are suppressed relative to $\sigma_{\mathrm{pt}}$ by a factor of $\alpha^{2}$. However, since the vector bosons are exchanged in the $t$-channel they can come close to being on shell in the limit where the energy they carry is much larger than their mass. Furthermore, longitudinal bosons have enhanced couplings to heavy fermions. These factors could combine to make vector-boson fusion processes competitive with annihilation through $\gamma$ or $Z^{0}$.

In this chapter we will make an exhaustive survey of the vector-boson fusion processes which contribute to the production of top quarks. We will calculate cross sections for these processes using the effective-vector-boson approximation. For 
definiteness we will present results for a collider with a center-of-mass energy of 2 $\mathrm{TeV}$.

\subsection{The Effective-Photon Approximation}

Among the vector-boson fusion processes, the one with the longest history by far is photon-photon fusion, with theoretical investigations going all the way back to Williams and Landau and Lifshitz ${ }^{19}$ in 1934. At the energies in which we are interested, these reactions are well described by the effective-photon approximation, originally developed by Fermi, Weizsäcker and Williams, and Landau and

Lifshitz, ${ }^{20}$ and given a modern treatment by Brodsky, Kinoshita and Terazawa. ${ }^{21}$ For completeness and as a warm-up for subsequent calculations, we will present a brief derivation of the effective-photon approximation, keeping only the leading term. In this strategy we will neglect the mass of the electron wherever possible. We will follow closely the treatment of Ref. 21 .

Consider the process depicted in Figure 3.2. The essence of the effective-photon approximation is that the cross section is dominated by phase space configurations in which the virtual photon is nearly on-shell, i.e., when the final electron goes almost straight forward. The strategy is to integrate over forward angles and express the result as the cross section for $\gamma f \rightarrow X$ multiplied by an effective flux of photons inside the electron. In this respect the effective-photon approximation is identical to the parton model of hadrons, the difference being that the distribution of photons inside the electron is calculable, whereas the distribution of quarks and gluons within a hadron must be extracted from experimental data. 


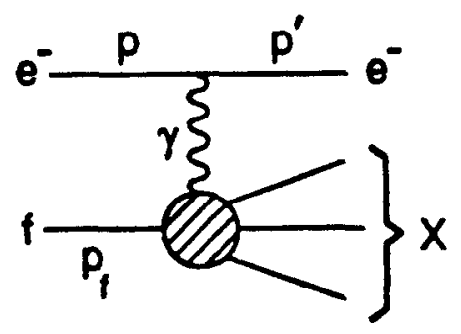

Figure 3.2 One-photon production of an arbitrary final state $X$ in a collision of an electron with an arbitrary initial particle $f: e^{-} f \rightarrow e^{-} X$.

The amplitude for this process can be written

$$
\mathcal{M}=\bar{u}\left(p^{\prime}\right)\left(i e \gamma^{\mu}\right) u(p) \frac{\left(-i g_{\mu \nu}\right)}{\left(p-p^{\prime}\right)^{2}} \mathcal{A}^{\nu}
$$

where $\mathcal{A}^{\nu}$ describes the three-point coupling, $\gamma^{*} f \rightarrow X$. After summing over the helicities of the initial and final electron and performing the Dirac trace we have

$$
\frac{1}{2} \sum_{\text {spins }}|\mathcal{M}|^{2}=\frac{2 e^{2}}{\left(p-p^{\prime}\right)^{4}}\left(p^{\mu} p^{\prime \nu}+p^{\nu} p^{\prime \mu}-p \cdot p^{\prime} g^{\mu \nu}\right) \mathcal{A}_{\mu} \mathcal{A}_{\nu}^{\dagger}
$$

Proceed to the cross section:

$$
\begin{gathered}
d \sigma\left(e^{-} f \rightarrow e^{-} X\right)=\frac{\alpha}{2 \pi^{2}} \int \frac{d^{3} p^{\prime}}{E^{\prime}}\left(2 p^{\mu} p^{\alpha}+\frac{1}{2} k^{2} g^{\mu \alpha}\right) \frac{g_{\mu \nu}}{k^{2}} \frac{g_{\alpha \beta}}{k^{2}} \mathcal{A}^{\nu} \mathcal{A}^{\beta \dagger} d \tilde{\Gamma} \\
d \tilde{\Gamma}=\frac{(2 \pi)^{4}}{2 s} \delta\left(k+p_{f}-p_{X}\right) d \Gamma
\end{gathered}
$$

where $s=\left(p+p_{f}\right)^{2}, k=p-p^{\prime}$, is the momentum of the photon and $d \Gamma$ is the invariant phase space of the state $X$. 
We now break up the photon propagator into a sum of polarization vectors:

$$
-g^{\mu \nu} \rightarrow \sum_{i} \epsilon_{i}^{\mu} \epsilon_{i}^{* \nu}
$$

The sum runs over the two polarization vectors perpendicular to the photon momentum $\mathbf{k}$ plus a longitudinal one. Inserting Eq. (3.3) into Eq. (3.1) yields

$$
d \sigma(e f \rightarrow e X)=\frac{\alpha}{2 \pi^{2}} \int \frac{d^{3} p^{\prime}}{E^{\prime} k^{4}} \sum_{i j}\left[2\left(p \cdot \epsilon_{i}\right)\left(p \cdot \epsilon_{j}^{*}\right)+\frac{1}{2}\left(\epsilon_{i} \cdot \epsilon_{j}^{*}\right) k^{2}\right]\left(\mathcal{A} \cdot \epsilon_{i}\right)\left(\mathcal{A}^{\dagger} \cdot \epsilon_{j}^{*}\right) d \tilde{\Gamma} .
$$

The integral over the azimuthal angle of the final electron causes the polarization sum to be diagonal; the interference terms between longitudinal and transverse polarizations and between the two transverse polarizations integrate to give zero. Furthermore, the contribution from the longitudinal polarization vectors is suppressed. The longitudinal polarization vector becomes proportional to $k$ when the photon goes on shell. Thus the Ward identity guarantees that it couples with an extra factor of $k^{2}$ compared to the transverse mode. The longitudinal piece will only be important if it is anomalously enhanced, e.g., by a small mass appearing in a propagator, or if the transverse coupling is forbidden; the longitudinal piece will not contribute significantly to $\gamma \gamma$ production of fermion pairs, and we neglect it in what follows.

After the azimuthal integration is performed, we have

$$
d \sigma(e f \rightarrow e X)=\frac{\alpha}{\pi} \int \frac{E^{\prime} d E^{\prime} d \cos \theta^{\prime}}{k^{4}}\left(2 p_{\perp}^{2}-k^{2}\right) \frac{\omega}{E} d \sigma(\gamma f \rightarrow X),
$$

where $\omega=E-E^{\prime}$ is the energy of the photon and $p_{\perp}$ is the projection of $\mathrm{p}$ 
perpendicular to k. In writing Eq. (3.4) we have made the substitution

$$
\lim _{k^{2} \rightarrow 0} \sum_{i=1,2}\left(\mathcal{A} \cdot \epsilon_{i}\right)\left(\mathcal{A}^{\dagger} \cdot \epsilon_{i}^{*}\right) d \tilde{\Gamma}=2 \omega \cdot d \sigma(\gamma f \rightarrow X)
$$

The remaining integral over the polar angle of the final electron is peaked sharply around $\theta=0$ so we replace

$$
\begin{gathered}
k^{2} \simeq-2 E E^{\prime}(1-\cos \theta)-m_{e}^{2} \frac{\left(E-E^{\prime}\right)^{2}}{E E^{\prime}}, \\
p_{\perp}^{2} \simeq-\frac{E^{\prime}}{\omega} k^{2} .
\end{gathered}
$$

Changing variables to integrate over $k^{2}$ we find

$$
d \sigma\left(e^{-} f \rightarrow e^{-} X\right)=\frac{\alpha}{2 \pi} \int d E^{\prime} \frac{\left(E^{2}+E^{\prime 2}\right)}{\omega E^{2}} \frac{d k^{2}}{\left(-k^{2}\right)} d \sigma(\gamma f \rightarrow X) .
$$

The leading term comes from the fact that the smallest $k^{2}$ is proportional to $m_{e}^{2}$ :

$$
-\int \frac{d k^{2}}{k^{2}}=\log \frac{4 E E^{\prime}}{m_{e}^{2} \omega^{2}} \simeq 2 \log \frac{E}{m_{e}}
$$

We present the final answer in the form

$$
\sigma\left(e^{-} f \rightarrow f X\right)=\left.\int d x f_{\gamma}(x) \sigma(\gamma f \rightarrow X)\right|_{\hat{s}=x s},
$$

where $\hat{s}=\left(k+p_{f}\right)^{2}$. We conclude that the leading contribution to the photon flux is

$$
f_{\gamma}(x)=\frac{\alpha}{\pi} \frac{\left[1+(1-x)^{2}\right]}{x} \log \frac{E}{m_{e}} .
$$

The term proportional to $1 / x$ derives from photons with spins aligned with the spin of the incoming electron, while the term proportional to $(1-x)^{2} / x$ provides the 


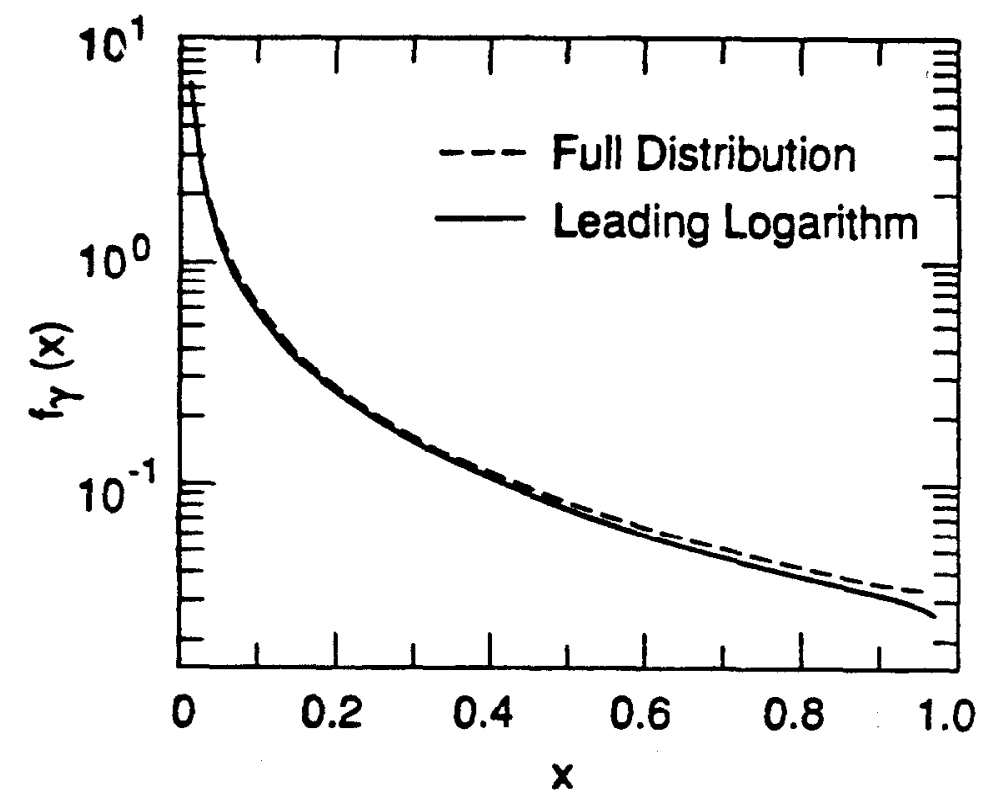

Figure 3.3 The effective flux of photons in an electron with energy $1 \mathrm{TeV}$ as a function of the momentum fraction $x$ : the full expression, Eq. (3.6), compared to the leading-logarithm approximation, Eq. (3.5).

distribution of anti-aligned photons. At an energy of $E=1 \mathrm{TeV}$ the logarithmic enhancement is $\log \frac{E}{m_{e}} \simeq 14.5$. The full expression is ${ }^{21}$

$$
\begin{aligned}
f_{\gamma}(x) & =\frac{\alpha}{\pi}\left\{\frac{\left[1+(1-x)^{2}\right]}{x}\left(\log \frac{E}{m_{e}}-\frac{1}{2}\right)\right. \\
& \left.+\frac{x}{2}\left(\log \frac{2(1-x)}{x}+1\right)+\frac{(2-x)^{2}}{2 x} \log \frac{2(1-x)}{2-x}\right\}
\end{aligned}
$$

The full photon flux compared to the "leading-log" distribution is shown in Figure 3.3. We see that at these energies the leading term approximates the full distribution to high accuracy. 


\subsection{Two-Photon Production of Top Quarks}

In the previous section we derived the effective-photon approximation for a process involving one exchanged photon. To treat photon-photon collisions we need to fold in another factor of the photon flux. Accordingly, the cross section for two-photon production of top quark pairs is

$$
\sigma\left(e^{+} e^{-} \rightarrow e^{+} e^{-} t \bar{t}\right)=\int d x_{1} d x_{2} f_{\gamma}\left(x_{1}\right) f_{\gamma}\left(x_{2}\right) \sigma(\gamma \gamma \rightarrow t \bar{t})
$$

where $\sigma(\gamma \gamma \rightarrow t \bar{t})$ is evaluated at a center-of-mass energy squared, $\hat{s}=x_{1} x_{2} s$ and $f_{\gamma}(x)$ is given by Eq. (3.6).

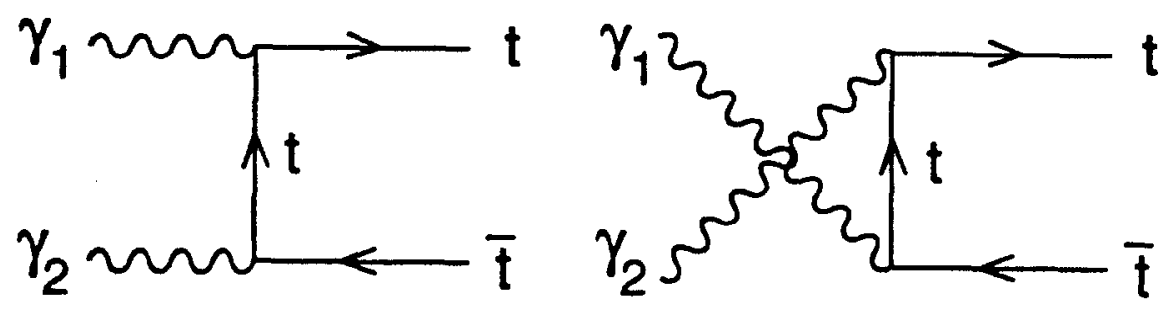

Figure 3.4 The Feynman diagrams for $\gamma \gamma \rightarrow t \bar{t}$.

The two-photon process proceeds through the two diagrams in Figure 3.4. The cross section is (for unpolarized photons)

$$
\sigma(\gamma \gamma \rightarrow t \bar{t})=4 \pi N_{c} Q_{t}^{4} \alpha^{2} \frac{\beta_{t}}{\hat{s}}\left[\frac{\mathcal{L}_{t}}{\beta_{t}}\left(1+4 \Delta_{t}-8 \Delta_{t}^{2}\right)-1-4 \Delta_{t}\right],
$$

with the dependence on the top-quark mass entering through

$$
\begin{aligned}
\Delta_{t} & =\frac{m_{t}^{2}}{\hat{s}}, \\
\beta_{t} & =\sqrt{1-4 \Delta_{t}},
\end{aligned}
$$

and 


$$
\mathcal{L}_{t}=\log \frac{1+\beta_{t}}{1-\beta_{t}}
$$

In Figure 3.5 the full cross section at $\sqrt{s}=2 \mathrm{TeV}$ is plotted for various values of $m_{t}$. We see that for $m_{t} \lesssim 100 \mathrm{GeV}$ the two-photon cross section is comparable to that from annihilation through a photon or $Z^{0}$.

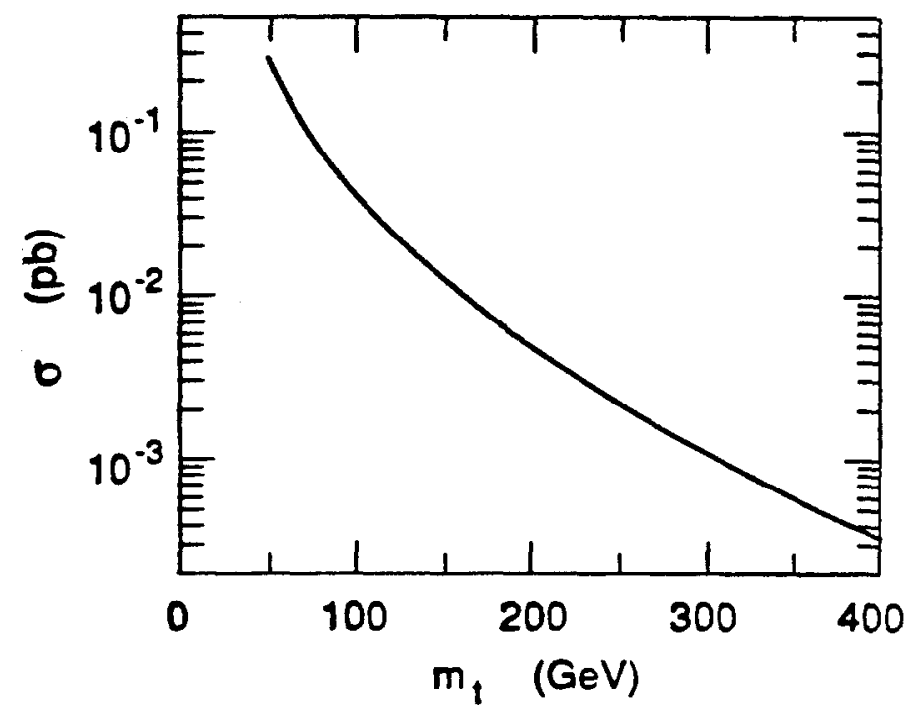

Figure 3.5 The cross section for two-photon production of top quarks, $e^{+} e^{-} \rightarrow \gamma \gamma \rightarrow t \bar{t}$, at $\sqrt{s}=2 \mathrm{TeV}$ in the effective photon approximation as a function of the top quark mass.

\subsection{THE EFFECTIVE-W APPROXIMATION}

In this section we derive the analogue for massive vector bosons of the effectivephoton approximation, in order to use this technique to calculate top quark production from the fusion of $W$ 's and $Z$ 's. The effective- $W$ approximation has been discussed extensively in the literature. ${ }^{22}$ It has been used to calculate production of very heavy Higgs particles and heavy fermion pairs arising from $W$-boson fusion, both in the context of hadron-hadron collisions and electron-positron collisions. ${ }^{23}$

There are several immediate differences between processes involving photons 
and those involving $W^{ \pm}$or $Z^{0}$. The $W^{ \pm}$and $Z^{0}$ have both vector and axialvector couplings to fermions. As we will see shortly, interference between these coupling will cause the bosons of helicity +1 to have different distributions from those with helicity -1 . Since the $W^{ \pm}$and $Z^{0}$ are massive, processes involving these particles are suppressed until very high energies are obtained. If a process is to be well-described by the effective-boson approximation, the energy that the virtual boson carries must be significantly larger than its mass. This creates a threshold below which the effective-boson approximation is no longer applicable. For example, we do not expect the effective-boson approximation to be applicable to the production of light fermion pairs, since these are produced most copiously at energies much less than $M_{z}$ and $M_{W}$. Finally, the $W^{ \pm}$and $Z^{0}$, being massive, are allowed to have longitudinal polarization states. Longitudinal polarizations couple to the mass of fermions and thus become important in heavy fermion production, whereas in photon interactions the longitudinal contributions are suppressed due to the Ward identity.

These distinctions duly noted, the derivation of the effective- $W$ approximation proceeds in a very similar fashion to our previous derivation of the effective-photon approximation. We will present the derivation in detail because there has been some controversy in the literature and because the cross sections for heavy fermion production from vector-boson fusion depend strongly on the parton distributions used. We will follow closely the treatment of Dawson, Ref. 22 . 


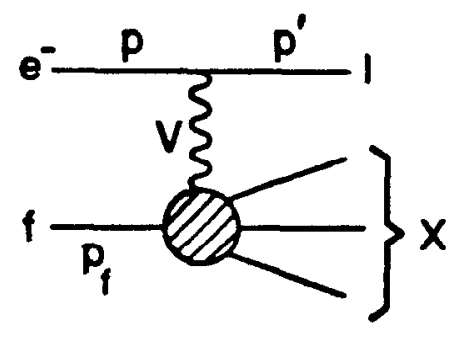

Figure 3.6 The production of an arbitrary final state $\mathrm{X}$ via exchange of a massive vector boson $\mathrm{V}$ between an electron and an arbitrary initial particle $f: e^{-} f \rightarrow l X, l$ is either an electron or a neutrino depending on whether the $V$ is a $Z^{0}$ or a $W^{-}$.

For generality, we study processes involving a massive vector boson, $V$, which may be either charged or neutral. We allow $V$ to have arbitrary vector and axialvector couplings to the electron, $g_{v}$ and $g_{a}$, respectively. Consider the process depicted in Figure 3.6. The amplitude for the process is

$$
\mathcal{M}=\bar{u}\left(p^{\prime}\right)(-i) \gamma^{\mu}\left(g_{v}+g_{a} \gamma^{5}\right) u(p) \frac{\left(-i g_{\mu \nu}\right)}{\left(k^{2}-M_{V}^{2}\right)} \mathcal{A}^{\nu}
$$

where as before $\mathcal{A}^{\nu}$ describes the three-point coupling: $V^{\nu}+f \rightarrow X$. For $W^{ \pm}$and $Z^{0}$ bosons the couplings are

$$
W^{ \pm}: \quad g_{v}=-g_{a}=\frac{g}{2 \sqrt{2}}
$$

and

$$
\begin{aligned}
Z^{0}: \quad g_{v} & =\frac{g}{\cos \theta_{w}}\left(-\frac{1}{4}+\sin ^{2} \theta_{w}\right) \\
g_{a} & =\frac{g}{4 \cos \theta_{w}}
\end{aligned}
$$


Squaring the amplitude and summing over spins yields

$$
\begin{aligned}
|\overline{\mathcal{M}}|^{2} \equiv \frac{1}{2} \sum_{\text {spins }}|\mathcal{M}|^{2} & =\frac{2}{\left(k^{2}-M_{V}^{2}\right)^{2}}\left\{\left(g_{v}^{2}+g_{a}^{2}\right)\left[p^{\mu} p^{\prime \alpha}+p^{\alpha} p^{\prime \mu}-p \cdot p^{\prime} g^{\mu \alpha}\right]\right. \\
& \left.+2 i g_{v} g_{a} \epsilon^{\mu \alpha \rho \sigma} p_{\rho} p_{\sigma}^{\prime}\right\} g_{\mu \nu} g_{\alpha \beta} \mathcal{A}^{\nu} \mathcal{A}^{\dagger \beta}
\end{aligned}
$$

We now decompose the propagator into a sum over polarization vectors. We choose unitary gauge and substitute

$$
-g_{\mu \nu}+\frac{k_{\mu} k_{\nu}}{k^{2}}=\sum_{\lambda} \epsilon_{\mu}(\lambda) \epsilon_{\nu}^{*}(\lambda)
$$

We choose the helicity basis for the polarization vectors: $\lambda$ runs over $\lambda= \pm 1,0$. The explicit polarization vectors we will use are:

$$
\begin{aligned}
& \epsilon_{ \pm}^{\mu}=\frac{-1}{\sqrt{2}}(0, \pm 1, i, 0), \\
& \epsilon_{0}^{\mu}=\frac{1}{\sqrt{-k^{2}}}(|\mathbf{k}|, 0,0, \omega),
\end{aligned}
$$

where $\hat{\mathbf{k}}$ defines the $\mathrm{z}$-axis. Note that since $k$ is a space-like vector $\left(k^{2}<0\right), \epsilon_{0}$ must be time-like, if it is to be orthogonal to $k$. Thus $\epsilon_{0} \cdot \epsilon_{0}^{*}=+1$, whereas $\epsilon_{ \pm} \cdot \epsilon_{ \pm}^{*}=-1$.

Inserting Eq. (3.9) into Eq. (3.8) yields

$$
\begin{aligned}
|\overline{\mathcal{M}}|^{2}=\frac{2}{\left(k^{2}-M_{v}^{2}\right)^{2}} \sum_{i j}\{ & \left(g_{v}^{2}+g_{a}^{2}\right)\left[\left(p \cdot \epsilon_{i}^{*}\right)\left(p^{\prime} \cdot \epsilon_{j}\right)+\left(p \cdot \epsilon_{j}\right)\left(p^{\prime} \cdot \epsilon_{i}^{*}\right)-\left(p \cdot p^{\prime}\right)\left(\epsilon_{i}^{*} \cdot \epsilon_{j}\right)\right] \\
& \left.+2 i g_{v} g_{a} \epsilon^{\mu \alpha \rho \sigma} \epsilon_{i \mu}^{*} \epsilon_{j \alpha} p_{\rho} p_{\sigma}^{\prime}\right\}\left(\mathcal{A} \cdot \epsilon_{i}\right)\left(\mathcal{A}^{\dagger} \cdot \epsilon_{j}^{*}\right) .
\end{aligned}
$$

In order for the derivation to proceed it is necessary that all the interference terms between different polarizations vanish, ${ }^{24}$ either identically or after integration over the azimuthal angle of $p^{\prime}$. This condition is indeed satisfied, as we will now see. 
To facilitate the argument define explicit components for $\mathbf{p}$ and $\mathbf{p}^{\prime}$. Momentum conservation requires that the components of $\mathbf{p}$ and $\mathbf{p}^{\prime}$ perpendicular to $\mathbf{k}$ be equal and opposite. With $\hat{\mathrm{k}}$ defining the $z$-axis we write:

$$
\begin{aligned}
\mathbf{p} & =\left(p_{\perp} \cos \phi, p_{\perp} \sin \phi, p_{3}\right), \\
\mathbf{p}^{\prime} & =\left(-p_{\perp} \cos \phi,-p_{\perp} \sin \phi, p_{3}^{\prime}\right) .
\end{aligned}
$$

Let us analyze each of the terms in Eq. (3.11) separately, focussing on the case $i \neq j$ in the dual polarization sum. When $i= \pm 1$ and $j=0$ the first two terms are linear combinations of $\sin \phi$ and $\cos \phi$ and so vanish upon the $\phi$ integration. When $i=+1$ and $j=-1$ these terms yield $p_{\perp}^{2}\left(\cos ^{2} \phi-\sin ^{2} \phi\right)$, which also integrates to zero. The third term, being proportional to $\epsilon_{i}^{*} \cdot \epsilon_{j}$, is automatically diagonal, since the different polarization vectors are orthogonal. Now examine the piece containing $\epsilon^{\mu \alpha \rho \sigma}$. The antisymmetry of the $\epsilon$ symbol causes this term to vanish when $i=+1$ and $j=-1$, since $\epsilon_{+}^{*}=-\epsilon_{-}$. When one $\epsilon$ is transverse and the other is longitudinal each term in the Lorentz sum is forced to have exactly one power of either $\sin \phi$ or $\cos \phi$, again yielding zero under the $\phi$ integral. We conclude that, as claimed, the interference terms do not contribute.

Discarding the off-diagonal terms in Eq. (3.11), and inserting the explicit polarization vectors from Eq. (3.10), we find

$$
\begin{aligned}
|\overline{\mathcal{M}}|^{2}=\frac{2}{\left(k^{2}-M_{V}^{2}\right)^{2}}\left\{\sum_{\lambda= \pm 1}\right. & {\left[\left(g_{v}^{2}+g_{a}^{2}\right)\left(p_{\perp}^{2}-\frac{k^{2}}{2}\right)-2 \lambda g_{v} g_{a}\left(E p_{3}^{\prime}-E^{\prime} p_{3}\right)\right]|\mathcal{A} \cdot \epsilon(\lambda)|^{2} } \\
& \left.+\left(g_{v}^{2}+g_{a}^{2}\right)\left[2\left(p \cdot \epsilon_{0}\right)^{2}+\frac{1}{2} k^{2}\right]\left|\mathcal{A} \cdot \epsilon_{0}\right|^{2}\right\}
\end{aligned}
$$

Note that the term proportional to $g_{v} g_{a}$ changes sign depending on the helicity 
of the virtual boson, resulting in different distributions for the two transverse polarizations. 25

We can now pass to the cross section:

$$
d \sigma\left(e^{-} f \rightarrow l X\right)=\frac{1}{2(2 \pi)^{3}} \int \frac{d^{3} p^{\prime}}{E^{\prime}}|\overline{\mathcal{M}}|^{2} d \widetilde{\Gamma}
$$

where $d \widetilde{\Gamma}$ is defined by Eq. (3.2). The integration over $\phi$ gives a factor of $2 \pi$ and the cross section becomes

$$
d \sigma\left(e^{-} f \rightarrow e-(\nu) X\right)=\frac{1}{2(2 \pi)^{2}} \int E^{\prime} d E^{\prime} d \cos \theta|\overline{\mathcal{M}}|^{2} d \widetilde{\Gamma}
$$

Up to this point we have made no approximations; Eq. (3.13) is exact, with $|\overline{\mathcal{M}}|^{2}$ given by Eq. (3.12). To implement the effective-boson approximation we assume that the amplitudes $|\mathcal{A} \cdot \epsilon(\lambda)|^{2}$ are slowly varying with respect to the rest of the integrand in Eq.(3.13) so that we can take them to their values at $\theta=0$ and remove them from the integral over $\cos \theta$. The cross section for the sub-process, $V+f \rightarrow X$ is given by $|\mathcal{A} \cdot \epsilon(\lambda)|^{2}$, multiplied by the appropriate phase-space factor. Since the transverse polarization vectors have straightforward limits at $\theta=0$ we write

$$
\lim _{\theta \rightarrow 0}\left|\mathcal{A} \cdot \epsilon_{ \pm}\right|^{2} d \widetilde{\Gamma}=\frac{\omega}{E} d \sigma\left(V_{\lambda= \pm 1}+f \rightarrow X\right)
$$

The corresponding limit for the longitudinal amplitude is slightly more subtle. The longitudinal polarization vector in Eq. (3.10) contains a factor of $1 / \sqrt{-k^{2}}$, which diverges in the forward direction. So in order to make our continuation to 
the forward direction we define a "physical" longitudinal polarization vector:

$$
\epsilon_{\mathrm{phys}}^{\mu}=\frac{k^{\mu}}{M_{v}} .
$$

We then write

$$
\lim _{\theta \rightarrow 0}\left|\mathcal{A} \cdot \epsilon_{0}\right|^{2} d \widetilde{\Gamma}=\frac{\omega}{E} \frac{M_{v}^{2}}{\left(-k^{2}\right)} d \sigma\left(V_{\lambda=0}+f \rightarrow X\right)
$$

The factor of $M_{v}^{2} /\left(-k^{2}\right)$ results from the conversion from the "virtual" polarization vector to the "physical" one, and the sub-process cross section is evaluated using $\epsilon_{\text {phys }}$.

We define the effective boson distributions, $f_{\lambda}$ by

$$
d \sigma\left(e^{-} f \rightarrow l X\right)=\left.\sum_{\lambda} \int d x f_{\lambda}(x) d \sigma\left(V_{\lambda}+f \rightarrow X\right)\right|_{\hat{s}=x s} .
$$

Comparing Eq. (3.13) and Eq. (3.15) and using Eq. (3.12) for $|\mathcal{M}|^{2}$, we can read off the distribution functions:

$$
f_{ \pm}(x)=\frac{E^{\prime} \omega}{4 \pi^{2}} \int \frac{d \cos \theta}{\left(k^{2}-M_{v}^{2}\right)^{2}}\left[\left(g_{v}^{2}+g_{a}^{2}\right)\left(p_{\perp}^{2}-\frac{1}{2} k^{2}\right) \pm 2 g_{v} g_{a}\left(E^{\prime} p_{3}-E p_{3}^{\prime}\right)\right]
$$

and

$$
f_{0}(x)=\frac{E^{\prime} \omega}{4 \pi^{2}} \int \frac{d \cos \theta}{\left(k^{2}-M_{v}^{2}\right)^{2}} \frac{M_{v}^{2}}{\left(-k^{2}\right)}\left(g_{v}^{2}+g_{a}^{2}\right)\left[\left(p \cdot \epsilon_{0}\right)^{2}+\frac{1}{2} k^{2}\right] .
$$

It is convenient to define linear combinations of the transverse distributions:

$$
F_{ \pm}(x) \equiv f_{+}(x) \pm f_{-}(x)
$$

Before performing the integrals in Eqs. (3.16) and (3.17) we need to do some kinematics, expressing the relevant quantities in terms of $s$ and $k^{2}$. The quantities 
we need are

$$
\begin{gathered}
p_{\perp}^{2}=\frac{E^{2} E^{\prime 2} \sin ^{2} \theta}{|\mathbf{k}|^{2}}=\frac{-k^{2}}{4|\mathbf{k}|^{2}}\left[s(1-x)+k^{2}\right], \\
E^{\prime} p_{3}-E p_{3}^{\prime}=\frac{-k^{2}}{2|\mathbf{k}|}\left(E+E^{\prime}\right), \\
\left(p \cdot \epsilon_{0}\right)^{2}=\frac{-k^{2} s}{16|\mathbf{k}|^{2}}(2-x)^{2}
\end{gathered}
$$

with $|k|^{2}=\omega^{2}-k^{2}$. With these substitutions we have

$$
\begin{aligned}
& F_{+}(x)=\frac{\left(g_{v}^{2}+g_{a}^{2}\right)}{16 \pi^{2}} x \int \frac{d k^{2}\left(-k^{2}\right)}{\left(\omega^{2}-k^{2}\right)\left(k^{2}-M_{v}^{2}\right)^{2}}\left[s(1-x)+2 \omega^{2}-k^{2}\right], \\
& F_{-}(x)=\frac{g_{a} g_{v}}{4 \pi^{2}} E(2-x) \int \frac{d k^{2}\left(-k^{2}\right)}{\sqrt{\omega^{2}-k^{2}}} \frac{1}{\left(k^{2}-M_{v}^{2}\right)^{2}}, \\
& f_{0}(x)=\frac{\left(g_{v}^{2}+g_{a}^{2}\right)}{64 \pi^{2}} x M_{v}^{2} \int \frac{d k^{2}}{\left(\omega^{2}-k^{2}\right)\left(k^{2}-M_{v}^{2}\right)^{2}}\left[s(2-x)^{2}-4\left(\omega^{2}-k^{2}\right)\right] .
\end{aligned}
$$

The integrals are straightforward to perform; the results are:

$$
\begin{gathered}
F_{+}(x)=\frac{\left(g_{v}^{2}+g_{a}^{2}\right)}{16 \pi^{2}} x\left\{\left[1+\frac{(2-x)^{2}}{x^{2} \eta^{4}}\right] \log \left(1+\frac{1-x}{\Delta}\right)\right. \\
\left.-\frac{2(2-x)^{2}}{x^{2} \eta^{4}} \log \left(\frac{2-x}{x}\right)+\frac{4(1-x)\left(x^{2} / 2+1-x+\Delta\right)}{x^{2} \eta^{2}(1-x+\Delta)}\right\}, \\
F_{-}(x)=\frac{g_{a} g_{v}}{8 \pi^{2}} \frac{(2-x)}{x^{2} \eta^{3}}\left\{\left(x^{2}-2 \Delta\right) \log \left[\frac{(2-x-x \eta)}{(2-x+x \eta)} \frac{(1+\eta)}{(1-\eta)}\right]\right. \\
\left.\quad+\frac{\Delta \eta x(2-x)}{1-x+\Delta}+x^{2} \eta\right\}, \\
f_{0}(x)=\frac{\left(g_{v}^{2}+g_{a}^{2}\right)}{4 \pi^{2} x \eta^{2}}\left\{\frac{\Delta}{\eta^{2} x^{2}}(2-x)^{2} \log \left[\frac{(2-x)^{2}}{x^{2}} \frac{\Delta}{s(1-x)+\Delta}\right]+1-x\right\},
\end{gathered}
$$


with the definitions:

$$
\begin{aligned}
\Delta & =\frac{M_{v}^{2}}{s} \\
\eta & =\sqrt{1-\frac{4 \Delta}{x^{2}}} .
\end{aligned}
$$

Although Eqs. (3.19) - (3.21) appear singular as $\eta \rightarrow 0$, they are in fact wellbehaved, as they must be, since Eqs. (3.18) are clearly smooth as $\omega \rightarrow M_{V}$.

The leading terms in the distributions are obtained by taking $\Delta \ll x^{2}$ (which of course forces $\Delta \ll 1$ ). The distributions in this limit become

$$
\begin{aligned}
& F_{+}(x)=\frac{\left(g_{v}^{2}+g_{a}^{2}\right)}{8 \pi^{2}} \frac{\left[1+(1-x)^{2}\right]}{x} \log \left(\frac{1}{\Delta}\right), \\
& F_{-}(x)=\frac{g_{v} g_{a}}{4 \pi^{2}}(2-x) \log \left(\frac{1}{\Delta}\right) \\
& f_{0}(x)=\frac{\left(g_{v}^{2}+g_{a}^{2}\right)}{4 \pi^{2}} \frac{1-x}{x}
\end{aligned}
$$

The averaged transverse distribution, $F_{+}(x)$, is the analogue of the effectivephoton distribution, derived in Section 1. Whereas the photon flux is enhanced by a factor of $\log \left(s / m_{e}^{2}\right)$, the transverse states of a massive vector boson, $V$, are enhanced by a factor of $\log \left(s / M_{v}^{2}\right)$, a much weaker enhancement. The boson mass takes the place of $m_{e}$ because it is $M_{V}$ that prevents the boson propagator from hitting the pole. The relative enhancements can be seen in Figure 3.7 in which we plot the fluxes in the leading log approximation for the case of a $W^{-}$being emitted from an electron; the photon flux is presented for comparison. We should note that approximating $F_{+}(x)$ by its leading term can lead to a gross overestimate of the flux, since the term proportional to $\log (1 / x)$ may cancel destructively against the leading term, ${ }^{26}$ especially at small $x$. The parity-violating distribution, $F_{-}(x)$, 
which is not present in the effective-photon distribution, causes the two transverse helicities to have distinct fluxes. For $Z^{0}$ bosons, for which the vector coupling to electrons is very small, $F_{-}(x)$ is negligible and the two helicities, \pm 1 , have approximately the same flux.

For $W^{ \pm}$bosons, the parity-violating term changes sign depending on the charge of the $W$, as required by $C P$ invariance. Since $W$ bosons have $V-A$ couplings, $F_{-}(x)$ is negative, causing left-handed $W^{-9}$ s to be enhanced over right-handed ones, and vice versa for $W^{+}$'s. To see this in more detail, assume the neutrino in Figure 3.6 to be emitted at a small angle $\theta$ from the incoming electron. Defining the polarization vectors as in Eq. (3.10) and using explicit left-handed spinors, we can readily compute

$$
\begin{aligned}
\bar{u}_{L}(p) \gamma^{\mu} u_{L}\left(p^{\prime}\right) \epsilon_{\mu}^{+} & \sim \frac{(1-x)}{x} \theta \\
\bar{u}_{L}(p) \gamma^{\mu} u_{L}\left(p^{\prime}\right) \epsilon_{\mu}^{-} & \sim \frac{1}{x} \theta
\end{aligned}
$$

We see that for $x$ close to 1 , when the $W$ carries most of the momentum, the left-handed polarization dominates; while for small $x$ the two helicities are equally likely. This behavior is manifest in the leading log distributions. If we write the helicity distributions for a $W^{-}$we find:

$$
\begin{aligned}
& f_{-}(x)=\frac{g^{2}}{32 \pi^{2}} \frac{1}{x} \log \left(\frac{1}{\Delta}\right) \\
& f_{+}(x)=\frac{g^{2}}{32 \pi^{2}} \frac{(1-x)^{2}}{x} \log \left(\frac{1}{\Delta}\right)
\end{aligned}
$$

The distribution of longitudinal bosons does not exhibit the logarithmic enhancement of the transverse modes. Instead, the longitudinal mode is enhanced by a factor of $s / M_{v}^{2}$, which we have absorbed into our definition of the longitudinal 


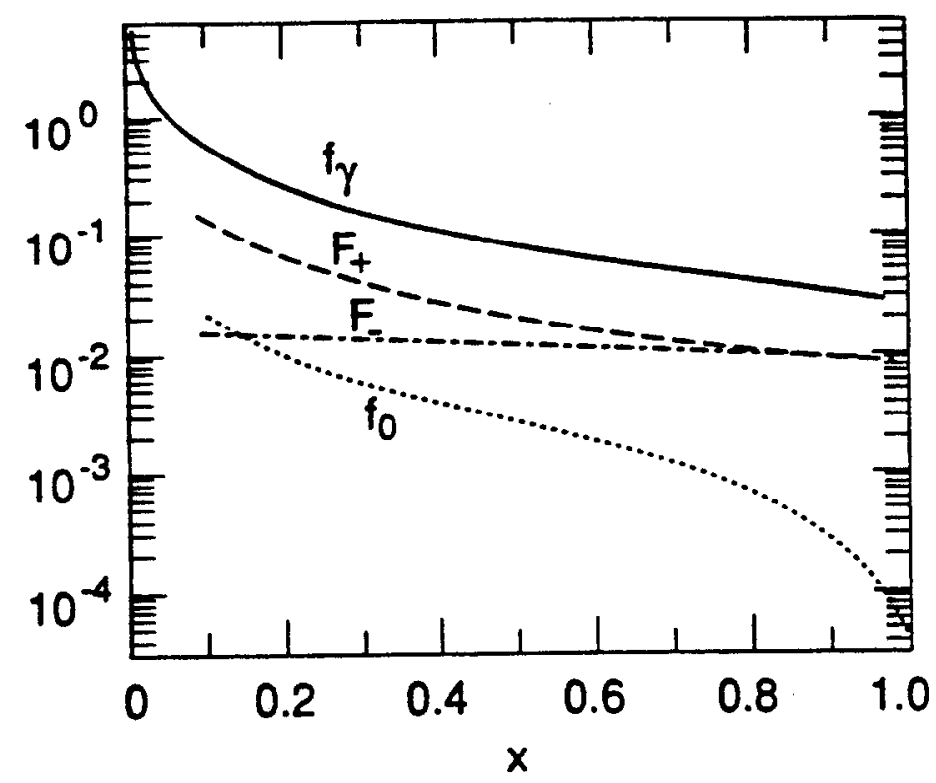

Figure 3.7 The leading logarithm approximation to effective distribution for $W$ bosons in an electron [Eqs. (3.22)-(3.24)] with beam energy $1 \mathrm{TeV}$ as a function of the momentum fraction $x$. The effective photon distribution is shown for comparison.

polarization vector. The longitudinal and transverse fluxes depend differently on $M_{V}^{2} / s$ as a result of their different kinematics: emission of a longitudinal boson is allowed in the full forward direction, while the emission of a transverse boson is forbidden by angular momentum conservation. We also note that for the longitudinal distribution the leading term accurately approximates the full distribution since the next order term is suppressed by a factor of $M_{\nu}^{2} / s$.

The distributions derived here agree in leading approximation with those in the literature. ${ }^{22}$ However, the non-leading terms differ between authors depending on the exact definitions of the distribution functions and the extent to which higher order terms in $M_{v}^{2} / s$ are retained. In situations in which the effectiveboson approximation is accurate these differences in the non-leading terms are not 
important numerically. For definiteness we will use the distribution functions of Eqs. (3.19) - (3.21). They are displayed in Figure 3.8 for a beam energy of $1 \mathrm{TeV}$, along with the leading approximations for comparison. We see that the leading approximation to the longitudinal distribution is quite accurate, whereas in the transverse case the leading-log distribution differs from the full distribution by as much as a factor of 10 .

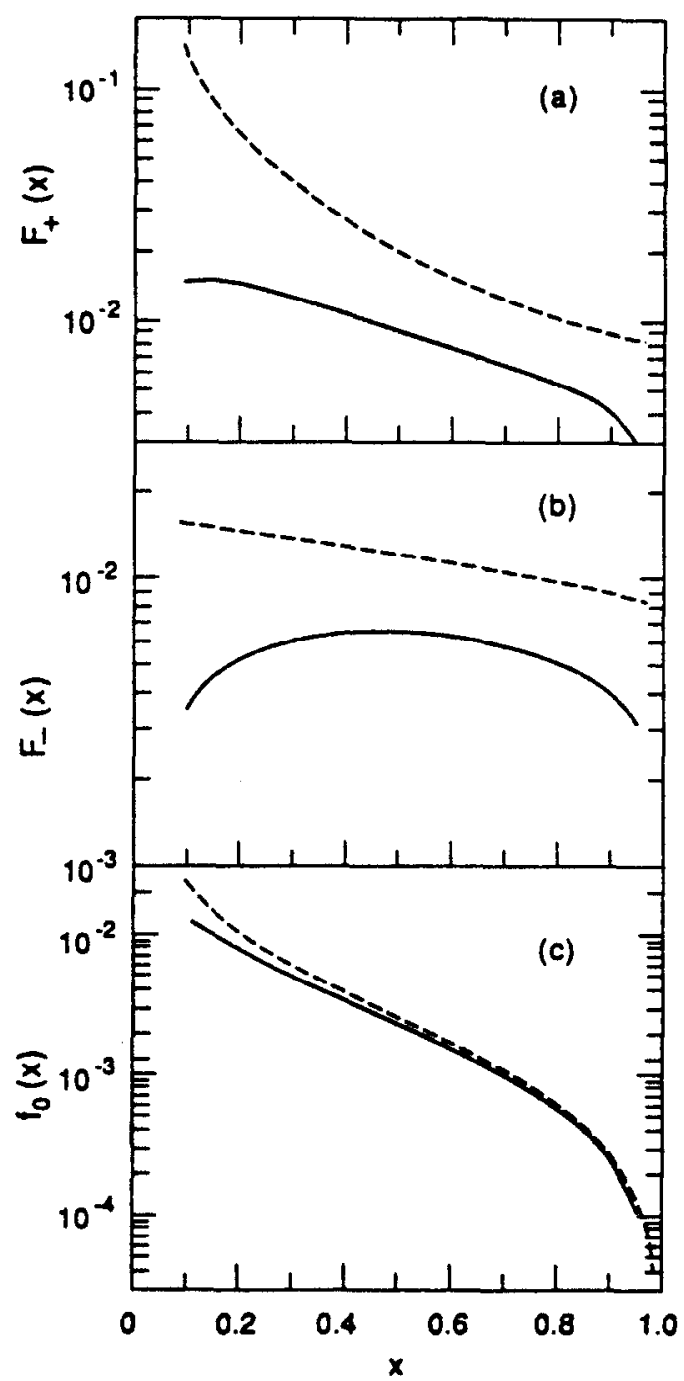

Figure 3.8 Comparison of the full expression for the $W$ fluxes in an electron (solid lines) at energy $1 \mathrm{TeV}$ with the leading logarithm approximations (dashed lines) as a function of the momentum fraction $x$ : a) Sum of the two transverse distribution, $F_{+}(x)$; b) Difference of the two transverse distribution, $F_{+}(x)$; c) The longitudinal distribution, $f_{0}(x)$. 


\subsection{TOP-QUARK Production From $W W$ FUSION}

In this section we compute the cross section for $e^{+} e^{-} \rightarrow \nu \bar{\nu} t \bar{t}$ through $W W$ fusion. We will treat this process in the effective- $W$ approximation derived in the previous section. By employing the effective $-W$ approximation we restrict out attention to the so-called $W$-fusion diagrams, those of the form shown in Figure 3.9. We expect these diagrams to contribute the bulk of the cross sections

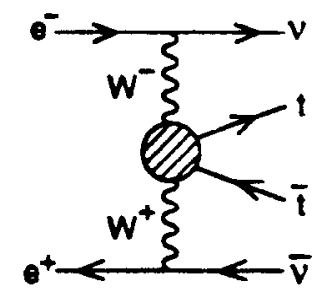

Figure 3.9 Production of top quark pairs via $W W$ fusion.

at high enough energies. The "peripheral diagrams" that we neglect are shown in Figure 3.10. The degree to which these diagrams alter the cross section is unknown in general although there have been exact calculations in some special cases. ${ }^{27}$

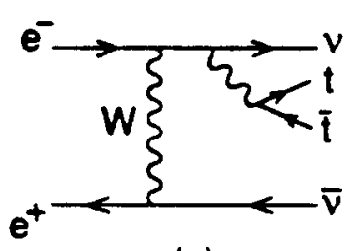

(a)

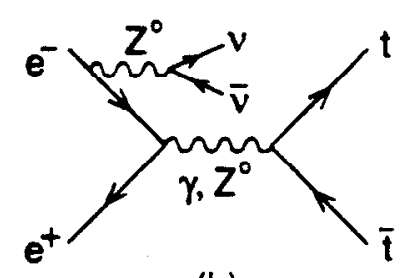

(b)

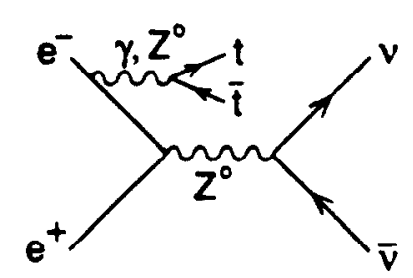

(c)

Figure 3.10 Peripheral diagrams for $e^{+} e^{-} \rightarrow \nu \bar{\nu} t \bar{t}$, neglected in the effective- $W$ approximation: a,c) the intermediate boson coupling to the $t \bar{t}$ pair can be a $Z^{0}$ emitted from any of the four lepton legs or a photon from the electron or positron; b) the $Z^{0}$ can be emitted from any of the four fermion lines. 
Since the effective- $W$ approximation yields different distributions for the three possible polarizations of the $W$ 's, we must treat the polarizations separately when computing the cross section for the subprocess $W^{+} W^{-} \rightarrow t \bar{t}$. Furthermore, the appropriate choice of basis for the polarization vectors of the $W$ 's is the helicity basis, since the effective- $W$ approximation is diagonal only in that basis. Accordingly, we write the full cross section as

$$
\begin{aligned}
\sigma\left(e^{+} e^{-} \rightarrow \nu \bar{\nu} t \bar{t}\right) & =\sum_{\lambda_{+} \lambda_{-}} \sigma\left(e^{+} e^{-} \rightarrow W_{\lambda_{+}}^{+} W_{\lambda_{-}}^{-} \rightarrow t \bar{t}\right) \\
& =\sum_{\lambda_{+} \lambda_{-}} \int d x_{+} d x_{-} f_{\lambda_{+}}\left(x_{+}\right) f_{\lambda_{-}}\left(x_{-}\right) \sigma\left(W_{\lambda_{+}}^{+} W_{\lambda_{-}}^{-} \rightarrow t \bar{t}\right),
\end{aligned}
$$

where the helicities $\lambda_{ \pm}$of the $W \pm$ each run over $1,0,-1$.

The cross section for the sub-process $W^{+} W^{-} \rightarrow t \bar{t}$ is straightforward, if tedious, to calculate. It proceeds through the diagrams in Figure 3.11 .

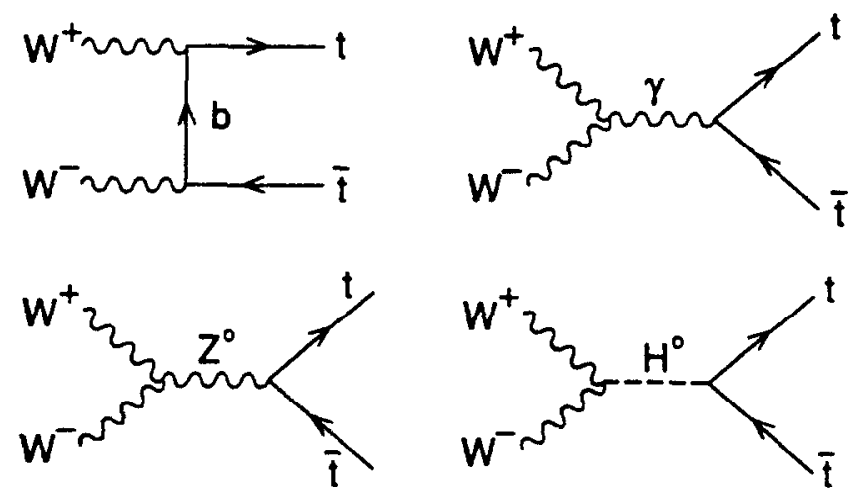

Figure 3.11 Diagrams contributing to $W^{+} W^{-} \rightarrow t \bar{t}$.

Along with the Compton-like graph, familiar from photon-photon fusion, we have the $s$-channel graphs involving the photon, $Z^{0}$, and Higgs. It is well known that the individual graphs are not well behaved at high energies and that it is only the sum of the graphs which is unitary. The cancellations between diagrams 
are especially large for processes involving longitudinal bosons, since they have a polarization vector which grows with energy: $\epsilon^{\mu} \simeq k^{\mu} / M_{w}$. For simplicity, we will present cross sections summed over the spins of the $t$ and $\bar{t}$. We note that, since the $W$ has left-handed couplings to fermions, left-handed top quarks (accompanied by right-handed anti-quarks) will dominate.

In the calculation of $W^{+} W^{-} \rightarrow t \bar{t}$ cross section we will take the momenta of the $W$ 's to be light-like: $k^{2}=0$. There are two reasons for this. First, the limit of the integration over $k^{2}$ in the effective- $W$ approximation is $k^{2}=0$; continuing $k^{2}$ to $k^{2}=M_{W}^{2}$ adds additional error at the order of $M_{w}^{2} / s$. Second, it is simpler to perform the calculation for $k^{2}=0$ than for $k^{2}=M_{W}^{2}$. For polarization vectors we take those defined in Eq. (3.10) for helicity \pm 1 , and Eq. (3.14) for the longitudinal polarization.

We begin with the cases where both the $W^{+}$and $W^{-}$are transverse: $\lambda= \pm 1$. Note that not all of these configurations are independent: $C P$ invariance requires $\sigma\left(W_{\lambda=+1}^{+} W_{\lambda=+1}^{-} \rightarrow t \bar{t}\right)=\sigma\left(W_{\lambda=-1}^{+} W_{\lambda=-1}^{-} \rightarrow t \bar{t}\right)$. The actual calculation of the cross section is routine: add the various diagrams, square the full matrix element, sum over the quark spins, and finally integrate over the phase space. The cross sections for the various helicity combinations are:

$$
\begin{aligned}
& \sigma\left(W_{\lambda=+1}^{+} W_{\lambda=-1}^{-} \rightarrow t \bar{t}\right)=\frac{\pi N_{c} \alpha^{2}}{2 s_{W}^{4}} \frac{\beta_{t}}{\hat{s}}\left\{4 \Delta_{t}^{2}\left(1-4 \Delta_{t}\right) \frac{\mathcal{L}_{t}}{\beta_{t}}+\frac{1}{3}+\frac{2}{3} \Delta_{t}-8 \Delta_{t}^{2}\right\} \\
& \sigma\left(W_{\lambda=-1}^{+} W_{\lambda=+1}^{-} \rightarrow t \bar{t}\right)=\frac{\pi N_{c} \alpha^{2}}{s_{w}^{4}} \frac{\beta_{t}}{\hat{s}}\left\{2 \frac{\mathcal{L}_{t}}{\beta_{t}}\left[1-4 \Delta_{t}+7 \Delta_{t}^{2}-4 \Delta_{t}^{3}\right]-\frac{17}{6}+\frac{19}{3} \Delta_{t}-4 \Delta_{t}^{2}\right\} .
\end{aligned}
$$




$$
\begin{aligned}
& \sigma\left(W_{\lambda=+1}^{+} W_{\lambda=+1}^{-} \rightarrow t \bar{t}\right)=\sigma\left(W_{\lambda=-1}^{+} W_{\lambda=-1}^{-} \rightarrow t \bar{t}\right) \\
& =\frac{\pi N_{c} \alpha^{2}}{2 s_{W}^{4}} \frac{\beta_{t}}{\hat{s}}\left\{4 \Delta_{t}^{2} \frac{\mathcal{L}_{t}}{\beta_{t}}\left[3-4 \Delta_{t}-X_{z}\left(1-2 \Delta_{t}\right)+X_{t}\left(1-\Delta_{t}\right)+\operatorname{Re} X_{H}\left(1-2 \Delta_{t}\right)\right]\right. \\
& +\Delta_{t}\left[4-2 X_{z}+X_{t}+2 \operatorname{Re} X_{H}-\frac{1}{3} X_{z}^{2}-\frac{2}{3} X_{z} X_{t}+\frac{1}{3} X_{t}^{2}+\left|X_{H}\right|^{2}\right] \\
& \left.\quad-2 \Delta_{t}^{2}\left[6-2 X_{z}+X_{t}+6 \operatorname{Re} X_{H}+2\left|X_{H}\right|^{2}\right]+\frac{1}{6}\left[2 X_{z}^{2}-2 X_{z} X_{t}+X_{t}^{2}\right]\right\}
\end{aligned}
$$

The quantities $\mathcal{L}_{t}, \beta_{t}$, and $\Delta_{t}$ are defined as in Eq. (3.7), while $s_{W}$ is defined as before, $s_{w}=\sin \theta_{w}$. The other quantities are

$$
\begin{aligned}
X_{z} & =\frac{M_{z}^{2}}{\hat{s}-M_{Z}^{2}}, \\
X_{t} & =4 Q_{t} s_{W}^{2} X_{z}, \\
\operatorname{Re} X_{H} & =\frac{M_{H}^{2}\left(\hat{s}-M_{H}^{2}-\Gamma_{H}^{2}\right)}{\left(\hat{s}-M_{H}^{2}\right)^{2}+M_{H}^{2} \Gamma_{H}^{2}}, \\
\left|X_{H}\right|^{2} & =\frac{M_{H}^{2}\left(M_{H}^{2}+\Gamma_{H}^{2}\right)}{\left(\hat{s}-M_{H}^{2}\right)^{2}+M_{H}^{2} \Gamma_{H}^{2}} .
\end{aligned}
$$

There are two principal factors at work in Eqs. (3.26) - (3.28). First, the $t$ and $\bar{t}$ quarks want to be left-handed and right-handed, respectively, because of the $V-A$ coupling to the $W$. Second, the helicity of the $t$ prefers to follow that of the $W^{+}$while the helicity of the $\bar{t}$ tends to follow the $W^{-}$. The only configuration in which both of these conditions can be met is when the $W^{+}$is left-handed and the $W^{-}$is right-handed; then the cross section receives the enhancement proportional to $\mathcal{L}_{t}$, as exhibited in Eq. (3.27). The other cases do not receive this enhancement: Eq. (3.26) has a constant term in place of the logarithm and Eq. (3.28) is suppressed by powers of $m_{t}^{2} / \hat{s}$ and $M_{z}^{2} / \hat{s}$. (Note that only these latter states with spin projection $s_{z}=0$ along the collision axis receive any contribution 
from the photon, $Z^{0}$, and Higgs diagrams, and so have any dependence on $M_{z}$ or $M_{H}$.)

To obtain the contribution of each of the intermediate polarizations to the full cross section for $e^{+} e^{-} \rightarrow \nu \bar{\nu} t \bar{t}$ we need to integrate over the flux distributions using Eq. (3.25). We note that the contributions from $W_{\lambda=-1}^{+} W_{\lambda=-1}^{-}$and $W_{\lambda=+1}^{+} W_{\lambda=+1}^{-}$ are equal, since the cross sections for the subprocess and the distributions are equal. The results of the numerical integration are shown in Figure 3.12. Comparing with the photon-photon results from Figure 3.5 we see that the contribution to $t \bar{t}$ production from transverse $W$ 's is three orders of magnitude smaller. This difference is due mainly to the fluxes of $W$ 's being much smaller than the photon flux, especially at small momentum fractions.

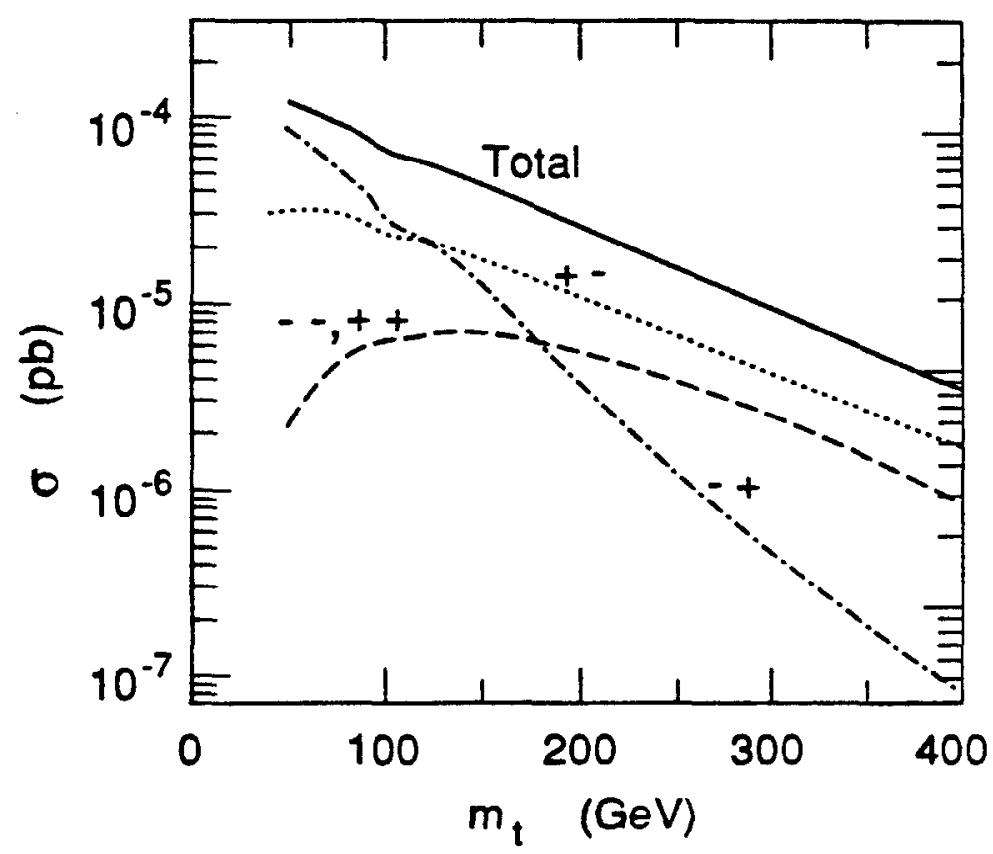

Figure 3.12 Contributions to the cross section for $e^{+} e^{-} \rightarrow \nu \bar{\nu} t \bar{t}$ at $\sqrt{s}=2 \mathrm{TeV}$ from fusion of transverse $W$ 's in the effective- $W$ approximation as a function of $m_{t}$.

Now consider the processes involving longitudinal $W$ 's. The longitudinal state 
couples differently than the transverse ones, its polarization vector being $\epsilon_{0}^{\mu}=$ $k^{\mu} / M_{W}$. Naively, this would lead to a unitarity-violating coupling proportional to $\hat{s} / M_{W}^{2}$. However, large cancellations between the various diagrams reduce this to $m_{t}^{2} / M_{W}^{2}$, at sufficiently high energies. We can explain this behavior in terms of the Higgs mechanism. It is the charged part of the original scalar doublet which is "eaten" to provide the longitudinal degree of freedom of the $W^{ \pm}$. At high cnergies $\left(\hat{s} \gg M_{W}^{2}\right)$, the electro-weak symmetry is restored and the longitudinal state couples like the scalar particle from which it came. Just like the Higgs, the longitudinal state couples to fermions via their masses. For very heavy fermions this can yield a substantial enhancement, one factor of $m_{f} / M_{W}$ in the amplitude for every longitudinal $W$ involved. We begin by including one longitudinal $W$. We can easily calculate the contributions from helicity states in which one $W$ is transverse and the other longitudinal. Just as in the completely transverse case, the relative sizes of the cross sections are determined by the helicity structure of the initial state. The process with $\lambda_{+}=-1$ and $\lambda_{-}=0$ will be enhanced over that in which $\lambda_{+}=+1$ and $\lambda_{-}=0$. Invariance under $C P$ requires equality among the cross sections for some of the initial states:

$$
\begin{aligned}
& \sigma\left(W_{\lambda=+1}^{+} W_{\lambda=0}^{-} \rightarrow t \bar{t}\right)=\sigma\left(W_{\lambda=0}^{+} W_{\lambda=-1}^{-} \rightarrow t \bar{t}\right) \\
& \sigma\left(W_{\lambda=-1}^{+} W_{\lambda=0}^{-} \rightarrow t \bar{t}\right)=\sigma\left(W_{\lambda=0}^{+} W_{\lambda=+1}^{-} \rightarrow t \bar{t}\right) .
\end{aligned}
$$

After some algebra we can write down the two independent cross sections, for a left-handed $W^{+}$and longitudinal $W^{-}$, 


$$
\begin{aligned}
\sigma\left(W_{\lambda=-1}^{+} W_{\lambda=0}^{-} \rightarrow t \bar{t}\right)= & \frac{\pi N_{c} \alpha^{2}}{s_{W}^{2}}\left(\frac{m_{t}}{M_{W}}\right)^{2} \frac{\beta_{t}}{\hat{s}} \\
& \times\left\{\frac{\mathcal{L}_{t}}{\beta_{t}}\left[\left(1-2 \Delta_{t}+2 \Delta_{t}^{2}\right)\left(1-X_{z}\right)-\Delta_{t} X_{t}\left(1-\Delta_{t}\right)\right]\right. \\
& +\frac{1}{6}\left[6-3 X_{z}+\frac{9}{2} X_{t}+X_{z}^{2}-2 X_{z} X_{t}-X_{t}^{2}\right] \\
& \left.+\Delta_{t}\left[1-X_{z}+\frac{1}{2} X_{t}\right]+\frac{1}{6 \Delta_{t}}\left[X_{z}^{2}-X_{z} X_{t}+\frac{1}{2} X_{t}^{2}\right]\right\}
\end{aligned}
$$

and for a right-handed $W^{+}$with a longitudinal $W^{-}$,

$$
\begin{aligned}
\sigma\left(W_{\lambda=+1}^{+} W_{\lambda=0}^{-} \rightarrow\right. & t \bar{t})=\frac{\pi N_{c} \alpha^{2}}{s_{W}^{2}}\left(\frac{m_{t}}{M_{W}}\right)^{2} \frac{\beta_{t}}{\hat{s}}\left\{\frac{\Delta_{t} \mathcal{L}_{t}}{\beta_{t}}\left[X_{t}+\Delta_{t}\left(2-2 X_{z}+X_{t}\right)\right]\right. \\
& +\Delta_{t}\left[1-X_{z}+\frac{1}{2} X_{t}\right]-\frac{1}{6}\left[3 X_{z}-\frac{3}{2} X_{t}+X_{z}^{2}+2 X_{z} X_{t}-X_{t}^{2}\right] \\
& \left.+\frac{1}{6 \Delta_{t}}\left[X_{z}^{2}-X_{z} X_{t}+\frac{1}{2} X_{t}^{2}\right]\right\}
\end{aligned}
$$

We see that, as advertised, the leading terms in the cross sections at high energies are proportional to $\left(m_{t} / M_{W}\right)^{2}$. The cross section for a left-handed $W^{+}$, Eq. (3.30), contains the logarithmic enhancement proportional to $\mathcal{L}_{t}$. The righthanded cross section, Eq. (3.31), is suppressed by a power of $\Delta_{t}$ relative to the left-handed process. Neither process depends on the Higgs, since coupling of a transverse $W$ and a longitudinal $W$ to the scalar Higgs is forbidden.

To obtain the contribution of these processes to $e^{+} e^{-} \rightarrow \nu \bar{\nu} t \bar{t}$ we again need to fold in the effective- $W$ distributions from Eqs. (3.19)-(3.21) and integrate over the momentum fractions of the two $W$ 's. To get the full contribution we multiply by a factor of two to account for the cross sections in which the $W^{+}$is longitudinal and the $W^{-}$is transverse. The results are displayed in Figure 3.13. We see that the contribution from $W_{\lambda=+1}^{+} W_{\lambda=0}^{-}$dominates, as expected. The factor of $\left(m_{t} / M_{W}\right)^{2}$ 
causes the cross section to be basically flat as $m_{t}$ grows. However, it never surpasses the two-photon result.

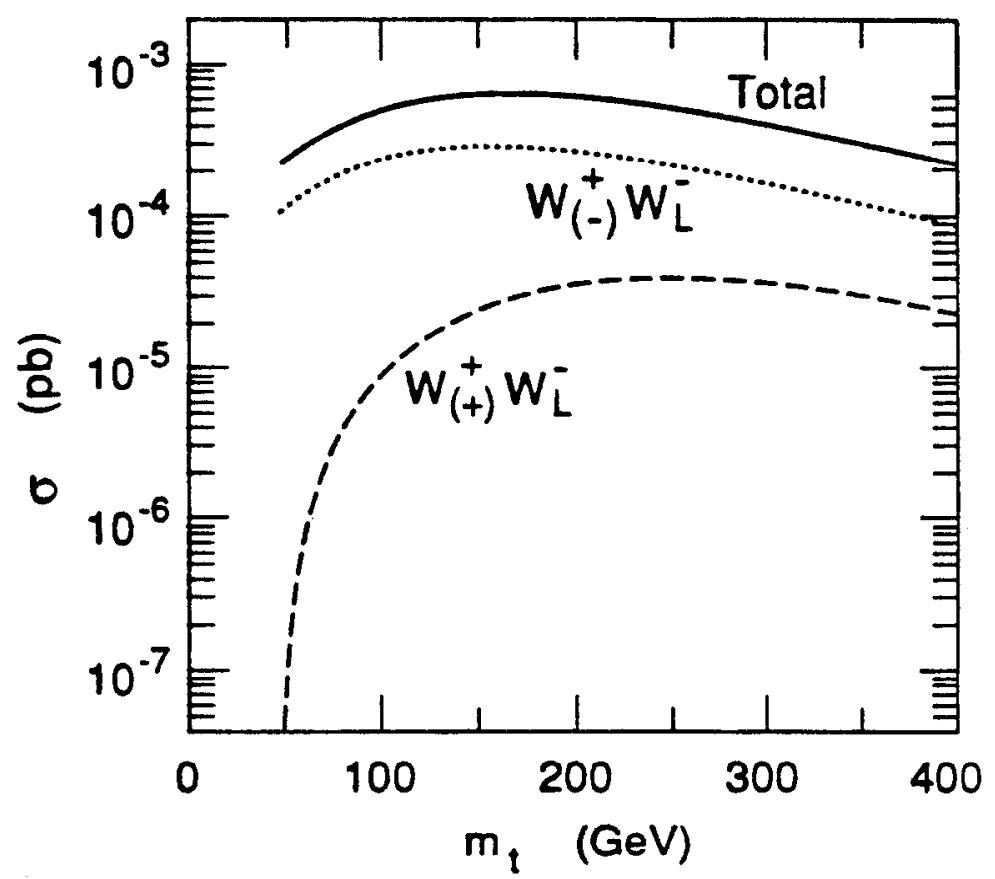

Figure 3.13 Contributions to the cross section for $e^{+} e^{-} \rightarrow \nu \bar{\nu} t \bar{t}$ at $\sqrt{s}=2 \mathrm{TeV}$ from fusion of a transverse $W$ with a longitudinal one in the effective- $W$ approximation as a function of $m_{t}$.

The remaining configuration to be considered is when both $W$ 's are longitudinally polarized. The leading contribution to this cross section is proportional to $\left(m_{t} / M_{w}\right)^{4}$. In this case the Higgs plays a critical role. The $t$-channel, photon, and $Z^{0}$ diagrams add to cancel the bulk of the unitarity-violating behavior. However, there are terms of order $m_{t} \sqrt{s} / M_{W}^{2}$ in the amplitude that remain, only to be cancelled by the Higgs contribution. At energies below the Higgs mass $\left(\sqrt{\hat{s}}<M_{H}^{2}\right)$, this cancellation will not occur. ${ }^{28}$ This will lead to an enhancement for top-quark masses less than $M_{H} / 2$.

The cross section is 


$$
\begin{aligned}
& \sigma\left(W_{\lambda=0}^{+} W_{\lambda=0}^{-} \rightarrow t \bar{t}\right)=\frac{\pi N_{c} \alpha^{2}}{8 s_{W}^{2}}\left(\frac{m_{t}}{M_{W}}\right)^{4} \frac{\beta_{t}}{\hat{s}} \\
& \quad \times\left\{\frac{4 \mathcal{L}_{t}}{\beta_{t}}\left[1+\frac{1}{2} \operatorname{Re} X_{H}+\Delta_{t}\left(2 X_{z}-X_{t}\right)\right]-4\left[1-X_{z}+\frac{1}{2} X_{t}+\operatorname{Re} X_{H}+\left|X_{H}\right|^{2}\right]\right. \\
& \left.-\frac{1}{3 \Delta_{t}}\left[3 X_{t}+X_{z}^{2}+2 X_{Z} X_{t}-X_{t}^{2}-\left|X_{H}\right|^{2}\right]+\frac{1}{3 \Delta^{2}}\left[X_{z}^{2}-2 X_{z} X_{t}+X_{t}^{2}\right]\right\}
\end{aligned}
$$

It contains the leading factor of $\left(m_{t} / M_{W}\right)^{4}$, as claimed. The strong dependence on the Higgs mass comes from the term containing $\left|X_{H}\right|^{2} / \Delta_{t}$. When the center of mass energy $\sqrt{\hat{s}}$ is substantially less than the Higgs mass, $\left|X_{H}\right|^{2} \simeq 1$. In this case the term proportional to $\left|X_{H}\right|^{2} / \Delta_{t}$ produces a term proportional to $m_{t}^{2} \hat{s} / M_{W}^{4}$. Of course as soon as $\hat{s}$ grows beyond $M_{H}^{2}$, the correct asymptotic behavior is restored.

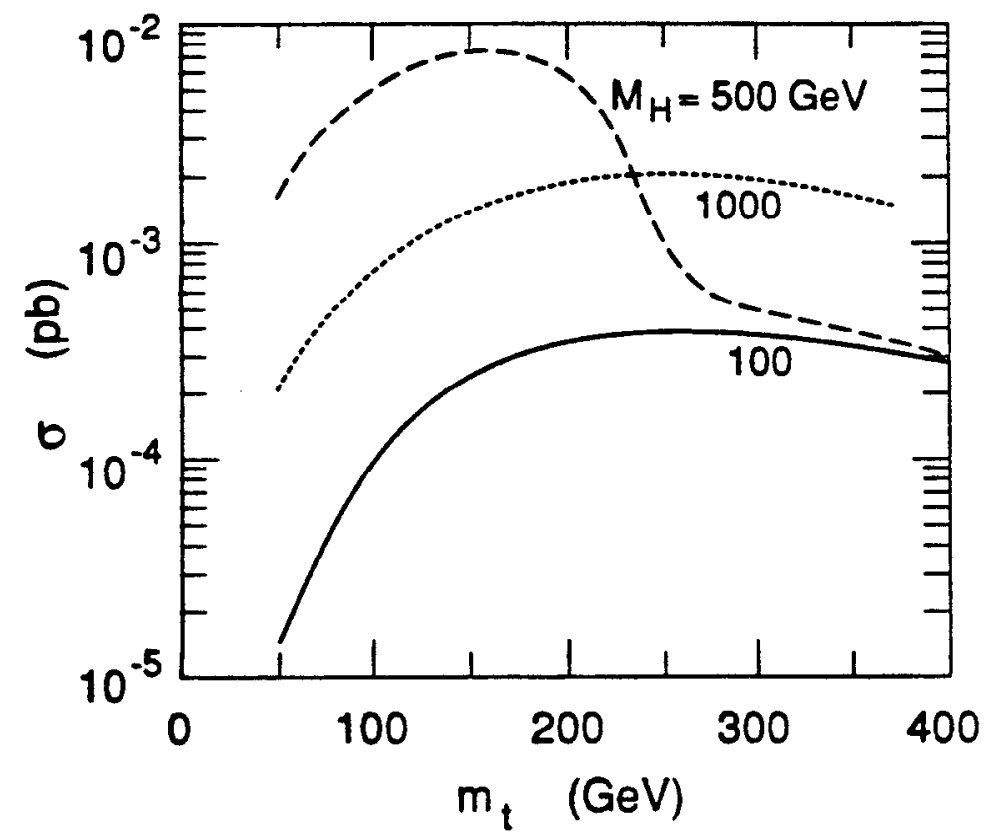

Figure 3.14 Contributions to the cross section for $e^{+} e^{-} \rightarrow \nu \bar{\nu} t \bar{t}$ at $\sqrt{s}=2 \mathrm{TeV}$ from fusion of longitudinal $W$ 's in the effective- $W$ approximation as a function of $m_{t}$ for three choices of the Higgs mass: $M_{H}=100 \mathrm{GeV}, 500 \mathrm{GeV}$, and $1 \mathrm{TeV}$.

The full cross section for $e^{+} e^{-} \rightarrow t \bar{t}$ through fusion of longitudinal $W$ 's is shown in Figure 3.14 for representative values of the Higgs mass. The numerical 
results exhibit the promised enhancement for top quark masses less than $M_{H}$, as much as a factor of 10 for $M_{H}=1 \mathrm{TeV}$, and 100 for $M_{H}=500 \mathrm{GeV}$. The sum of all the $W^{+} W^{-}$contributions is graphed in Figure 3.15. For Higgs masses of 500 $\mathrm{GeV}$ and $1 \mathrm{TeV}$ the contribution from two longitudinal $W$ 's dominates.

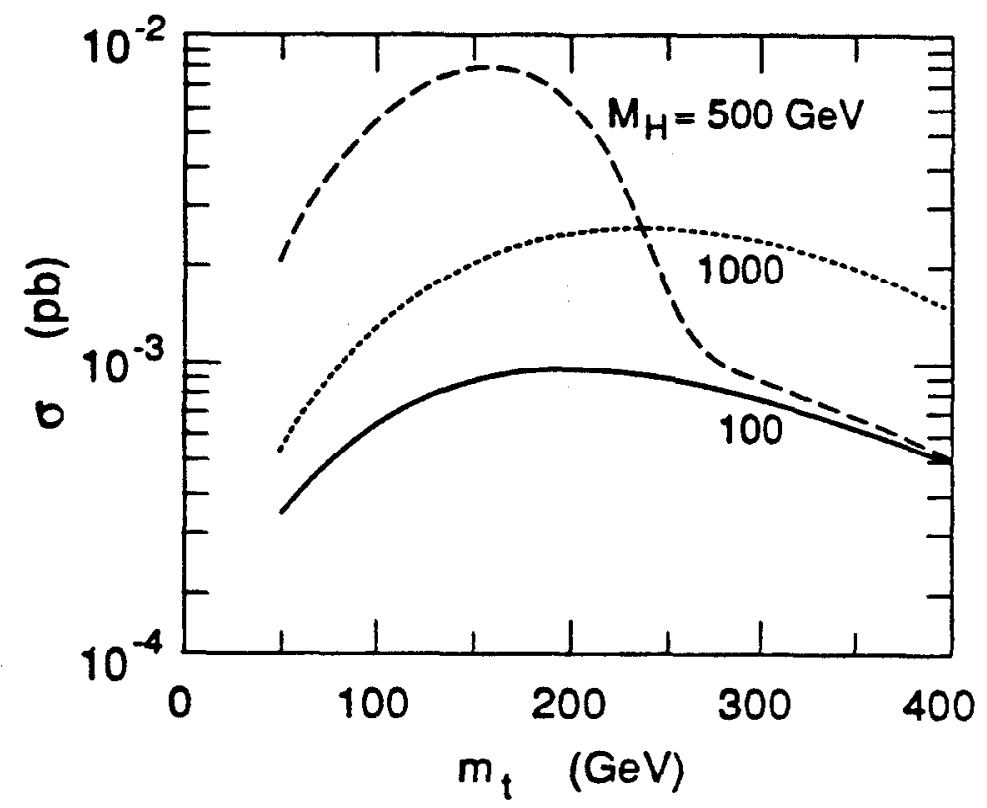

Figure 3.15 The full cross section for $e^{+} e^{-} \rightarrow \nu \bar{\nu} t \bar{t}$ at $\sqrt{s}=2 \mathrm{TeV}$ in the effective- $W$ approximation as a function of $m_{t}$ for three choices of the Higgs mass: $M_{H}=100 \mathrm{GeV}, 500 \mathrm{GeV}$, and $1 \mathrm{TeV}$.

\subsection{TOP-Quark Production from $Z Z$ Fusion}

In this section we compute the cross section for $e^{+} e^{-} \rightarrow e^{+} e^{-} t \bar{t}$ through $Z Z$ fusion, again treating the process in the effective-boson approximation. The $Z$ fusion process is easier to analyze than the $W$-fusion process because to a high degree of accuracy the distribution for the two transverse helicity states are the same. Recall that the difference between the distributions for right-handed and 
left-handed bosons is proportional to $g_{v} g_{a}$. The vector coupling of the $Z^{0}$ to the electron, $g_{v} \sim\left(-\frac{1}{4}+\sin ^{2} \theta_{w}\right)$, is negligible, since $\sin ^{2} \theta_{W} \simeq 0.23$. (This would not be the case if we were considering $Z^{0}$ 's being emitted by quarks in hadron collisions.) Thus, we are allowed to sum over the two transverse polarizations in our calculation of $Z^{0} Z^{0} \rightarrow t \bar{t}$.

The cross section for the sub-process, $Z^{0} Z^{0} \rightarrow t \bar{t}$, is readily calculated. The relevant diagrams are depicted in Figure 3.16, the two Compton-like graphs and the Higgs graph. Again, only the sum of all three graphs is well-behaved at high energies. We will present cross sections summed over the spins of the $t$ and $\bar{t}$. Just as in our $W$-fusion calculation, we will take the $Z^{0}$ momenta to be light-like, $k^{2}=0$. For polarization vectors we take those defined in Eq. (3.10) for helicity \pm 1 , and Eq. (3.14) for the longitudinal polarization.

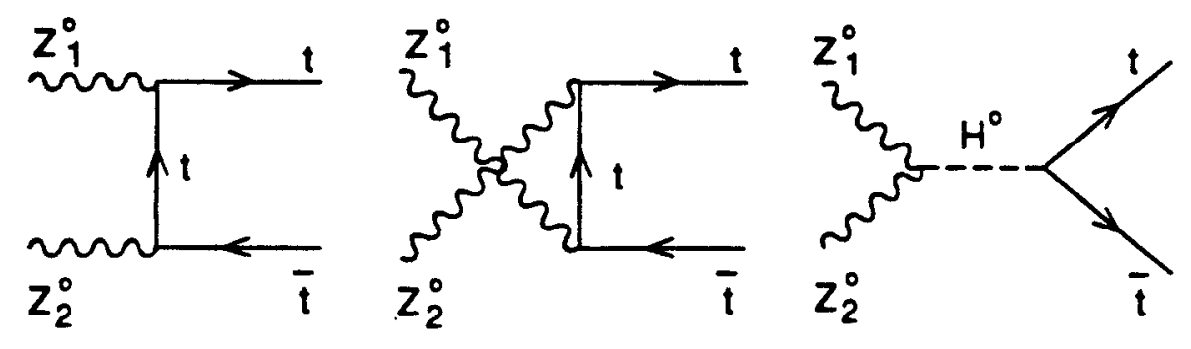

Figure 3.16 Diagrams contributing to $Z^{0} Z^{0} \rightarrow t \bar{t}$.

Let us begin with the cases where both $Z^{0}$ 's are transverse. The cross section, averaged over the two transverse polarizations is 


$$
\begin{aligned}
& \sigma\left(Z_{T}^{0} Z_{T}^{0} \rightarrow t \bar{t}\right)=\frac{\pi N_{c} \alpha^{2}}{64 s_{W}^{4} c_{W}^{4}} \frac{\beta_{t}}{\hat{s}} \times\left\{\frac { \mathcal { L } _ { t } } { \beta _ { t } } \left[1+6 c_{v}^{2}+c_{v}^{4}\right.\right. \\
& \left.-4 \Delta_{t}\left(1-2 \Delta_{t}\right)\left(3-2 c_{v}^{2}-c_{v}^{4}\right)-\Delta_{t} \operatorname{Re} X_{H}\left(1-4 \Delta_{t}\right)\left(1-c_{v}^{2}\right)\right]-1-14 c_{v}^{2}-c_{v}^{4} \\
& \left.\quad+4 \Delta_{t}\left(7+2 c_{v}^{2}-c_{v}^{4}+8 \operatorname{Re} X_{H}+4\left|X_{H}\right|^{2}\right)-64 \Delta_{t}^{2}\left(1+2 \operatorname{Re} X_{H}+\left|X_{H}\right|^{2}\right)\right\}
\end{aligned}
$$

For convenience, we have defined reduced vector and axial vector couplings of the $Z^{0}$ to the top quark:

$$
g_{v(a)}=\frac{g}{4 \cos \theta_{W}} c_{v(a)}
$$

and introduced $c_{W}=\cos \theta_{W}$. In writing Eq. (3.33) we have used $c_{a}=-1$. The other constants are defined as in the previous sections. As a check on our calculation, we can set $c_{a}=0, c_{v}=-1$, and $X_{H}=-1$ and so retrieve the form of the photonphoton result from Section 2 .

To get the full contribution from transverse $Z^{0}$ 's to $e^{+} e^{-} \rightarrow e^{+} e^{-} t \bar{t}$, we use the distributions derived in Section 4. The results for $\sqrt{s}=2 \mathrm{TeV}$ and a Higgs mass of $100 \mathrm{GeV}$ are displayed in Figure 3.17. The numerical results depend only weakly on the Higgs mass. We see that the transverse $Z^{0}$ contribution is smaller than the transverse $W$ contribution by an order of magnitude. The difference is due mainly to the smaller couplings of the $Z^{0}$, both to the electron and to the top quark. 


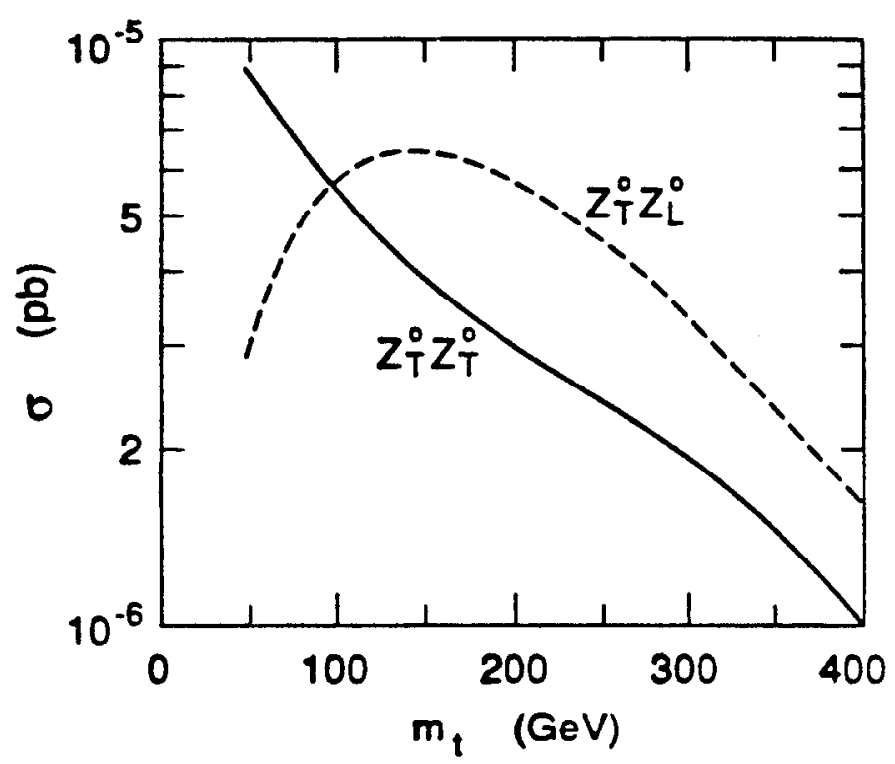

Figure 3.17 Contributions to the cross section for $e^{+} e^{-} \rightarrow e^{+} e^{-} t \bar{t}$ at $\sqrt{s}=2 \mathrm{TeV}$ from fusion of two transverse $Z^{0}$ 's and from one transverse $Z^{0}$ with a longitudinal one in the effective$Z$ approximation as a function of $m_{t}$. The Higgs mass is taken to be $M_{H}=100 \mathrm{GeV}$ but the result in very insensitive to $M_{H}$.

Now let us include the longitudinal polarization. As with the $W^{ \pm}$, we will take the longitudinal $Z^{0}$ to have a polarization vector, $\epsilon_{\mu}=k_{\mu} / M_{Z}$. Let us first present the result for one transverse and one longitudinal $Z$, averaged over the two transverse polarizations:

$$
\sigma\left(Z_{L}^{0} Z_{T}^{0} \rightarrow t \bar{t}\right)=\frac{\pi N_{c} \alpha^{2} c_{a}^{2}}{s_{w}^{4} c_{W}^{4}}\left(\frac{m_{t}}{M_{z}}\right)^{2} \frac{\beta_{t}}{\hat{s}}\left[\left(c_{v}^{2}+c_{a}^{2}\right) \frac{\mathcal{L}_{t}}{\beta_{t}}-2 c_{a}^{2}\right] .
$$

As we found for $W_{\lambda= \pm 1}^{+} W_{\lambda=0}^{-} \rightarrow t \bar{t}$, Eq. (3.35) is proportional to $\left(m_{t} / M_{z}\right)^{2}$ and independent of the Higgs mass. Note also that the result is proportional to $c_{a}^{2}$, since without the axial coupling the $Z^{0}$ would mimic the photon and its longitudinal mode would not couple. (An on-shell $Z^{0}$ would also have a vector amplitude, but it would be suppressed by $M_{z} / \hat{s}$.) The result after folding in the effective- $Z$ distributions is shown in Figure 3.17; it is again much smaller than the equivalent $W$-fusion process. 


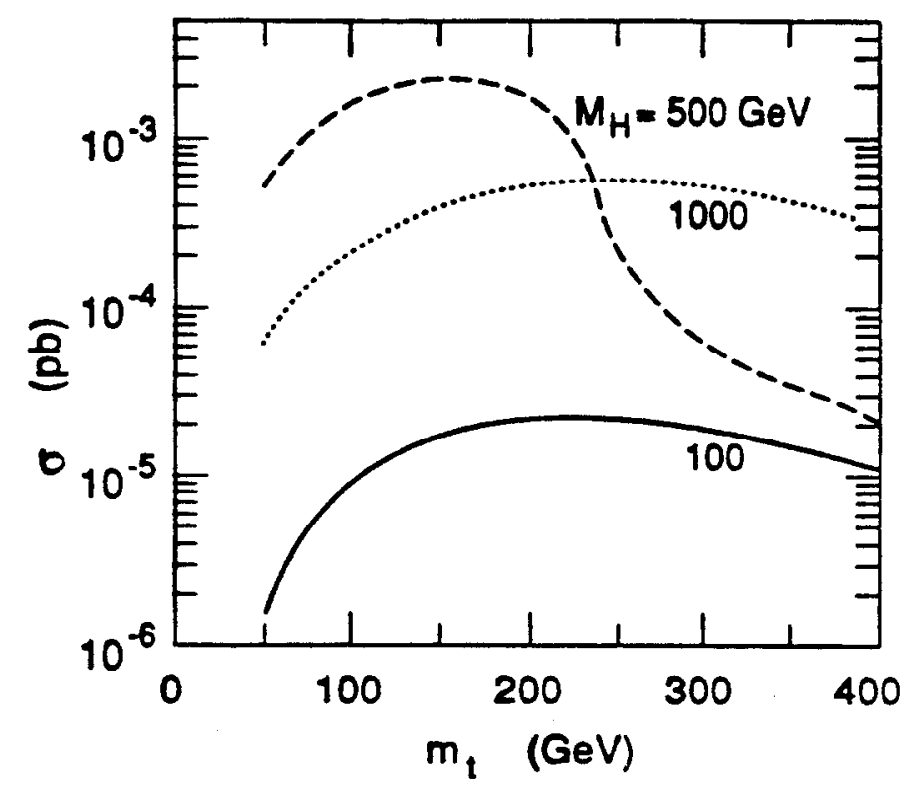

Figure 3.18 Contributions to the cross section for $e^{+} e^{-} \rightarrow e^{+} e^{-} t \bar{t}$ at $\sqrt{s}=2 \mathrm{TeV}$ from fusion of two longitudinal $Z^{0}$ 's in the effective $Z$ approximation as a function of $m_{t}$ for three choices of the Higgs mass: $M_{H}=100 \mathrm{GeV}, 500 \mathrm{GeV}$, and $1 \mathrm{TeV}$.

Finally, consider the process with both $Z$ 's longitudinally polarized. The cross section is

$$
\sigma\left(Z_{L}^{0} Z_{L}^{0} \rightarrow t \bar{t}\right)=\frac{\pi N_{c} \alpha^{2}}{4 s_{W}^{4} c_{W}^{4}}\left(\frac{m_{t}}{M_{z}}\right)^{4} \frac{\beta_{t}}{\hat{s}}\left[\left(1+2 \operatorname{Re} X_{H}\right)\left(\frac{\mathcal{L}_{t}}{\beta_{t}}-2\right)+\left|X_{H}\right|^{2}\left(\frac{1}{2 \Delta_{t}}-2\right)\right]
$$

It has the expected factor of $\left(m_{t} / M_{z}\right)^{4}$. The result also contains a factor of $c_{a}^{4}=1$, since each $Z_{L}^{0}$ couples with a power of $c_{a}$. Repeating the familiar procedure of integrating over the effective fluxes we obtain the contribution of longitudinal $Z^{0}$ 's to $e^{+} e^{-} \rightarrow e^{+} e^{-} t \bar{t}$. The full results are shown in Figure 3.18 for our three canonical values of the Higgs mass. We see again the delayed unitarity cancellation for the larger two choices of the Higgs mass. The sum of all $Z Z$ fusion contributions to top-quark production is shown in Figure 3.19 for the same three choices of the Higgs mass. The contribution from longitudinal Z's dominates for the whole 


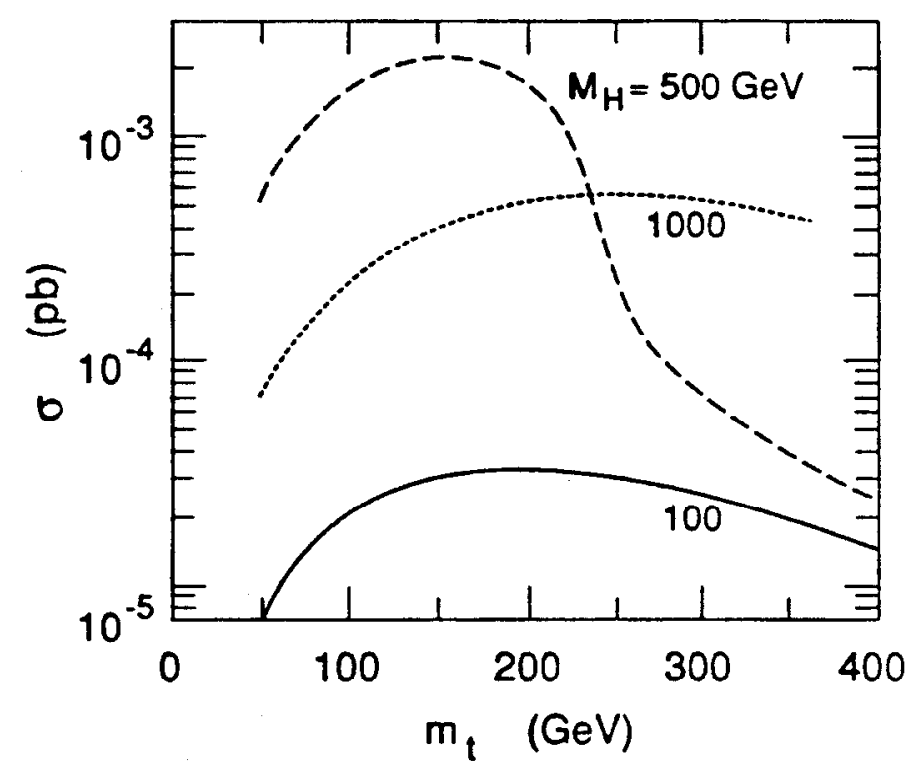

Figure 3.19 The sum of the contributions to the cross section for $e^{+} e^{-} \rightarrow e^{+} e^{-} t \bar{t}$ at $\sqrt{s}=$ $2 \mathrm{TeV}$ from fusion of $Z^{0}$ 's in the effective- $Z$ approximation as a function of $m_{t}$ for three choices of the Higgs mass: $M_{H}=100 \mathrm{GeV}, 500 \mathrm{GeV}$, and $1 \mathrm{TeV}$.

considered range of $m_{t}$ if $M_{H}=500$ or $1000 \mathrm{GeV}$, and for $m_{t}>200 \mathrm{GeV}$ for $M_{H}=100 \mathrm{GeV}$.

\subsection{Photon $-Z^{0}$ Fusion Production of TOP QUarks}

Leaving no stone unturned, we now direct out attention to the production of $t \bar{t}$ pairs through fusion of a photon and a $Z^{0}$. The diagrams are the same as those for photon-photon fusion, with one of the photons replaced by a $Z^{0}$. They are depicted in Figure 3.20.

The calculation of the cross section for the sub-process $\gamma Z^{0} \rightarrow t \bar{t}$ holds no subtleties; we simply add the two amplitudes, square the result and integrate over the $t \bar{t}$ phase space. Since the distribution of transverse $Z^{0}$ 's in the electron is very nearly independent of the helicity, we will average over the transverse helicities. 

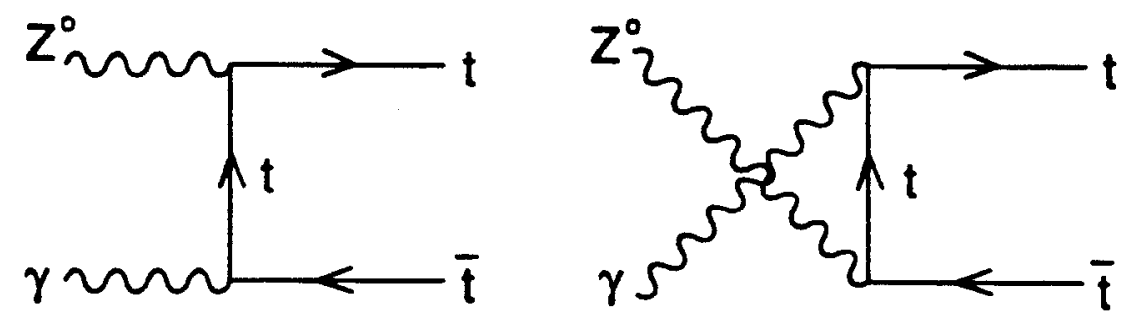

Figure 3.20 Diagrams contributing to $\gamma Z^{0} \rightarrow t \bar{t}$.

The cross section for transverse $Z^{0}$ 's, averaged over polarizations, is

$$
\sigma\left(\gamma Z_{T} \rightarrow t \bar{t}\right)=\frac{\pi N_{c} Q_{t}^{2} \alpha^{2}}{2 s_{W}^{2} c_{w}^{2}} \frac{\beta_{t}}{\hat{s}}\left\{\frac{\mathcal{L}_{t}}{\beta_{t}}\left(c_{l}^{2}+c_{r}^{2}+8 c_{l} c_{r} \Delta_{t}-16 c_{l} c_{r} \Delta_{t}^{2}\right)-c_{l}^{2}-c_{r}^{2}-8 c_{l} c_{r}\right\}
$$

where $c_{l}$ and $c_{r}$ are reduced right-handed and left-handed couplings of the $Z^{0}$ to the top quark:

$$
\begin{aligned}
& c_{l}=1-2 Q_{t} s_{W}^{2} \\
& c_{r}=-2 Q_{t} s_{W}^{2} .
\end{aligned}
$$

The cross section for photon-photon fusion to top quarks is regained if we set $c_{l}=c_{r}=Q_{t}$.

The cross section for the case of longitudinal $Z^{0}$ 's is equally easy to evaluate. The result, averaged over photon spins, is

$$
\sigma\left(\gamma Z_{L} \rightarrow t \bar{t}\right)=\frac{\pi N_{c} Q_{t}^{2} \alpha^{2}}{s_{w}^{2} c_{W}^{2}}\left(\frac{m_{t}}{M_{z}}\right)^{2}\left(c_{l}-c_{r}\right)^{2} \frac{\mathcal{L}_{t}}{\hat{s}}
$$

Our result contains the expected factor of $\left(m_{t} / M_{z}\right)^{2}$. Since the longitudinal $Z^{0}$ couples axially at high energies, our result is also proportional to $\left(c_{l}-c_{r}\right)^{2}=1$. 


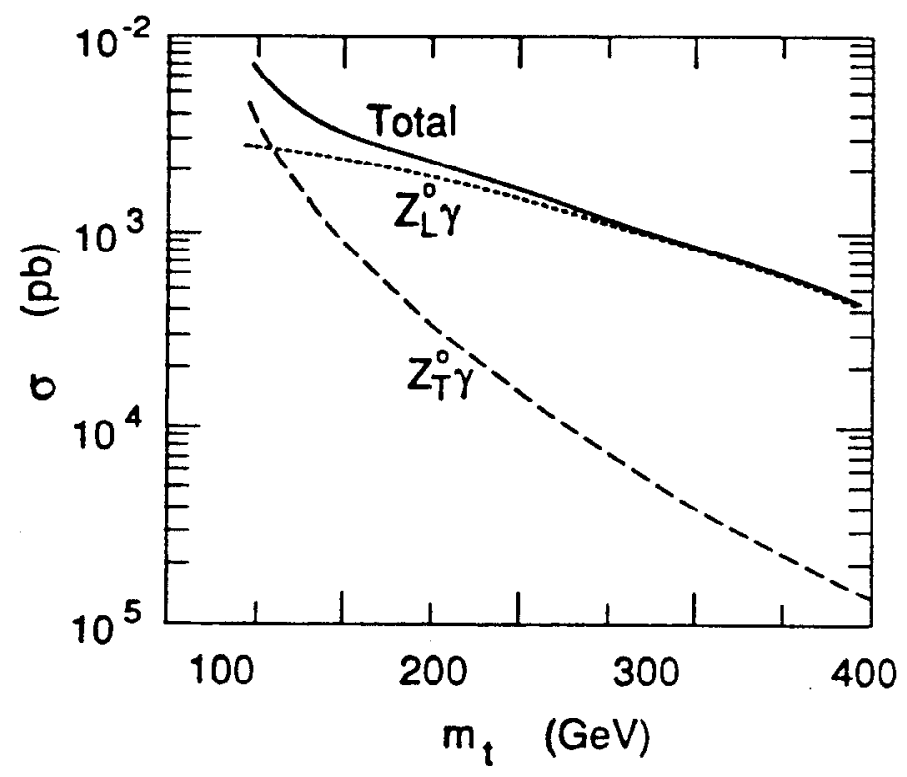

Figure 3.21 Contributions to the cross section for $e^{+} e^{-} \rightarrow e^{+} e^{-} t \bar{t}$ at $\sqrt{s}=2 \mathrm{TeV}$ from fusion of a photon with a $Z^{0}$ in the effective-boson approximation for both photon and $Z^{0}$ as a function of $m_{t}$.

The convolution of these cross sections with the relevant distributions yields the portion of the $e^{+} e^{-} \rightarrow e^{+} e^{-} t \bar{t}$ cross section due to photon- $Z^{0}$ fusion. The results of the numerical integration are displayed in Figure 3.21. We see that the transverse contribution is very small, two orders of magnitude below the photonphoton result. The enhancement of the longitudinal mode allows the longitudinal result to surpass the two-photon result for top masses above $300 \mathrm{GeV}{ }^{29}$

\subsection{PHOTON-Z $Z^{0}$ INTERFERENCE}

In the previous sections we have discussed the effective-boson approximation for processes involving the photon, $Z^{0}$ and $W^{ \pm}$. Our discussion has neglected the fact that amplitudes involving the photon may interfere with amplitudes involving the $Z^{0}$. There is no a priori reason why these amplitudes should vanish. In this section we derive the analogue of the effective-boson approximation for these 

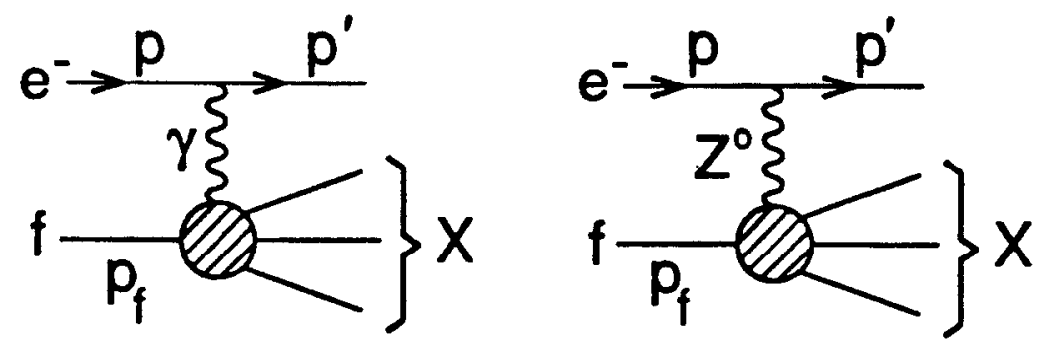

Figure 3.22 Production of an arbitrary final state $X$ in a collision of an electron with an arbitrary initial particle $f$ by exchange of a photon or a $Z^{0}: e^{-} f \rightarrow e^{-} X$.

interference terms. We then proceed to calculate the interference contribution to $e^{+} e^{-} \rightarrow e^{+} e^{-} t \bar{t}$ using our formalism.

The derivation proceeds in complete analogy to our previous derivations of the effective-boson approximation for photons and heavy vector bosons. We begin by considering the process depicted in Figure 3.22, $e^{-} f \rightarrow e^{-} X$, for some particle $f$ and final state $X$, both arbitrary. The full amplitude is a sum of the photon and $Z^{0}$ diagrams:

$$
\mathcal{M}=\bar{u}\left(p^{\prime}\right)\left[\frac{e \mathcal{A}_{e}^{\mu}}{k^{2}}-\frac{\left(g_{v}+g_{a} \gamma^{5}\right) \mathcal{A}_{z}^{\mu}}{k^{2}-M_{z}^{2}}\right] \gamma_{\mu} u(p)
$$

where, as before, $\mathcal{A}_{e}$ is the three-point coupling $\gamma f \rightarrow X$, and $\mathcal{A}_{z}$ is the analogous quantity for the $Z^{0}$. When we square the amplitude and average over the spin of the electron we find

$$
|\overline{\mathcal{M}}|^{2} \equiv \frac{1}{2} \sum_{\text {spins }}|\mathcal{M}|^{2}=\left|\overline{\mathcal{M}}_{z z}\right|^{2}+\left|\overline{\mathcal{M}}_{\gamma \gamma}\right|^{2}+\left|\overline{\mathcal{M}}_{\text {int }}\right|^{2}
$$


where the interference contribution is

$$
\begin{aligned}
\left|\overline{\mathcal{M}}_{\mathrm{int}}\right|^{2}=-\frac{2 e}{k^{2}\left(k^{2}-M_{z}^{2}\right)}\{ & \left.g_{v}\left[p^{\mu} p^{\prime \alpha}+p^{\alpha} p^{\prime \mu}-\left(p \cdot p^{\prime}\right) g^{\mu \alpha}\right]+i g_{a} \epsilon^{\mu \alpha \rho \sigma} p_{\rho} p_{\sigma}^{\prime}\right\} \\
& \times g_{\mu \nu} g_{\alpha \beta}\left(\mathcal{A}_{e}^{\nu} \mathcal{A}_{z}^{* \beta}+\mathcal{A}_{z}^{\nu} \mathcal{A}_{e}^{* \beta}\right) .
\end{aligned}
$$

When we replace the propagators by polarization sums [Eq. (3.9)] we obtain

$$
\begin{aligned}
\left|\overline{\mathcal{M}}_{\mathrm{int}}\right|^{2}=- & \frac{2 e}{k^{2}\left(k^{2}-M_{z}^{2}\right)} \sum_{i j}\left\{g_{v}\left[\left(p \cdot \epsilon_{i}^{*}\right)\left(p^{\prime} \cdot \epsilon_{j}\right)+\left(p \cdot \epsilon_{j}\right)\left(p^{\prime} \cdot \epsilon_{i}^{*}\right)-\left(p \cdot p^{\prime}\right)\left(\epsilon_{i}^{*} \cdot \epsilon_{j}\right)\right]\right. \\
& \left.+i g_{a} \epsilon^{\mu \alpha \rho \sigma} \epsilon_{i \mu}^{*} \epsilon_{j \alpha} p_{\rho} p_{\sigma}^{\prime}\right\}\left[\left(\mathcal{A}_{e} \cdot \epsilon_{i}\right)\left(\mathcal{A}_{z}^{*} \cdot \epsilon_{j}^{*}\right)+\left(\mathcal{A}_{z} \cdot \epsilon_{i}\right)\left(\mathcal{A}_{e}^{*} \cdot \epsilon_{j}^{*}\right)\right],
\end{aligned}
$$

where the sum runs over the three polarizations of the intermediate photon or $Z^{0}$ : $i, j=0, \pm 1$. We see immediately that Eq. (3.43) has the same structure that we observed in the effective-boson approximation, Eq. (3.11). The same argument we used in that case shows to that the terms in the double sum for which $i \neq j$ vanish, either identically or after integration over the azimuthal angle of the electron.

As we did in the effective-photon approximation we will neglect the longitudinal coupling of the photon. Thus the sum in Eq. (3.43) runs only over transverse polarizations. When we insert the explicit polarization vectors defined in Eq. (3.10) into $\mathrm{Eq}$ (3.43), dropping the off-diagonal and longitudinal terms in the sum, we find

$$
\begin{aligned}
\left|\overline{\mathcal{M}}_{\text {int }}\right|^{2}= & -\frac{2 e}{k^{2}\left(k^{2}-M_{z}^{2}\right)} \sum_{\lambda= \pm 1}\left\{g_{v}\left(p_{\perp}^{2}-\frac{1}{2} k^{2}\right)-\lambda g_{a}\left(E p_{3}^{\prime}-E^{\prime} p_{3}\right)\right\} \\
& \times\left[\left(\mathcal{A}_{e} \cdot \epsilon_{\lambda}\right)\left(\mathcal{A}_{z}^{*} \cdot \epsilon_{\lambda}^{*}\right)+\left(\mathcal{A}_{z} \cdot \epsilon_{\lambda}\right)\left(\mathcal{A}_{e}^{*} \cdot \epsilon_{\lambda}^{*}\right)\right] .
\end{aligned}
$$

Note that the term proportional to $g_{a}$ in Eq. (3.44) is proportional to the helicity $\lambda$. When we repeat our argument for the case in which the photon and $Z^{0}$ are emitted 
from a positron we find that this term also changes sign, the sign difference coming from an interchange of $p$ with $p^{\prime}$. We expect the axial portion of the distribution to dominate since the vector coupling of the $Z^{0}$ to the electron is small; however, the sign flip from electron to positron will cause some of these terms to cancel, as we will see.

We now invoke the assumptions of the effective-boson approximation: we replace the amplitudes $\mathcal{A}_{e}$ and $\mathcal{A}_{z}$ with their values at $k^{2}=0$ so that they can be removed from the angular integral. We define an interference cross section

$$
\sigma_{\lambda}^{\mathrm{int}}\left(\left\{\gamma \mid Z^{0}\right\} f \rightarrow X\right)=\int d \Gamma\left[\left(\mathcal{A}_{e} \cdot \epsilon_{\lambda}\right)\left(\mathcal{A}_{z}^{*} \cdot \epsilon_{\lambda}^{*}\right)+\left(\mathcal{A}_{z} \cdot \epsilon_{\lambda}\right)\left(\mathcal{A}_{e}^{*} \cdot \epsilon_{\lambda}^{*}\right)\right]
$$

where $d \Gamma$ is the invariant phase space of the state $X$. We write the contribution of the interference terms to the full cross section as

$$
\sigma^{\mathrm{int}}\left(e^{-} f \rightarrow e^{-} X\right)=\left.\int d x \sum_{\lambda} f_{\lambda}^{\mathrm{int}}(x) \sigma^{\mathrm{int}}\left(\left\{\gamma(\lambda) \mid Z^{0}(\lambda)\right\} f \rightarrow X\right)\right|_{\hat{s}=x s}
$$

Just as we did in Section 4, we can read off the interference distributions:

$$
f_{\lambda}^{\text {int }}(x)=-\frac{e}{(2 \pi)^{2}} E^{\prime} \omega \int \frac{d \cos \theta}{k^{2}\left(k^{2}-M_{z}^{2}\right)}\left\{g_{v}\left(p_{\perp}^{2}-\frac{1}{2} k^{2}\right)-\lambda g_{a}\left(E p_{3}^{\prime}-E^{\prime} p_{3}\right)\right\}
$$

For convenience, we break up the distributions into vector and axial vector pieces:

$$
\begin{aligned}
& f_{v}^{\text {int }}(x)=-\frac{e g_{v}}{(2 \pi)^{2}} E^{\prime} \omega \int \frac{d \cos \theta}{k^{2}\left(k^{2}-M_{z}^{2}\right)}\left(p_{\perp}^{2}-\frac{1}{2} k^{2}\right), \\
& f_{a}^{\text {int }}(x)=\frac{e g_{a}}{(2 \pi)^{2}} E^{\prime} \omega \int \frac{d \cos \theta}{k^{2}\left(k^{2}-M_{z}^{2}\right)}\left(E p_{3}^{\prime}-E^{\prime} p_{3}\right) .
\end{aligned}
$$




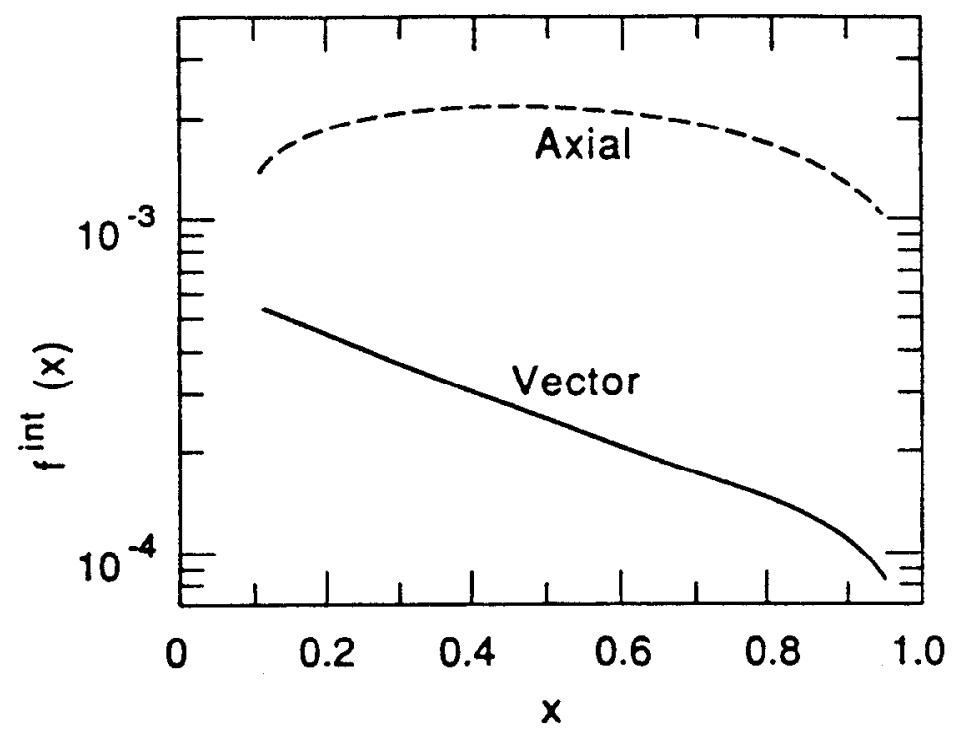

Figure 3.23 The interference distributions [Eqs.(3.49) and (3.50)] for a photon interfering with a $Z^{0}$ emitted from an electron at energy $1 \mathrm{TeV}$ as a function of the momentum fraction $x$.

When we insert the kinematics from Eq. (3.7) and perform the integrals, we find

$$
\begin{aligned}
& f_{v}^{\text {int }}(x)=-\frac{e g_{v}}{8 \pi^{2} x \eta^{2}}\left\{\log \left[\frac{1-x+\Delta_{z}}{\Delta_{z}}\right]\left(1-\Delta_{z}-x+\frac{x^{2}}{2}\right)-\log \left[\frac{2-x}{x}\right] \frac{(2-x)^{2}}{2}\right\} \\
& f_{a}^{\text {int }}(x)=\frac{e g_{a}}{16 \pi^{2} \eta}\left\{\log \left[\frac{2-x-x \eta}{2-x+x \eta}\right]+\log \left[\frac{1+\eta}{1-\eta}\right]\right\}
\end{aligned}
$$

where $\eta$ and $\Delta_{z}$ are defined as before, $\Delta_{z}=M_{z}^{2} / s$ and $\eta=\sqrt{1-\Delta_{z} / x^{2}}$. The interference distributions are plotted in Figure 3.23. The axial distribution is between a factor of 2 and 10 times as large as the vector distribution.

Now that we have developed our formalism we can proceed to calculate the contribution to $e^{+} e^{-} \rightarrow e^{+} e^{-} t \bar{t}$ from the interference of diagrams involving different numbers of photons and $Z^{0}$ 's. The complete set of diagrams are shown in Figure 3.24. Brute force procedures could be used to calculate each amplitude and interfere it with each of the others, followed by integration of the terms over the 

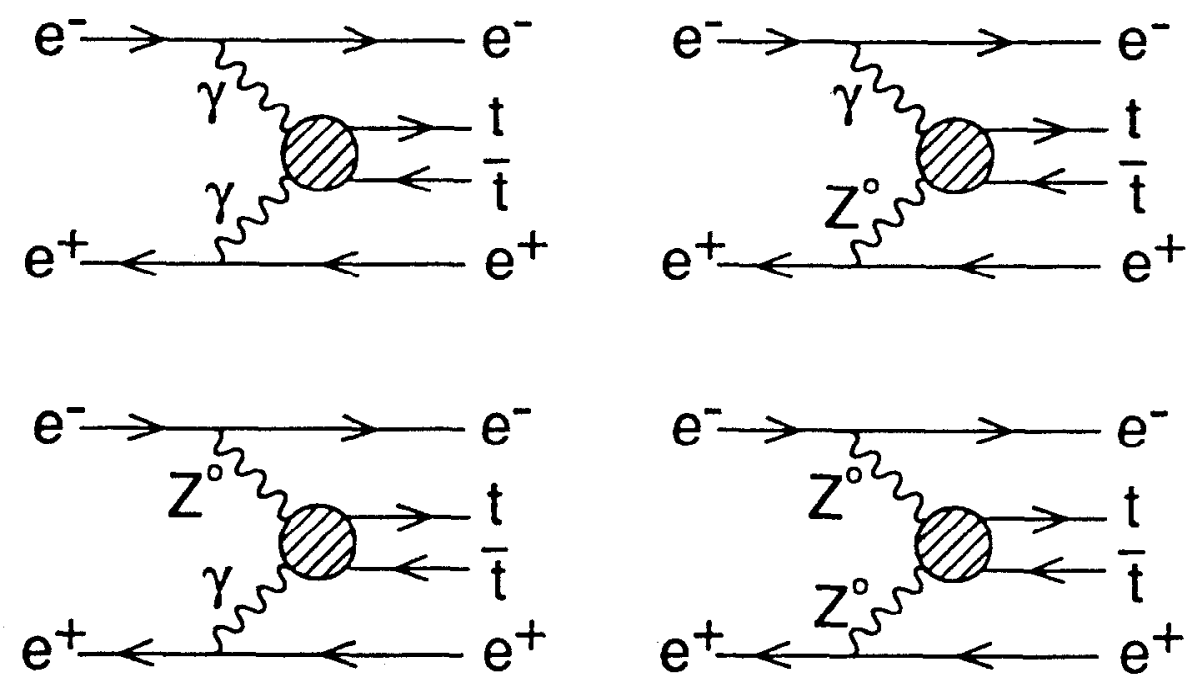

Figure 3.24 The set of diagrams contributing to the cross section for $e^{+} e^{-} \rightarrow e^{+} e^{-} t \bar{t}$ in the effective-boson approximation.

appropriate distributions. However, since therc are many contributing amplitudes, we will use our knowledge of the distributions to pick out the dominant terms and compute those only.

Since the photon distributions are much larger than the $Z^{0}$ distributions we might naively expect the dominant contributions to come from interference terms involving the maximum number of photons. The terms with the maximum number of photons are products of a photon-photon fusion diagram with a photon- $Z^{0}$ fusion diagram as shown in Figure 3.25a. However, the axial portion of this contribution will be cancelled by the mirror-image term, shown in Figure $3.25 \mathrm{~b}$, the product of a photon-photon diagram with a photon- $Z^{0}$ diagram with the photon being emitted by the positron and not the electron. The piece left over contains only the "vector" distribution, proportional to $g_{v}$, which is considerably smaller than the axial distribution. Similarly the terms involving three $Z^{0}$ 's and one pho- 


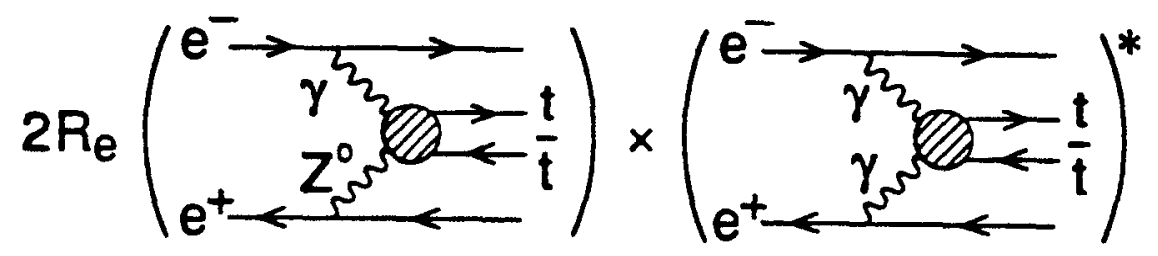

(a)
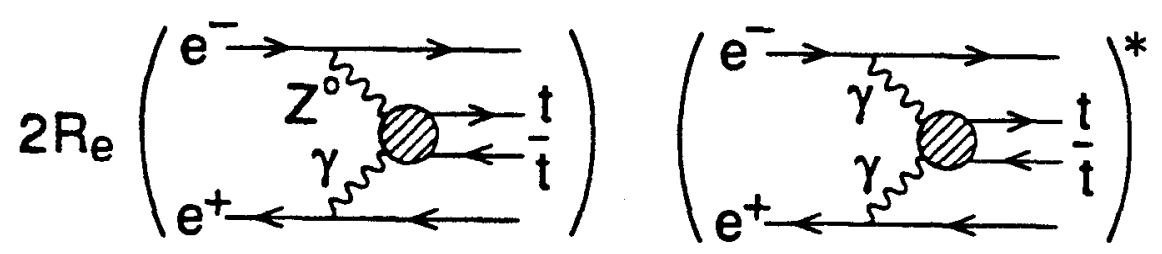

(b)

Figure 3.25 The diagrams representing terms involving three photons and one $Z^{0}$. The axial portion from the term in a), where the $Z^{0}$ is on the positron line, cancels with the axial portion of the term in $\mathrm{b}$ ), where the $Z^{0}$ is on the electron line.

ton will only receive vector contributions; we will neglect these terms completely, since the flux of $Z^{0}$ 's in the electron is much smaller than the flux of photons. Contributions from the "axial" distribution will cancel out from all terms except those shown in Figure 3.26. These are the diagrams which are symmetric with respect to interchange of the electron and positron. So we expect the leading terms to be those involving three photons with a single $Z^{0}$ and those involving two photons and two $Z^{0}$ 's.

We begin with the interference terms containing three photons and one $Z^{0}$. It is straightforward to calculate the product of the two amplitudes and integrate over the appropriate phase space. Since the "vector" interference distribution is independent of helicity we can sum over the helicities of the interfering bosons; we average over the helicity of the photon. An additional factor of two comes from the fact that the $Z^{0}$ can be emitted from the electron or the positron. The result 

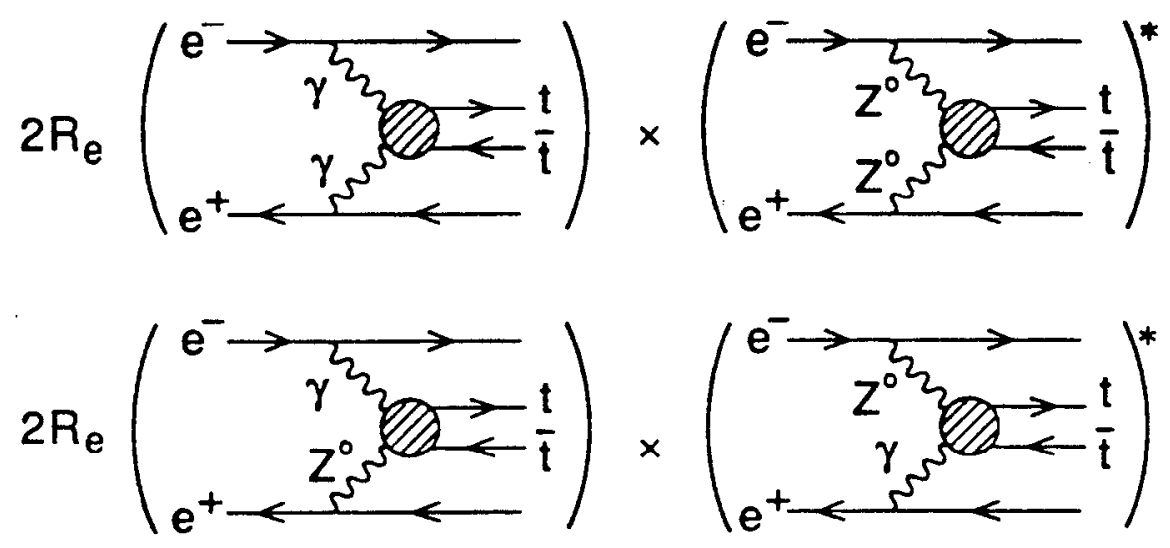

Figure 3.26 The diagrams representing the terms that are symmetric with respect to the interchange of the electron and positron. These are the only terms that receive a contribution from the axial distribution.

is:

$$
\begin{aligned}
\bar{\sigma}^{\text {int }} & =2 \cdot \frac{1}{2} \sum_{\lambda_{1} \lambda_{2}} \sigma^{\text {int }}\left(\left\{\gamma\left(\lambda_{1}\right) \mid Z^{0}\left(\lambda_{1}\right)\right\} \gamma\left(\lambda_{2}\right) \rightarrow t \bar{t}\right) \\
& =\frac{4 \pi Q_{t}^{3} \alpha^{2}}{s_{W} c_{W}} \frac{\beta}{\hat{s}}\left(c_{l}+c_{r}\right)\left\{\frac{\mathcal{L}}{\beta_{t}}\left(1+4 \Delta_{t}-8 \Delta_{t}^{2}\right)-1-4 \Delta_{t}\right\},
\end{aligned}
$$

where $c_{l}$ and $c_{r}$ describe the right- and left-handed couplings of the $Z^{0}$ to the top quark defined in Eq. (3.38). The polarization sum runs over $\lambda_{1}, \lambda_{2}= \pm 1$. The astute reader will recognize that Eq. (3.51) has the same form as the cross section for $\gamma \gamma \rightarrow t \bar{t}$. Note also that this "cross section" is positive.

The interference terms involving two photons and two $Z^{0}$ 's are also straightforward to calculate. In this case we do not average over polarizations. Since the sign of the "axial" distribution alternates with the helicity we calculate the sum over polarizations weighted by a factor of $\lambda_{1} \lambda_{2}$ :

$$
\tilde{\sigma}^{\text {int }}=\sum_{\lambda_{1} \lambda_{2}} \lambda_{1} \lambda_{2} \sigma^{\text {int }}\left(\left\{\gamma\left(\lambda_{1}\right) \mid Z^{0}\left(\lambda_{1}\right)\right\}\left\{\gamma\left(\lambda_{2}\right) \mid Z^{0}\left(\lambda_{2}\right)\right\} \rightarrow t \bar{t}\right)
$$


We calculate the terms depicted in Figure 3.26 integrated over the $t-\bar{t}$ phase space:

$$
\tilde{\sigma}^{\text {int }}=-\frac{8 \pi \alpha^{2}}{s_{W}^{2} c_{W}^{2}} \frac{\beta_{t}}{\hat{s}}\left\{\frac{\mathcal{L}_{t}}{\beta_{t}}\left[c_{l}^{2}+c_{r}^{2}-\Delta_{t}\left(c_{l}-c_{r}\right)^{2}\right]-c_{l}^{2}-c_{r}^{2}-c_{l} c_{r}\right\} .
$$

Note that this "cross section" is negative.

The contribution to $e^{+} e^{-} \rightarrow e^{+} e^{-} t \bar{t}$ are then obtained by folding in the appropriate distributions:

$$
\begin{aligned}
& \sigma_{v}^{\mathrm{int}}\left(e^{+} e^{-} \rightarrow e^{+} e^{-} t \bar{t}\right)=\left.\int d x_{1} d x_{2} f_{v}^{\mathrm{int}}\left(x_{1}\right) f_{\gamma}\left(x_{2}\right) \bar{\sigma}^{\mathrm{int}}\right|_{\hat{s}=x_{1} x_{2} s} \\
& \sigma_{a}^{\mathrm{int}}\left(e^{+} e^{-} \rightarrow e^{+} e^{-} t \bar{t}\right)=-\left.\int d x_{1} d x_{2} f_{a}^{\mathrm{int}}\left(x_{1}\right) f_{a}^{\mathrm{int}}\left(x_{2}\right) \tilde{\sigma}^{\mathrm{int}}\right|_{\hat{s}=x_{1} x_{2} s} .
\end{aligned}
$$

The factor of -1 in Eq. (3.54) comes from the axial interference distribution off of the positron. Since $\widetilde{\sigma}^{\text {int }}$ is negative, both contributions are positive. The numerical integration of Eqs. (3.53) and (3.54) are plotted in Figure 3.27. The two contributions are of roughly equal magnitude, the vector contribution dominates at smaller masses since the vector distribution is peaked at small $\mathrm{x}$ while the axial distribution is relatively flat. We might naively expect the interference to be larger than the $\gamma Z^{0}$-fusion contribution. This is not the case due to the cancellation of the leading axial terms. Instead, the interference contribution is roughly comparable with that from fusion of transverse $Z^{0}$ s, two orders of magnitude less than the $\gamma Z^{0}$ contribution. 


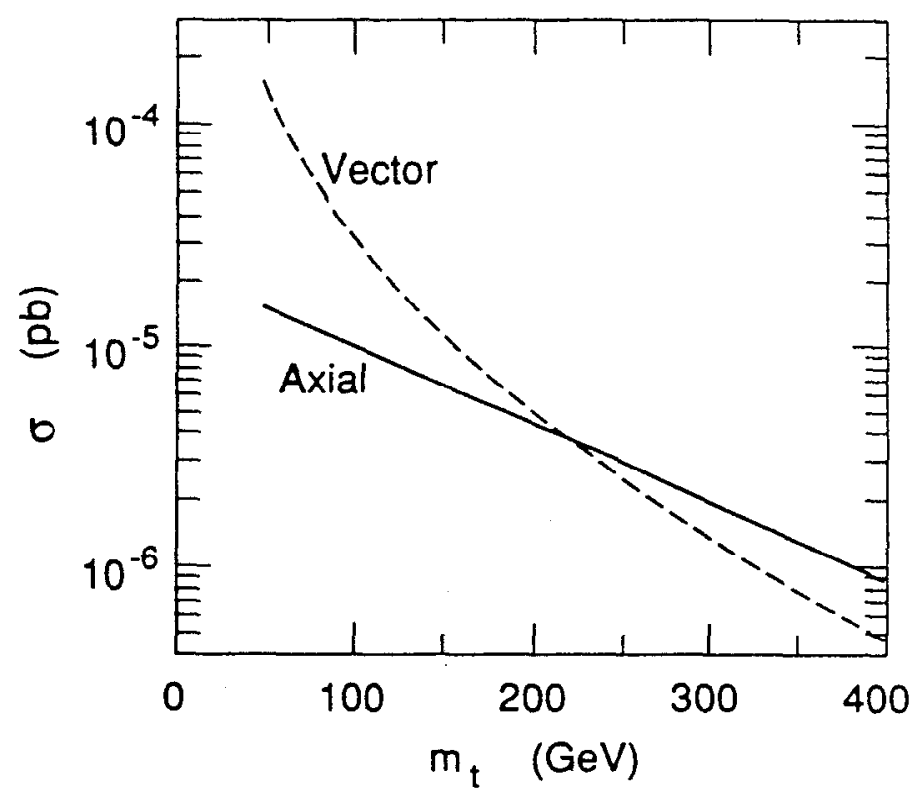

Figure 3.27 The contributions to the cross section for $e^{+} e^{-} \rightarrow e^{+} e^{-} t \bar{t}$ at $\sqrt{s}=2^{\mathrm{T}} \mathrm{TeV}$ from interference among diagrams involving photons and $Z^{0}$ 's as a function of $m_{t}$.

\subsection{Production of $t \bar{b}$ Pairs from $\gamma W$ Fusion}

There exists another vector boson fusion process which is capable of producing top quarks. In this section we will calculate the production of $t \vec{b}$ pairs through the fusion of a photon and $W^{+}$. This process has an advantage over the $t \bar{t}$ processes: it has a lower threshold energy, since the bottom quark is much lighter than the top quark. Since the cross section for the subprocess of two bosons going to fermion and anti-fermion goes like $1 / \hat{s}$, the lower threshold provides an effective enhancement. Furthermore, the effective-photon flux grows at the lower momentum fractions allowed in this process. Finally, the $\gamma W$ fusion process involves a bottom quark propagator which may become nearly on shell in the forward direction, causing an enhancement proportional to $\log \left(\hat{s} / m_{b}^{2}\right)$.

We will first treat the process in the effective- $W$ approximation, even though 


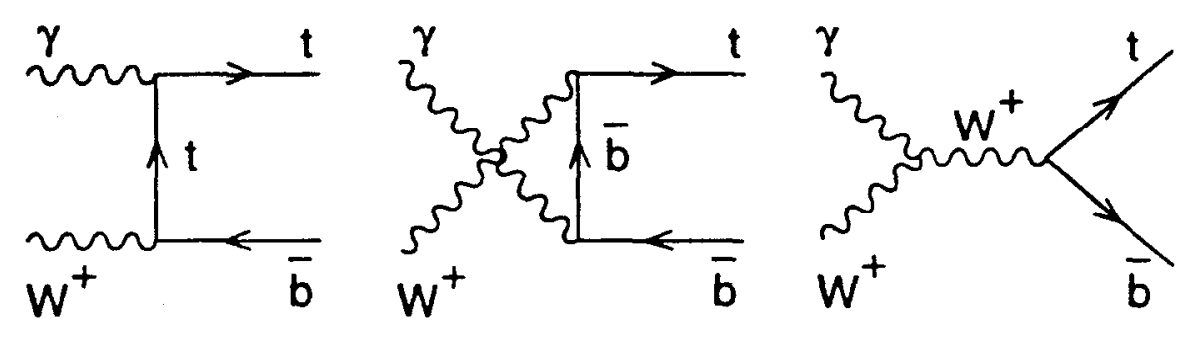

Figure 3.28 Feynman diagrams for the process $\gamma W^{+} \rightarrow \imath \bar{b}$.

this is an occasion in which we do not have much trust in the accuracy of the approximation. The effective- $W$ approximation breaks down when the energy of the virtual $W$ does not exceed its mass. There are parts of the phase space for which this is the case in the production of $t \bar{b}$ pairs. However, these parts of the phase space do not contribute the bulk of the cross section. Rather it is configurations where the photon is at low $x$ and the $W$ at relatively high $x$ which will dominate. These parts of the phase space are well described by the effective- $W$ approximation.

Fusion of a $W^{+}$and a photon to form $t \bar{b}$ proceeds through the diagrams in Figure 3.28. The photon may couple to the $t$ quark or the anti-b. (Note that this second diagram is absent in the analogous leptonic process: $\gamma W \rightarrow l \nu$.) We will restrict ourselves to top quark masses above $M_{W}$, so that the $s$-channel $W$ will never be on-shell. We will present the analytic forms of the cross sections for the sub-process $\gamma W^{+} \rightarrow t \bar{b}$, with the different helicity combinations treated separately. Our numerical results will averaged over the polarization of the photon, since the effective-photon distributions are polarization independent.

The calculations of the cross sections are easily carried out. The results for 
transverse $W$ 's are:

$$
\begin{gathered}
\sigma\left(\gamma_{\lambda=+1} W_{\lambda=+1}^{+} \rightarrow t \bar{b}\right)=\frac{\pi N_{c} \alpha^{2}}{18 s_{W}^{2} \hat{s}}\left\{-24 \Delta_{t} \mathcal{L}_{t}^{\prime}\left[1-\Delta_{t}+X_{W}\left(1+\Delta_{t}\right)\right]\right. \\
\left.+\left(1-\Delta_{t}\right)\left[16+6 X_{W}^{2}-\Delta_{t}\left(7-48 X_{W}+3 X_{W}^{2}\right)-3 \Delta_{t}^{2}\left(3+X_{W}^{2}\right)\right]\right\} \\
\sigma\left(\gamma_{\lambda=+1} W_{\lambda=-1}^{+} \rightarrow t \bar{b}\right)=\frac{\pi N_{c} \alpha^{2}}{9 s_{W}^{2} \hat{s}}\left\{2 \mathcal{L}_{b}^{\prime}\left(1-\Delta_{t}\right)^{2}+8 \Delta_{t}^{2} \mathcal{L}_{t}^{\prime}-\left(1-\Delta_{t}\right)\left(7-5 \Delta_{t}+6 \Delta_{t}^{2}\right)\right\} \\
\sigma\left(\gamma_{\lambda=-1} W_{\lambda=+1}^{+} \rightarrow t \bar{b}\right)=\frac{\pi N_{c} \alpha^{2}}{9 s_{W}^{2} \hat{s}}\left\{8 \mathcal{L}_{t}^{\prime}\left(1+5 \Delta_{t}+3 \Delta_{t}^{2}\right)-\left(1-\Delta_{t}\right)\left(25+41 \Delta_{t}+6 \Delta_{t}^{2}\right)\right\} \\
\sigma\left(\gamma_{\lambda=-1} W_{\lambda=-1}^{+} \rightarrow t \bar{b}\right)=\frac{\pi N_{c} \alpha^{2}}{18 s_{w}^{2} \hat{s}}\left\{-8 \Delta_{t}^{2} \mathcal{L}_{t}^{\prime}\left(1+3 X_{W}\right)+4 \Delta_{t}^{2} \mathcal{L}_{b}^{\prime}\right. \\
\left.+\left(1-\Delta_{t}\right)\left[4+6 X_{W}^{2}+\Delta_{t}\left(13+12 X_{W}-3 X_{W}^{2}\right)\right]\right\}
\end{gathered}
$$

In writing Eqs. (3.55) - (3.58) we have used the values of the quark charges: $Q_{t}=\frac{2}{3}$ and $Q_{b}=-\frac{1}{3}$. We have also taken $m_{b}=0$ wherever possible. The only place where $m_{b}$ enters is in the logarithmic term:

$$
\begin{aligned}
& \mathcal{L}_{b}^{\prime}=\log \left(\frac{E_{b}+p}{E_{b}-p}\right)=\log \left[\frac{\left(1-\Delta_{t}\right)^{2}}{\Delta_{b}}\right] \\
& \mathcal{L}_{t}^{\prime}=\log \left(\frac{E_{t}+p}{E_{t}-p}\right)=\log \left(\frac{1}{\Delta_{t}}\right)
\end{aligned}
$$

where $\Delta_{b}$ is the analogue of $\Delta_{t}$ for the $b$ quark: $\Delta_{b}=m_{b}^{2} / \hat{s}$.

The cross sections as displayed in Eqs. (3.55) - (3.58) are rather complicated but their general structure is easily understood. Note that it is Eqs. (3.56) and (3.57) that feature the logarithmic enhancement coming from the forward direction. The bulk of Eq. (3.56) comes from the $b$ quark exchange diagram which is 
enhanced when the $t$ is emitted in the direction of the $W^{+}$momentum and is lefthanded. In this case the virtual $b$ quark becomes almost on-shell. The logarithmic enhancement in Eq. (3.57) comes from the $t$ quark exchange diagram in exactly the same way. Note that the difference in the coefficients of $\mathcal{L}_{b}^{\prime}$ in Eq. (3.56)and $\mathcal{L}_{t}^{\prime}$ in Eq. (3.57) is a factor of $Q_{t}^{2} / Q_{b}^{2}=4$. The other two helicity configurations, Eqs. (3.55) and (3.58) do not receive the logarithmic enhancement.

Remaining are the processes involving longitudinal $W$ 's. The cross sections for the two photon helicities are:

$$
\begin{aligned}
& \sigma\left(\gamma_{\lambda=+1} W_{\lambda=0}^{+} \rightarrow t \bar{b}\right)=\frac{\pi N_{c} \alpha^{2}}{18 s_{W}^{2} \hat{s}}\left(\frac{m_{t}}{M_{W}}\right)^{2}\left\{8 \mathcal{L}_{t}^{\prime}\left(1+\Delta_{t}-2 \Delta_{t}^{2}-3 \Delta_{t} X_{W}\right)\right. \\
& \left.+2 \mathcal{L}_{b}^{\prime}\left(1-\Delta_{t}\right)^{2}+\left(1-\Delta_{t}\right)\left[-28\left(1-\Delta_{t}\right)-6 X_{W}\left(1+3 \Delta_{t}\right)+3 X_{W}^{2}\left(\frac{2}{\Delta_{t}}-1-\Delta_{t}\right)\right]\right\} \\
& \sigma\left(\gamma_{\lambda=-1} W_{\lambda=0}^{+} \rightarrow t \bar{b}\right)=\frac{\pi N_{c} \alpha^{2}}{18 s_{W}^{2} \hat{s}}\left(\frac{m_{t}}{M_{W}}\right)^{2}\left\{8 \Delta_{t} \mathcal{L}_{t}^{\prime}\left[3 X_{W}\left(1+\Delta_{t}\right)-3 \Delta_{t}-2 \Delta_{t}^{2}\right]\right. \\
& \left.+2 \Delta_{t}^{2} \mathcal{L}_{b}^{\prime}+\left(1-\Delta_{t}\right)\left[10+\Delta_{t}\left(28-18 X_{W}-3 X_{W}^{2}\right)-42 X_{W}-3 X_{W}^{2}+\frac{6 X_{W}^{2}}{\Delta_{t}}\right]\right\}
\end{aligned}
$$

We see that only Eq. (3.60) contains the logarithmic enhancements $\mathcal{L}_{t}^{\prime}$ and $\mathcal{L}_{b}^{\prime}$, indicating that this cross section is peaked in both the forward and backward directions. The analogous logarithmic terms in Eq. (3.61) have been omitted, since they are suppressed by powers of $\left(m_{b} / M_{W}\right)$.

To obtain the contribution to $e^{+} e^{-} \rightarrow e^{-} \bar{\nu} t \bar{b}$ from $\gamma W$ fusion we average the above cross sections over photon polarizations and integrate over the momentum fractions of the $W$ and photon. The results are plotted in Figure 3.29.

As mentioned earlier, we have no guarantee that the effective- $W$ approximation will give accurate results for $\gamma W$ fusion, even at energies as high as $2 \mathrm{TeV}$. In what 


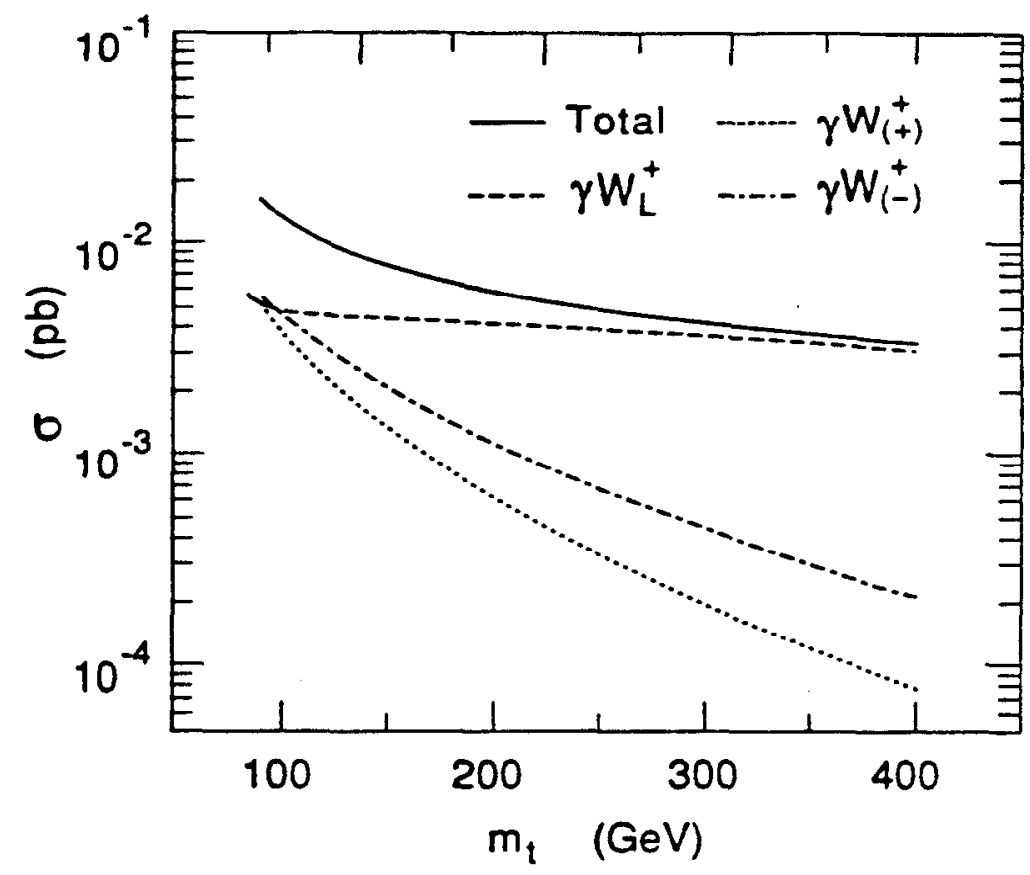

Figure 3.29 The contributions to the cross section for $e^{+} e^{-} \rightarrow e^{-} \bar{\nu} t \bar{b}$ at $\sqrt{s}=2 \mathrm{TeV}$ from $\gamma W$ fusion in the effective-boson approximation as a function of $m_{t}$ with $m_{b}=5 \mathrm{GeV}$.

follows we will check the effective- $W$ approximation by doing an exact calculation of the process $e^{+} \gamma \rightarrow \bar{\nu} t \bar{b}$. In calculating the full process $e^{+} e^{-} \rightarrow e^{-} \bar{\nu} t \bar{b}$ we will continue to treat the photon in the effective-photon approximation, since we have confidence in its accuracy. We will also neglect the peripheral diagrams that do not contribute in the effective-photon approximation.

The cross section for the process $e^{+} \gamma \rightarrow \bar{\nu} t \bar{b}$ is calculated via the diagrams in Figure 3.30. Note that the diagram coupling the electron line directly to the photon line was neglected in our effective- $W$ calculation; this diagram must be included where the effective-- $W$ approximation is relaxed, in order to cancel gauge dependent terms. The calculation of the cross section is quite lengthy. We have used REDUCE to perform the trace over $\gamma$-matrices and the angular integration of the $t \bar{b}$ system. The remaining phase-space integrals are performed numerically. 

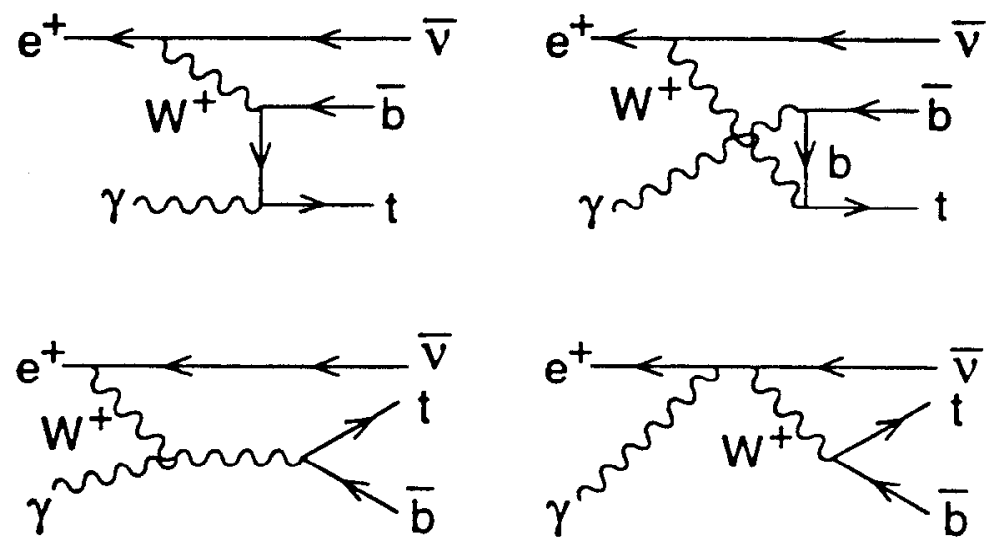

Figure 3.30 Feynman diagrams for the process $e^{+} \gamma \rightarrow \bar{\nu} t \bar{b}$.

To obtain the full cross section for $e^{+} e^{-} \rightarrow e^{-} \bar{\nu} t \bar{b}$ we integrate over the photon distribution in the electron.

To compare the exact result with that obtained from the effective- $W$ approximation we plot the differential cross section versus the energy fraction of the intermediate $W$ :

$$
\frac{d \sigma}{d x}\left(e^{+} \gamma \rightarrow \bar{\nu} t \bar{b}\right) \simeq \sum_{\lambda= \pm 1,0} f_{\lambda}(x) \sigma\left(\gamma W_{\lambda}^{+} \rightarrow t \bar{b}\right)
$$

where $x$ is the energy fraction of the $W$. This comparison is made, for several values of the top mass, in Figure 3.31. We see that we achieve very good agreement for all three masses, except at very small values of $x$ where the exact cross section increases dramatically. Figure 3.32 shows the results of integrating the exact differential cross section over the photon distribution. It agrees with the effective- $W$ calculation to within approximately $30 \%$. This process competes with the photon-photon process for top quark masses larger than $200 \mathrm{GeV}$. 


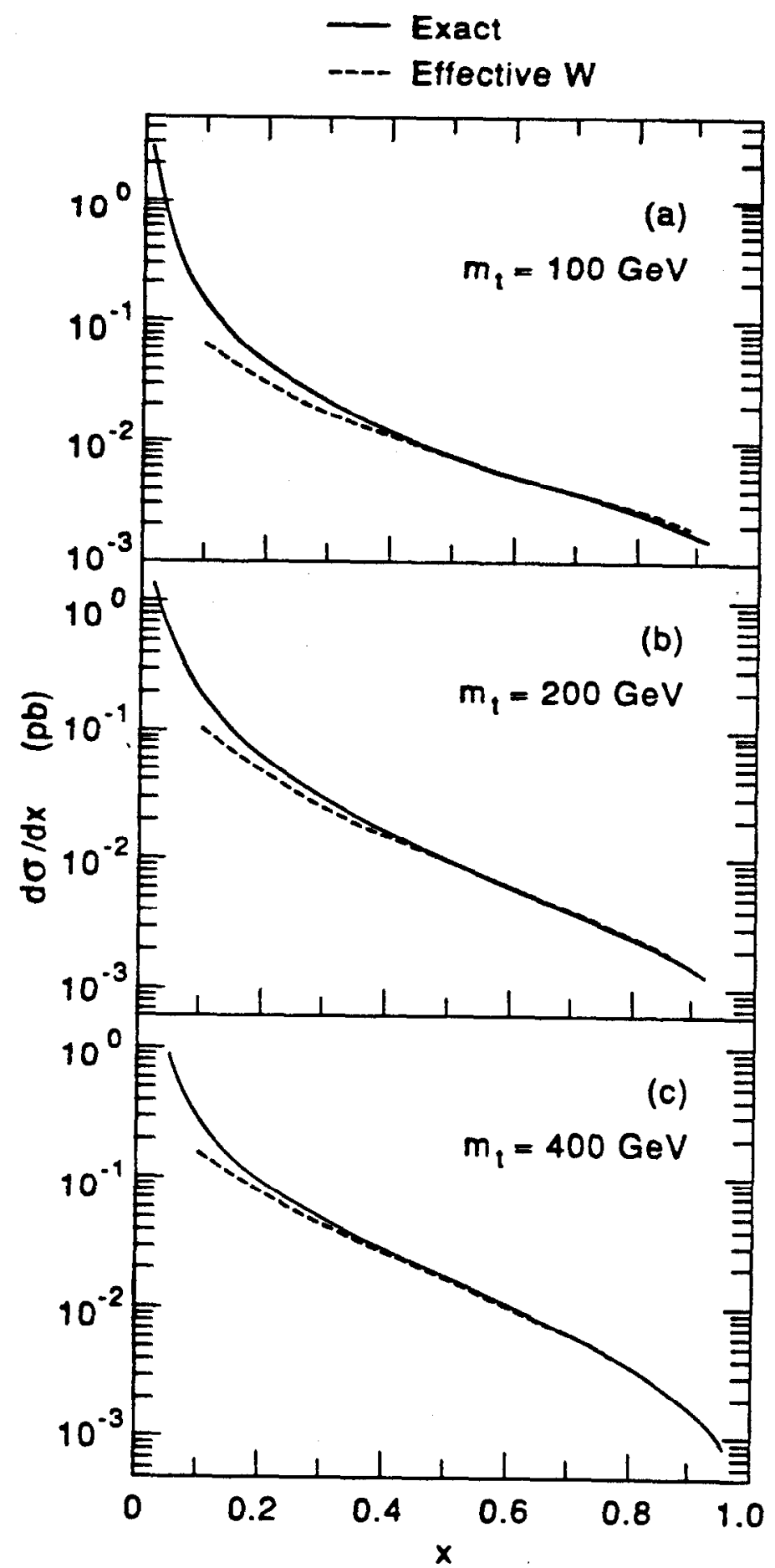

Figure 3.31 The differential cross section $d \sigma\left(e^{+} \gamma \rightarrow \bar{\nu} t \bar{b}\right) / d x$ at $\sqrt{s}=2 \mathrm{TeV}$ as a function of $\mathrm{x}$ calculated exactly compared to calculated in the effective- $W$ approximation for three choices of the top mass: a) $m_{t}=100 \mathrm{GeV}$, b) $m_{t}=200 \mathrm{GeV}$, c) $m_{t}=400 \mathrm{GeV}$. The bottom mass is $m_{b}=5 \mathrm{GeV}$. 


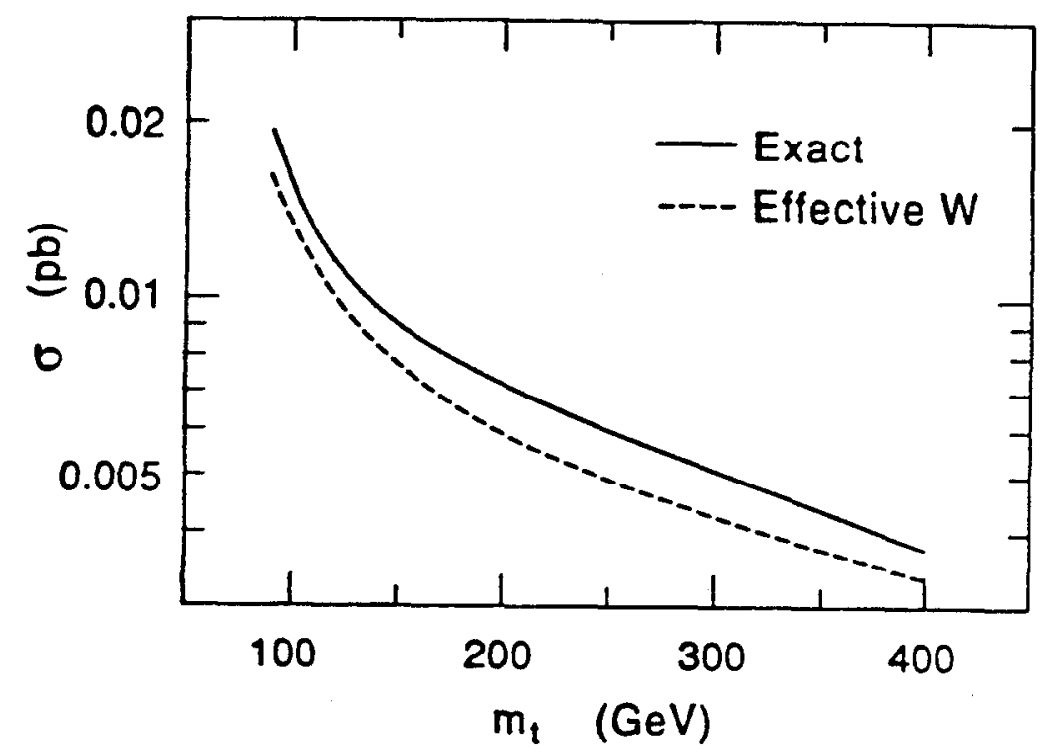

Figure 3.32 The contribution to the cross section for $e^{+} e^{-} \rightarrow e^{-} \bar{\nu} t \bar{b}$ at $\sqrt{s}=2 \mathrm{TeV}$ from $\gamma W$ fusion as a function of the top mass calculated using the effective-boson approximation for both the photon and the $W$ compared to the result using the effective-photon approximation alone and treating the $W$ exactly. The bottom mass is $m_{b}=5 \mathrm{GeV}$.

\subsection{Production of $t \bar{b}$ Pairs by $W Z$ Fusion}

The final(!) production process which we will discuss is $W Z^{0}$ fusion. This process is similar in structure to $\gamma W$ fusion, with the $Z^{0}$ taking the place of the photon. We do not expect the effective-boson approximation to have high accuracy for this process since, even for heavy top quarks, there are portions of the phase space in which the intermediate $W$ and $Z^{0}$ carry energies less than their masses. These uncertainties aside, we will use the effective-boson approximation to estimate the contribution from $W Z^{0}$ fusion.

The process $W^{+} Z^{0} \rightarrow t \bar{b}$ is calculated via the diagrams in Figure 3.33. This is the same set of diagrams we studied in the case of $W^{+} \gamma$ fusion, with the $Z^{0}$ taking the place of the photon. It is a tedious but straightforward exercise to evaluate the diagrams, and sum them to find the complete amplitude. The cross sections 


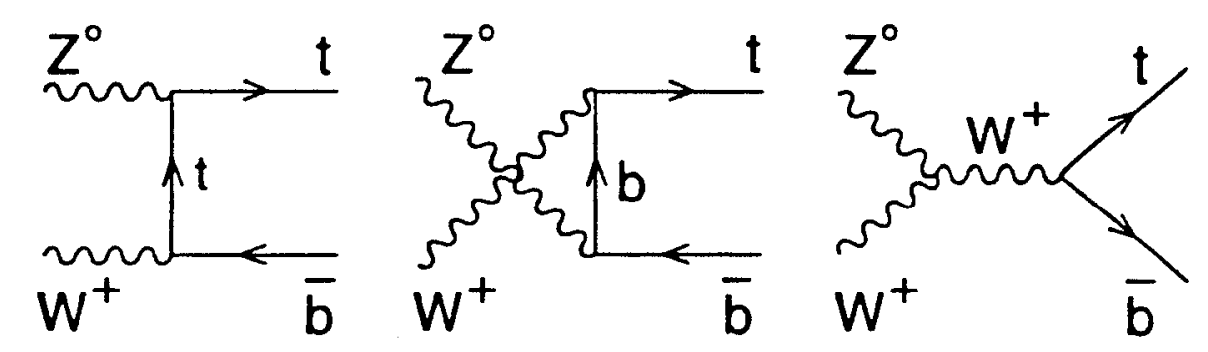

Figure 3.33 Feynman diagrams for the process $Z^{0} W^{+} \rightarrow t \bar{b}$.

for the various polarizations of the $W^{+}$and $Z^{0}$ are then obtained by squaring the amplitude and summing over the quark spins. We first write the results for the cases in which both $W^{+}$and $Z^{0}$ are transverse:

$$
\begin{aligned}
& \sigma\left(W_{\lambda=+1}^{+} Z_{\lambda=+1}^{0} \rightarrow t \bar{b}\right)=\frac{\pi N_{c} \alpha^{2}}{18 s_{W}^{4} c_{W}^{2} \hat{s}} \\
& \times\left\{\Delta_{i} \mathcal{L}_{t}^{\prime}\left[24 s_{w}^{2}\left(X_{W}^{\prime}-s_{w}^{2}\right)+\Delta_{t}\left(9-18 X_{W}^{\prime}-42 s_{w}^{2}+24 s_{w}^{2}\left(X_{w}^{\prime}+s_{w}^{2}\right)\right)\right]\right. \\
& +\left(1-\Delta_{t}\right)\left[16 s_{W}^{4}+6 X_{w}^{\prime 2}+\Delta_{t}\left(24 s_{W}^{2}-7 s_{W}^{4}+18 X_{W}^{\prime}-48 s_{w}^{2} X_{w}^{\prime}\right)\right. \\
& \left.\left.-3 \Delta_{t}^{2}\left(3 c_{w}^{4}+X_{w}^{\prime 2}\right)\right]\right\} \\
& \sigma\left(W_{\lambda=+1}^{+} Z_{\lambda=-1}^{0} \rightarrow t \bar{b}\right)=\frac{\pi N_{c} \alpha^{2}}{18 s_{W}^{4} c_{W}^{2} \hat{s}} \\
& \times\left\{\mathcal{L}_{t}^{\prime}\left[9-24 s_{w}^{2}+16 s_{W}^{4}+\Delta_{t}\left(54-132 s_{W}^{2}+80 s_{W}^{4}\right)+12 \Delta_{t}^{2} c_{W}^{2}\left(3-4 s_{W}^{2}\right)\right]\right. \\
& \left.+\left(1-\Delta_{t}\right)\left[-30+78 s_{W}^{2}-50 s_{w}^{4}-\Delta_{t}\left(57-138 s_{w}^{2}+82 s_{w}^{4}\right)-12 c_{w}^{4} \Delta_{t}^{2}\right]\right\}, \\
& \sigma\left(W_{\lambda=-1}^{+} Z_{\lambda=+1}^{0} \rightarrow t \bar{b}\right)=\frac{\pi N_{c} \alpha^{2}}{18 s_{w}^{4} c_{w}^{2} \hat{s}} \\
& \times\left\{\left(1-\Delta_{t}\right)^{2} \mathcal{L}_{b}^{\prime}\left(9-12 s_{w}^{2}+4 s_{w}^{4}\right)+\Delta_{t}^{2} \mathcal{L}_{t}^{\prime}\left(9-24 s_{w}^{2}+16 s_{w}^{4}\right)\right. \\
& \left.\left(1-\Delta_{t}\right)\left[-30+42 s_{w}^{2}-14 s_{W}^{4}+\Delta_{t}\left(33-42 s_{w}^{2}+10 s_{w}^{4}\right)-12 c_{w}^{4} \Delta_{t}^{2}\right]\right\},
\end{aligned}
$$




$$
\begin{aligned}
& \sigma\left(W_{\lambda=-1}^{+} Z_{\lambda=-1}^{0} \rightarrow t \bar{b}\right)=\frac{\pi N_{c} \alpha^{2}}{18 s_{W}^{4} c_{w}^{2} \hat{s}} \\
& \quad \times\left\{\Delta_{t}^{2} \mathcal{L}_{b}^{\prime}\left(9-12 s_{W}^{2}+4 s_{W}^{4}\right)-8 s_{W}^{2} \Delta_{t}^{2} \mathcal{L}_{t}^{\prime}\left(s_{W}^{2}-3 X_{W}^{\prime}\right)\right. \\
& \quad+\left(1-\Delta_{t}\right)\left[4 s_{W}^{4}+6 X_{W}^{\prime 2}-\Delta_{t}\left(12 s_{W}^{2}\left(1+X_{W}^{\prime}\right)-13 s_{W}^{4}+18 X_{W}^{\prime}+3 X_{W}^{\prime 2}\right)\right. \\
& \left.\left.\quad-3 \Delta_{t}^{2}\left(3 c_{W}^{4}+X_{W}^{\prime 2}\right)\right]\right\}
\end{aligned}
$$

with $X_{W}^{\prime}=c_{W}^{2} X_{W}$ and the other quantities are as defined in the previous sections. The structure of Eqs. (3.62)-(3.65) is determined by the same dynamics we discovered in our earlier studies: the coupling of the $W$ to fermions is left-handed and the spin of the fermion prefers to align with the boson to which it couples. When both of these conditions are satisfied the cross section is enhanced. The cross sections involving longitudinal $W^{\prime}$ 's and $Z^{0}$ 's are calculated in the same manner. The results for the various combinations of helicities are

$$
\begin{aligned}
& \sigma\left(W_{\lambda=-1}^{+} Z_{\lambda=0}^{0} \rightarrow t \bar{b}\right)=\frac{\pi N_{c} \alpha^{2}}{6 s_{W}^{4} c_{W}^{2}}\left(\frac{X_{W}^{\prime}}{M_{z}}\right)^{2}\left(1-\Delta_{t}\right)^{2}\left(2+\Delta_{t}\right) \\
& \sigma\left(W_{\lambda=+1}^{+} Z_{\lambda=0}^{0} \rightarrow t \bar{b}\right)=\frac{\pi N_{c} \alpha^{2}}{12 s_{W}^{4} c_{W}^{2} \hat{s}}\left(\frac{m_{t}}{M_{z}}\right)^{2} \\
& \times\left\{3\left(1+4 X_{W} c_{W}^{2}\right)\left(\mathcal{L}_{t}^{\prime}-1+\Delta_{t}\right)+\frac{2 c_{W}^{4} X_{W}^{2}}{\Delta_{t}}\left(1-\Delta_{t}\right)^{2}\left(2+\Delta_{t}\right)\right\} \\
& \sigma\left(W_{\lambda=0}^{+} Z_{\lambda=+1}^{0} \rightarrow t \bar{b}\right)=\frac{\pi N_{c} \alpha^{2}}{36 s_{W}^{4} c_{W}^{2} \hat{s}}\left(\frac{m_{t}}{M_{W}}\right)^{2}\left\{\left(9-12 s_{W}^{2}+4 s_{W}^{4}\right)\left(1-\Delta_{t}\right)^{2} \mathcal{L}_{b}^{\prime}\right. \\
& +\mathcal{L}_{t}^{\prime}\left[16 s_{W}^{4}\left(1+\Delta_{t}-2 \Delta_{t}^{2}\right)-48 \Delta_{t} s_{W}^{2} X_{W}^{\prime}-27 \Delta_{t}^{2}\right] \\
& \left(1-\Delta_{t}\right)\left[-18+48 s_{W}^{2}-56 s_{W}^{4}+12 X_{W}^{\prime}\left(3+s_{W}^{2}\right)-6 X_{W}^{\prime 2}+12 \frac{X_{W}^{\prime 2}}{\Delta_{t}}\right. \\
& \left.\left.+\Delta_{t}\left(45-108 s_{W}^{2}+56 s_{W}^{4}-36 c_{W}^{2} X_{W}^{\prime}-6 X_{W}^{\prime 2}\right)\right]\right\}
\end{aligned}
$$




$$
\begin{aligned}
\sigma\left(W_{\lambda=0}^{+}\right. & \left.Z_{\lambda=-1}^{0} \rightarrow t \bar{b}\right)=\frac{\pi N_{c} \alpha^{2}}{36 s_{W}^{4} c_{W}^{2} \hat{s}}\left(\frac{m_{t}}{M_{W}}\right)^{2} \\
& \times\left\{\mathcal { L } _ { t } ^ { \prime } \left[36 X_{W}^{\prime}-48 s_{W}^{2} X_{W}^{\prime}-12 \Delta_{t}\left(3-7 s_{W}^{2}+4 s_{W}^{4}-3 X_{W}^{\prime}+4 s_{W}^{2} X_{W}^{\prime}\right)\right.\right. \\
& \left.-\Delta_{t}^{2}\left(27-60 s_{W}^{2}+32 s_{W}^{4}\right)\right]+\Delta_{t}^{2} \mathcal{L}_{b}^{\prime}\left(9-12 s_{W}^{2}+4 s_{W}^{4}\right) \\
& +\left(1-\Delta_{t}\right)\left[9-24 s_{W}^{2}+20 s_{W}^{4}-12 X_{W}^{\prime}\left(6-7 s_{W}^{2}\right)-6 X_{W}^{\prime 2}\right. \\
& \left.\left.+\Delta_{t}\left(45-108 s_{W}^{2}+56 s_{W}^{4}-36 c_{W}^{2} X_{W}^{\prime}-6 X_{W}^{\prime 2}\right)+\frac{12 X_{W}^{\prime 2}}{\Delta_{t}}\right]\right\}
\end{aligned}
$$

The leading terms in Eqs. (3.66)-(3.69) are proportional to $\left(m_{t} / M_{z}\right)^{2}$ or $\left(m_{t} / M_{W}\right)^{2}$, as expected.

The calculation of the fusion of a longitudinal $W^{+}$and a longitudinal $Z^{0}$ is slightly more subtle than the previous cases. Our general program has been to use light-like momenta for the $W^{+}$and $Z^{0}$ and to use longitudinal polarization vectors, $\epsilon^{\mu}=k^{\mu} / M_{V}$. The cancellations involved in the full longitudinal calculation are very delicate. If the bosons are not taken on their physical mass shell the cancellations do not take place. Of course, if the physical momenta and polarization vectors are used the cancellations occur and the unitary behavior of the gauge theory is seen. Our procedure will be to calculate the full amplitude for on-shell bosons. This amplitude will be free of unitarity-violating behavior. We will then continue the amplitude back to $k^{2}=0$ and $\epsilon^{\mu}=k^{\mu} / M_{v}$ for both $W^{+}$and $Z^{0}$.

The result of this procedure is 


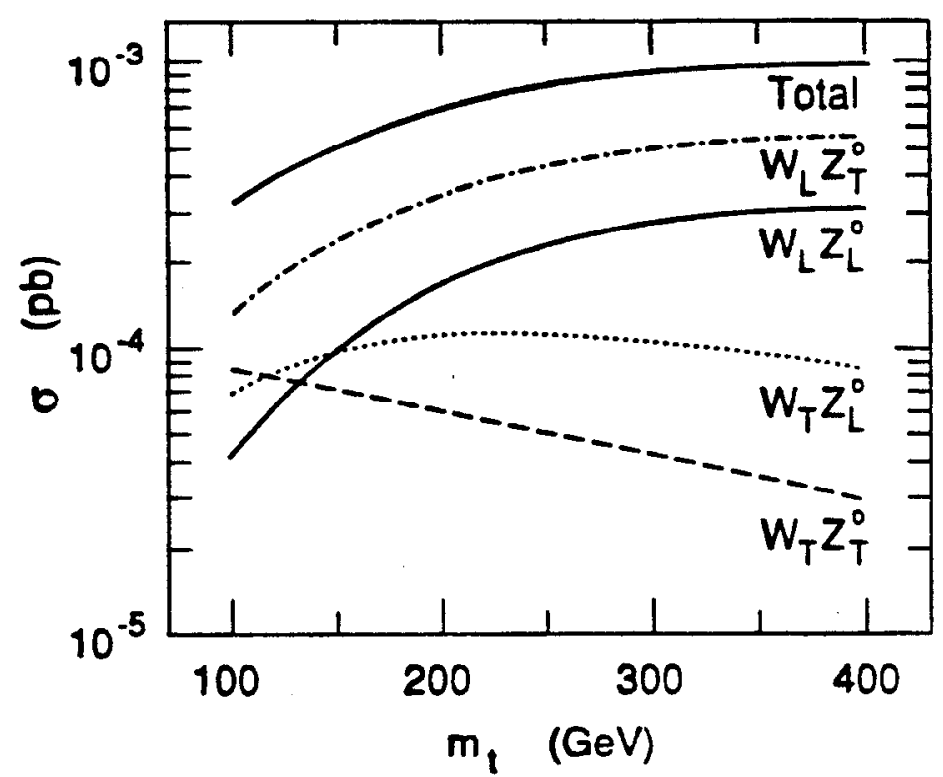

Figure 3.34 Contributions to the cross section for $t \bar{b}$ production at $\sqrt{s}=2 \mathrm{TeV}$ via $W Z$ fusion in the effective-boson approximation as a function of the top mass with $m_{b}=5 \mathrm{GeV}$.

$$
\begin{aligned}
\sigma\left(W_{\lambda=0}^{+}\right. & \left.Z_{\lambda=0}^{0} \rightarrow t \bar{b}\right)=\frac{\pi N_{c} \alpha^{2}}{24 s_{W}^{4} c_{W}^{2} \hat{s}}\left(\frac{m_{t}^{4}}{M_{w}^{2} M_{z}^{2}}\right) \\
& \times\left\{3 \mathcal{L}_{t}^{\prime}\left(1-4 X_{W}^{\prime}+2 X_{W}\right)+\left(1-\Delta_{t}\right)\left[-3-6 s_{W}^{2} X_{W}-\left(1-2 s_{W}^{2}+4 s_{W}^{4}\right) X_{W}^{2}\right.\right. \\
& \left.\left.+\frac{1}{\Delta_{t}}\left(6 X_{W}^{\prime}+\left(-1+2 s_{W}^{2}+2 s_{w}^{4}\right) X_{W}^{2}\right)+\frac{2 X_{W}^{\prime 2}}{\Delta_{t}^{2}}\right]\right\}
\end{aligned}
$$

The cross section has the expected factor of $m_{t}^{4}$.

To find the contribution to $t \bar{b}$ pair production from these processes we need to repeat the familiar process of folding in the effective boson distributions and performing the integrals over the momentum fractions. The results of this are shown in Figure 3.34, as a function of $m_{t}$. The results are comparable with those from $W W$ fusion. 


\subsection{BEAMSTRAHLUNG}

Since most processes of interest in a linear collider scale like $1 / s$ it is necessary for a $\mathrm{TeV}$ linear collider to have a very high luminosity. If the process of interest has a cross section on the order of $\sigma_{\mathrm{pt}}$ then a luminosity of order $10^{33} \mathrm{~cm}^{-2} \mathrm{sec}^{-1}$ is necessary to produce $10^{3}$ events in a year. At the bunch densities required to produce this luminosity the bulk interactions between the bunches become important. One of the consequences is that the incoming electrons bremsstrahlung in the field of the positron bunch. ${ }^{30}$ This intense radiation, called "beamstrahlung," smears out the energy of the electrons and positrons.

There is also the possibility of the beamstrahlung photons interacting with each other and the electrons and positrons in the colliding beams. Blankenbecler and Drell $^{31}$ have shown that the effective luminosities for photon-photon or photonelectron collisions can be quite large, depending on the parameters of the machine. The beamstrahlung two-photon luminosity can even dominate the flux of "virtual" photons, calculated in the effective-photon approximation, as shown in Figure 3.35. The electron-photon luminosity, plotted in Figure 3.36, is also substantial.

We can easily calculate the production of top quarks from the fusion of beamstrahlung photons:

$$
\sigma_{\text {beamstrahlung }}\left(e^{+} e^{-} \rightarrow e^{+} e^{-} t \bar{t}\right)=\left.\int d z \frac{d \mathcal{L}_{\gamma \gamma}}{d z} \sigma(\gamma \gamma \rightarrow t \bar{t})\right|_{\sqrt{\hat{s}=z \sqrt{s}}} \cdot
$$

The results of the numerical integration are shown Figure 3.37. We see that for our chosen set of beam parameters the beamstrahlung production process dominates the effective-photon process by two orders of magnitude. 


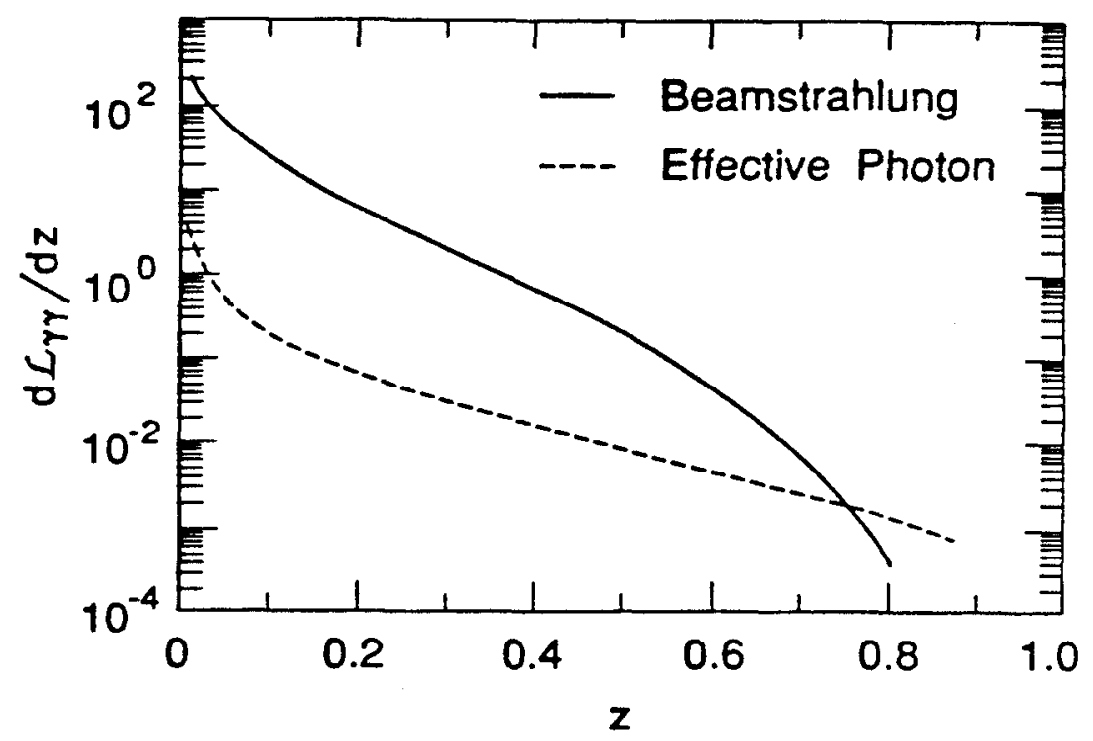

Figure 3.35 The differential photon-photon luminosity $d \mathcal{L}_{\gamma \gamma} / d z$ relative to the incident electron-positron flux as a function of the energy fraction $z=\sqrt{\hat{s} / s}$ at a $2 \mathrm{TeV}$ linear collider. The bunches are taken to have circular cross section and the luminosity and laboratory bunch length are: $\mathcal{L} \sim 2.8 \times 10^{30} \mathrm{~cm}^{-2}$ and $l_{0} \sim 0.15 \mathrm{~mm}$, respectively. The two-photon flux from the effective-photon approximation is shown for comparison. (Reprinted with permission from Blankenbecler and Drell, Ref. 31.)

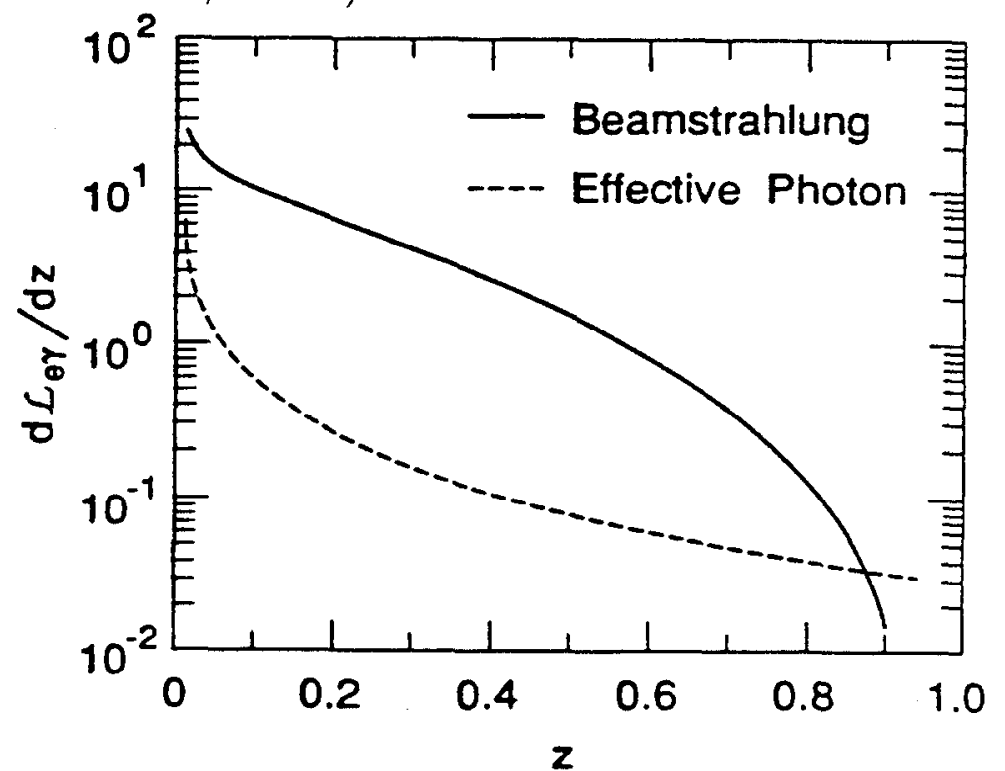

Figure 3.36 The differential photon-electron luminosity $d \mathcal{L}_{e \gamma} / d z$ relative to the incident electron-positron flux as a function of the energy fraction $z=\sqrt{\hat{s} / s}$. The beam parameters are the same as those in Figure 3.35. The photon flux from the effective-photon approximation is shown for comparison. (Reprinted with permission from Blankenbecler and Drell, Ref. 31.) 


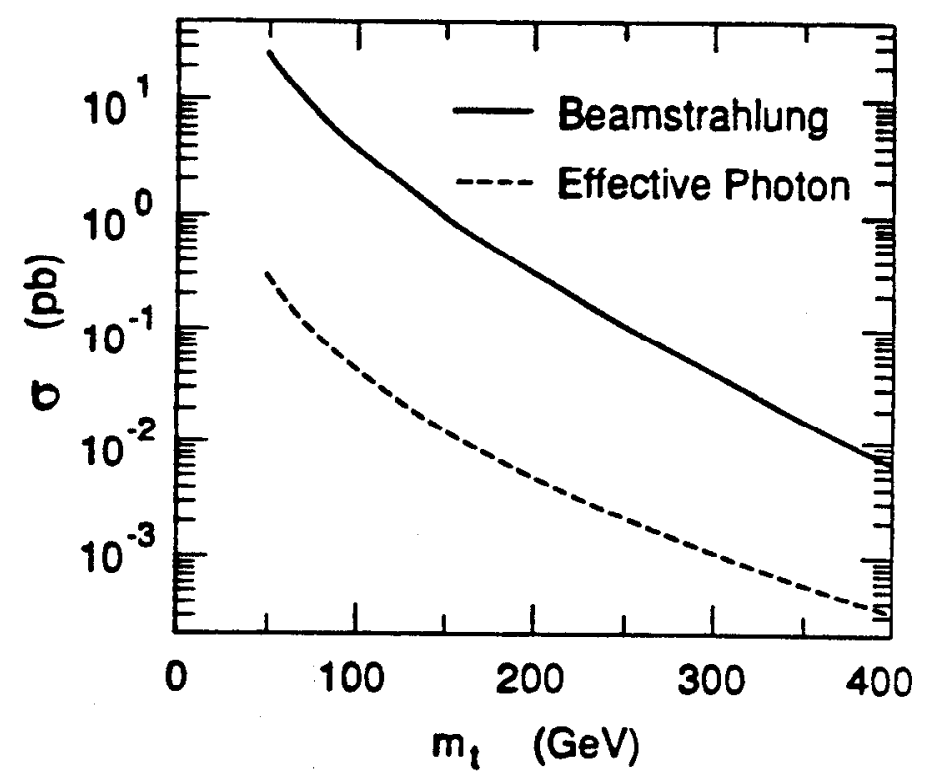

Figure 3.37 The cross section for production of top quarks by fusion of beamstrahlung photons as a function of the top quark mass for the same choice of beam parameters as in Figure 3.35. The contribution from the effective-photon approximation is displayed for comparison.

We can also calculate the production of $t-\bar{b}$ pairs as a result of the interaction of beamstrahlung photons interacting with positrons:

$$
\sigma_{\text {beamstrahlung }}\left(e^{+} e^{-} \rightarrow \bar{\nu} e^{-} t \bar{b}\right)=\left.\int d z \frac{d \mathcal{L}_{\text {eq }}}{d z} \sigma\left(e^{+} \gamma \rightarrow \bar{\nu} t \bar{b}\right)\right|_{\sqrt{\hat{s}}=z \sqrt{s}} .
$$

This cross section, calculated in the effective- $W$ approximation, is displayed in Figure 3.38. Again the beamstrahlung contribution dominates. Of course an equal number of anti-top quarks are produced in the charge-conjugate reaction. These results were calculated for the case of circular beams which give the maximum beamstrahlung flux. For flat beams the fluxes can be reduced by an order of magnitude. 


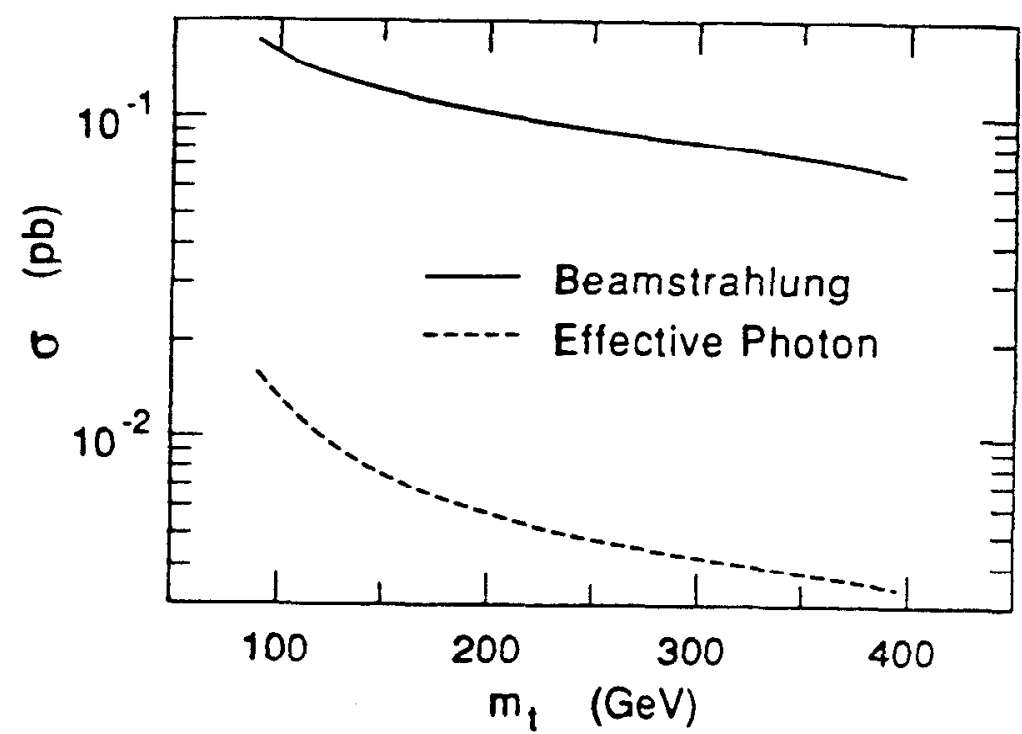

Figure 3.38 The cross section for production of $t-\bar{b}$ pairs by fusion of beamstrahlung photons with virtual $W$ bosons, calculated in the effective- $W$ approximation, as a function of the top quark mass for the same choice of beam parameters as in Figure 3.35. The contribution from the effective-photon approximation is displayed for comparison.

\subsection{SUMMARY AND CONCLUSIONS}

After reviewing the range of possible vector boson fusion processes we see that the $\gamma \gamma$ fusion dominates at the smaller top masses, $m_{t}<200 \mathrm{GeV}$ and exceeds lowest order cross section for $m_{t}<100 \mathrm{GeV}$. At larger masses, fusion of longitudinal $W$ 's exceeds the $\gamma \gamma$ result, due to the enhanced $W$ couplings. Processes involving $Z^{0}$ 's are seen to be an order of magnitude smaller than the analogous processes involving $W$ 's. The interference between photons and $Z^{0}$ 's is seen to be much smaller than one would naively expect due to the small vector coupling of the electron to the $Z^{0}$. Our check of the effective- $W$ approximation in the reaction $e+\gamma \rightarrow \bar{\nu} t \bar{b}$ shows that it is accurate to within $30 \%$. Including beamstrahlung can greatly enhance the photon fluxes. For circular beams the photon-photon cross section dominates both the lowest order cross section and the effective-photon 
result for $m_{t}<200 \mathrm{GeV}$.

These results are easily transferable to production of other heavy quarks or heavy leptons. In $\gamma \gamma$ fusion we simply need to scale by the charge to the fourth power. The leading terms in processes involving longitudinal $W$ and $Z^{0}$ s are the same for up-type quarks, down-type quarks and heavy leptons.

Our results on processes involving longitudinal $W$ 's and $Z^{0}$ 's agree with those presented by Yuan, Ref. 23 and Eboli et al., Ref. 23. 


\section{REFERENCES}

1. S. L. Wu, in Proceedings of the 1987 International Symposium on Lepton and Photon Interactions at High Energies, Hamburg, July 27-31, edited by W. Bartel and R. Ruckl, (North Holland, Amsterdam, 1988), p.39.

2. G. L. Kane and M. E. Peskin, Nucl. Phys. B195, 29 (1982).

3. A. Bean et al., Phys. Rev. D35, 3533 (1987); W. Bartel et al., Phys. Lett. 132B, 241 (1983).

4. S. Uno, invited talk at the Seventeenth SLAC Summer Institute on Particle Physics, Stanford, July 10-21, 1988 (unpublished).

5. I. Wingerter, in International Symposium on Production and Decay of Heavy Flavors, Stanford, September 1-5, 1987, edited by E. Bloom and A. Fridman, (New York Academy of Sciences, New York, 1988), vol 535, p. 1.

6. J. Ellis, J. S. Hagelin and S. Rudaz, Phys. Lett. 192B, 201 (1987); I. I. Y. Bigi and A. I. Sanda, Phys. Lett. 194B, 307 (1987); F. J. Gilman, in The Fourth Family of Quarks and Leptons, edited by D. B. Cline and A. Soni, (The New York Academy of Sciences, New York, 1987), p.159; G. Altarelli and P. J. Franzini, Z. Phys. C37, 271 (1988); H. Harari and Y. Nir, Phys. Lett. 195B, 586 (1987); V. A. Khoze and N. G. Uraltsev, Leningrad preprint, 87-1290, 1987 (unpublished); L. L. Chau and W. Y. Keung, UC Davis preprint UCD-87-02, 1987 (unpublished); J. F. Donoghue et al., Phys. Lett. 195B, 285 (1987); A. Ali, in UCLA Collider Workshop, 1987, p. 110; J. R. Cudell et al., Phys. Lett. 196B, 227 (1987); A. Datta, E. A. Paschos and U. Turke, Phys. Lett. 196B, 376 (1987); D. Du and 
Z. Zhao, Phys. Rev. Lett. $\underline{59}, 1072$ (1987). See also F. Halzen, C. S. Kim and S. Pakvasa, University of Wisconsin preprint MAD/PH/394, 1987 (unpublished).

7. H. Albrecht et al., Phys. Lett. 192B, 245 (1987).

8. T. Rogdrigo, invited talk at the Seventeenth SLAC Summer Institute on Particle Physics, Stanford, July 10-21, 1989 (unpublished); T. Pal, it ibid (unpublished); K. Ragan, ibid (unpublished).

9. Most of the content of Chapter 2 has been pubished in F. J. Gilman and R. Kauffman, Phys. Rev. D37, 2676 (1988).

10. J. H. Kühn, Acta Phys. Polonica B12, 347 (1981); V. Barger, H. Baer and K. Hagiwara, Phys. Rev. D30, 947 (1984); I. I. Y. Bigi et al., Phys. Lett. 181B, 157 (1986); S. Geer, G. Pancheri and Y. N. Srivastava, Phys. Lett. 192B, 223 (1987). See also A. Martin, in Heavy Flavors: Proceedings, May 19-25, 1987, San Miniato, Italy, edited by F. L. Navarria, (North-Holland, Amsterdam, 1988), p. 133; and M. Jeżabek and J. H. Kühn, Phys. Lett. 207B, 91 (1988).

11. Particle Data Group, Phys. Lett. 204B, 1 (1988).

12. We take the total width of the $W$ to be $\Gamma_{W}=2.25 \mathrm{GeV}=9 \Gamma\left(W \rightarrow e \nu_{e}\right)$, accounting for three generations of leptons and only two of quarks, since here we are only interested in the situation where $W^{+} \rightarrow t \bar{b}$ is kinematically forbidden or at least very highly suppressed.

13. I. I. Y. Bigi and H. Krasemann, Z. Phys. $\underline{\text { C7, }} 127$ (1981).

14. I. Hinchliffe and C. H. Llewellyn Smith, Nucl. Phys. B114, 45 (1976);

I. Hinchliffe and C. H. Llewellyn Smith, Phys. Lett. $\underline{61 B}, 472(1976)$; 
15. B. Grinstein, N. Isgur and M. B. Wise, Phys. Rev. Lett. 56, 298 (1986); B. Grinstein, N. Isgur and M. B. Wise, California Institute of TechnologyUniversity of Toronto Report No. CALT-68-1311, UTPT-85-37, 1985 (unpublished); B. Grinstein and M. B. Wise, California Institute of Technology Report No. CALT-68-1445, 1987 (unpublished); B. Grinstein, N. Isgur, D. Scora and M. B. Wise, Phys. Rev. D39, 779 (1989).

16. T. Altomari and L. Wolfenstein, Phys. Rev. D37, 681 (1988) and Phys. Rev. Lett. 58, 1583 ( 1987).

17. The direction of muons, taus or quark jets in other decays of the $W$ will of course serve the same purpose.

18. C. Ahn et al., SLAC Report 329, May 1988, and references therein; G. Altarelli in Proceedings of the Workshop on Physics at Future Accelerators (La Thuile), J. H. Mulvey, Ed. (CERN, Geneva, 1987); J. Ellis and F. Pauss, ibid.

19. E.J. Williams, Kgl. Danske Videnskab. Selskab, Mat-Fys. Medd. 13 (No. 4), (1934); L. Landau and E. Lifshitz, Physik Z. Sowetjetunion 6, 244 (1934).

20. E. Fermi, Z. Physik 29, 315 (1924); C. Weizsäcker and E. J. Williams, ibid, 88, 612 (1934); L. Landau and E. Lifshitz, Physik Z. Sowetjetunion 6, 244 (1934).

21. S. J. Brodsky, T. Kinoshita, H. Terazawa, Phys. Rev. D 4, 1532 (1971).

22. R. Cahn and S. Dawson, Phys. Lett. 136B, 196 (1984), Erratum ibid, p. 464; M. Chanowitz and M. K. Gaillard, Phys. Lett. 142B, 85 (1984); G. L. Kane, W. W. Repko, and W. B. Rolnick, Phys. Lett. 148B,367 
(1984); S. Dawson, Nucl. Phys. B249,42 (1985); J. Lindfors, Z. Phys.

C28, 427 (1985); J. F. Gunion, J. Kalinowski, and A. Tofighi-Niaki, Phys.

Rev. Lett. 57, 2351 (1986); W. B. Rolnick, Nucl. Phys. B274, 171 (1986);

P. W. Johnson, F. I. Olness, W.-K. Tung, Phys. Rev. D36, 291 (1987).

Z. Kunszt, Nucl. Phys. B296, 253 (1988).

23. S. Dawson, S. S. D. Willenbrock, Nucl. Phys. B284, 449 (1987); C. P. Yuan, Nucl. Phys. B310, 1 (1988); D. A. Dicus, K. J. Kallianpur, S. S. D. Willenbrock, Phys. Lett. 200B, 187 (1988); O. J. P. Eboli et al., Phys. Rev. D34, 771 (1986). O. J. P. Eboli et al., Sao Paulo University Report IFT/P-37/88, (unpublished).

24. In the original paper by Dawson, Ref. 22, it was not discovered that the longitudinal terms do not interfere with the transverse ones. That all the interference terms vanish was pointed out by Johnson, Olness, and Tung, Ref. 22, and also by Lindfors, Ref. 22 .

25. This term was omitted in the original paper by Dawson, Ref. 22 .

26. This cancellation was noted by Johnson, Olness and Tung, Ref. 21, and Lindfors, Ref. 21.

27. A. Abbasabadi, D. A. Dicus, W. W. Repko, and R. Vega, Phys. Rev. D38, 2770 (1988); D. A. Dicus, Nucl. Phys. B287, 397 (1987).

28. Appelquist and Chanowitz have used this to place a unitarity bound on the scale of electro-weak symmetry breaking: T. Appelquist and M. Chanowitz, Phys. Rev. Lett. 59, 2405 (1987), Erratum, ibid, 60, 1589 (1988). 
29. Fermion pair production via $\gamma Z^{0}$ fusion has also been studied J. Layssac, H. Leveque, and G. Moultaka, Phys. Lett. 209B, 538 (1988).

30. R. Blankenbecler and S. D. Drell, Phys. Rev. D36, 277 (1987) and 37, 3308 (1988); M. Jacob and T. T. Wu, Phys. Lett bf B197, 253 (1987), Nucl. Phys. B303, 373, (1988), and ibid, p. 389; M. Bell and J. S. Bell, Part. Accel. 22, 301 (1988) and ibid, 24, 1 (1988); V. N. Băer and V. M. Katkov, Zh. Eksp. Teor. Fiz. 55, 1542 (1968) [Sov. Phys. JETP 28, 807 (1969)] and Zh. Eksp. Teor. Fiz. 53, 1478 (1967) [Sov. Phys. JETP 26, 854 (1968)].

31. R. Blankenbecler and S. Drell, Phys. Rev. Lett. 61, 2324 (1988). 